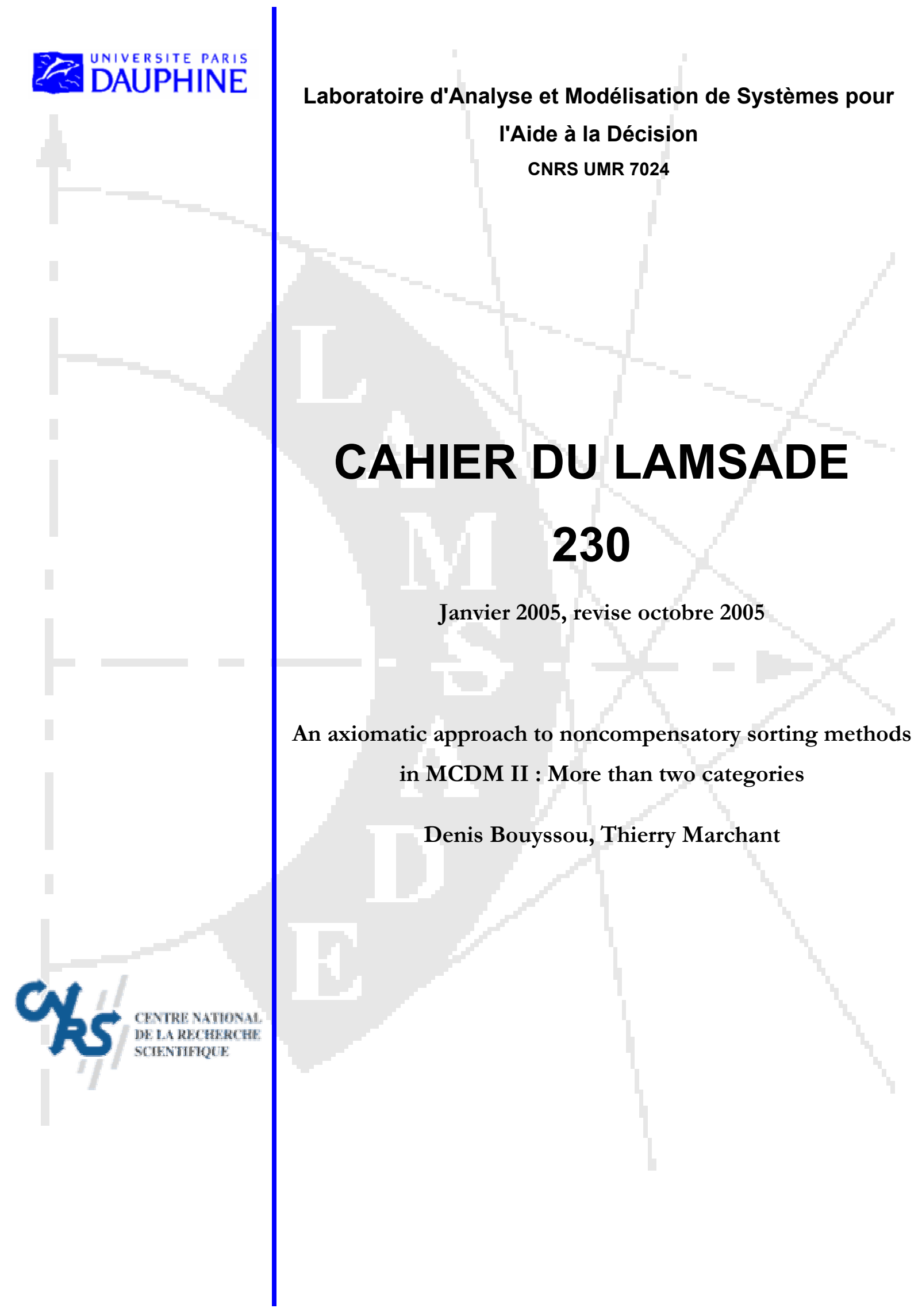




\section{An axiomatic approach to noncompensatory sorting methods in MCDM, II: More than two categories ${ }^{1}$}

\author{
Denis Bouyssou ${ }^{2}$ \\ CNRS - LAMSADE
}

\author{
Thierry Marchant ${ }^{3}$ \\ Ghent University
}

10 January 2005

Revised 25 October 2005

\footnotetext{
${ }^{1}$ We wish to thank Jose Figueira and Marc Pirlot for their comments on an earlier draft of this text. Our greatest debt is to Salvatore Greco, Benedetto Matarazzo and Roman Słowiński who alerted us on the relation between our results on noncompensatory sorting models and the results in Słowiński et al. (2002) on sorting models using a Sugeno integral. Furthermore, their detailed comments have much contributed to improve an earlier version of this text. The usual caveat clearly applies.

${ }^{2}$ LAMSADE, Université Paris Dauphine, Place du Maréchal de Lattre de Tassigny, F-75775 Paris Cedex 16, France, tel: +331440548 98, fax: +3314405 40 91, e-mail: bouyssou@lamsade.dauphine.fr, Corresponding author.

${ }^{3}$ Ghent University, Department of Data Analysis, H. Dunantlaan 1, B9000 Gent, Belgium, tel: +32 926463 73, fax: +32 926464 87, e-mail: thierry.marchant@UGent. be.
} 


\begin{abstract}
A number of recent papers have investigated the foundations of methods allowing to sort multi-attributed alternatives between several ordered categories. This paper has a similar objective. Our analysis uses a general conjoint measurement framework, encompassing most sorting models used in MDCM, that was proposed in the literature. Within this framework, we provide an axiomatic analysis of what we call noncompensatory sorting models, with or without veto effects. These noncompensatory sorting models contain the pessimistic version of ELECTRE TRI as a particular case. Our analysis can be seen as an attempt to give a firm axiomatic basis to ELECTRE TRI, while emphasizing its specific feature, i.e., the rather poor information that this model uses on each attribute.
\end{abstract}

Keywords: Decision with multiple attributes, Sorting models, Noncompensation, Conjoint measurement, ELECTRE TRI. 


\section{Contents}

1 Introduction and motivation 1

2 Definitions and notation $\quad 2$

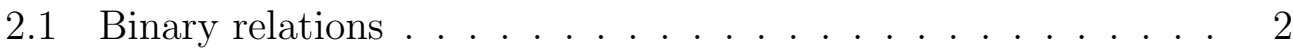

2.2 The setting . . . . . . . . . . . . . . . 3

2.3 Primitives ..................... . . . . . . 3

3 A general measurement framework 4

3.1 The model . . . . . . . . . . . . . . . . . . . 4

3.2 Interpretations of model $(D 1) \ldots \ldots . \ldots$

4 ELECTRE TRI 13

5 The noncompensatory sorting model 16

5.1 Definitions . . . . . . . . . . . . . . 16

5.2 Axioms . . . . . . . . . . . . . . . . . 18

5.3 Background on twofold partitions . . . . . . . . . . . 21

5.4 Result . . . . . . . . . . . . . . . . . 22

5.5 The noncompensatory sorting model and the Sugeno integral . 25

5.6 Independence of axioms . . . . . . . . . . . . . . . . . . . . 28

5.7 Uniqueness of the representation . . . . . . . . . . . . . 29

5.8 Extensions . . . . . . . . . . . . . . . . . . 32

5.8.1 The case $\mathscr{A}_{i}^{k}=\mathscr{A}_{i}^{\ell} \ldots \ldots \ldots . \ldots . \ldots 32$

5.8.2 The case $\mathscr{F}^{k}=\mathscr{F}^{\ell} \ldots \ldots \ldots . \ldots . \ldots 34$

6 The noncompensatory sorting model with veto 35

6.1 Definitions ................... 35

6.2 Axioms . . . . . . . . . . . . . . . . . 37

6.3 Result . . . . . . . . . . . . . . . . . . . 39

6.4 Independence and uniqueness . . . . . . . . . . . . . . . . 44

6.4.1 Independence of conditions . . . . . . . . . . . . . . . 45

6.4.2 Uniqueness ................. . . 45

$\begin{array}{lll}7 & \text { Conclusion } & 46\end{array}$

$\begin{array}{ll}\text { References } & 47\end{array}$ 


\section{Introduction and motivation}

In most MCDM models, a recommendation is built using a preference relation comparing alternatives in terms of desirability. Hence, in these models, the recommendation is derived on the basis of a relative evaluation model as given by the preference relation. This is not always appropriate since, e.g., the best alternatives may not be desirable at all. This calls for evaluation models having a more absolute character. In response to this need, the MCDM community has recently developed a number of techniques designed to sort alternatives between ordered categories defined by norms. Recent reviews of this trend of research can be found in Greco et al. (1999b, 2002a,b) and Zopounidis and Doumpos (2000a, 2002). Contrary to more traditional approaches based on binary relations for which conjoint measurement provides a firm theoretical basis, these techniques have often been proposed on a more or less ad hoc basis.

A recent research trend (see Greco et al., 2001b; Słowiński et al., 2002) has investigated the theoretical foundations of such methods, adapting traditional conjoint measurement techniques to deal with ordered partitions of multi-attributed alternatives. The aim of this paper is to contribute to this trend.

In a companion paper (Bouyssou and Marchant, 2005), we proposed an axiomatic analysis of several sorting models between two categories, concentrating on what we called noncompensatory sorting models. These models contain the pessimistic version of ELECTRE TRI as a particular case. As explained in Bouyssou and Marchant (2005), the choice of ELECTRE TRI was motivated by the fact that this model has generated numerous studies (see Dias and Clímaco, 2000; Dias and Mousseau, 2006; Dias et al., 2002; Lourenco and Costa, 2004; Mousseau et al., 2001a; Mousseau and Słowiński, 1998; Ngo The and Mousseau, 2002; Tervonen et al., 2005) and has often been applied in practice (see, e.g., Andenmatten, 1995; Arondel and Girardin, 2000; Georgopoulou et al., 2003; Moussa, 2001; Mousseau et al., 2000a, 2001b; Roy, 2002).

The main aim of this paper is to extend this analysis to the case of an arbitrary (finite) number of ordered categories. We refer the reader to Bouyssou and Marchant (2005) for a detailed motivation of such an analysis, its relation to the literature and its possible implications for the practice of MCDM. The present paper mostly concentrates on technical results. It can be read independently of Bouyssou and Marchant (2005). Our results will turn to have close connections with some of the results in Greco et al. (2001b) and Słowiński et al. (2002). These connections will be analyzed in detail.

This paper is organized as follows. We introduce our setting in Section 2. 
Section 3 introduces a general conjoint measurement framework that was proposed in the literature. Section 4 presents the main points of the ELECTRE TRI sorting technique. Section 5 deals with the case of noncompensatory sorting models. These models are roughly equivalent to the ELECTRE TRI method when there is no discordance effect. Section 6 extends this analysis to include the possibility of such effects. A final section concludes.

Throughout the paper, remarks contain comments that can be skipped on first reading without loss of continuity.

\section{Definitions and notation}

\subsection{Binary relations}

We use a standard vocabulary for binary relations. An equivalence (resp. a weak order; a total order; a semiorder) is a reflexive, symmetric and transitive (resp. complete and transitive; complete, antisymmetric and transitive; complete, Ferrers and semi-transitive) relation.

When $T$ is an equivalence relation on $A, A / T$ will denote the set of equivalence classes of $T$ on $A$. A partition of $A$ is a collection of nonempty subsets of $A$ that are pairwise disjoint and such that the union of the elements in this collection is $A$. It is clear that, when $T$ is an equivalence relation on $A, A / T$ is a partition of $A$. Indeed, defining a partition of $A$ is tantamount to defining an equivalence relation on $A$.

When $T$ is reflexive and transitive, its symmetric part $\iota(T)$ is an equivalence. It will prove convenient to speak of the equivalence classes of $T$ to mean the equivalence classes of its symmetric part $\iota(T)$. When $T$ is a weak order, it induces on a total order on $A / \iota(T)$. When $T$ is a weak order and $A / \iota(T)$ is finite, we will often speak of the first or last equivalence class of $T$.

Let $T$ be a weak order on $A$. Following, e.g., Krantz et al. (1971, Chapter 2), we say that $B$ is dense in $A$ for $T$ if, for all $a, b \in A,[a T b$ and $\operatorname{Not}[b T a]] \Rightarrow[a T c$ and $c T b$, for some $c \in B]$.

It is well-known (see Fishburn, 1970; Krantz et al., 1971) that there is a real-valued function $f$ on $A$ such that, for all $a, b \in A$,

$$
a T b \Leftrightarrow f(a) \geq f(b),
$$

if and only if $T$ is a weak order and there is a finite or countably infinite set $B \subseteq A$ that is dense in $A$ for $T$.

Let $T$ and $T^{\prime}$ be two weak orders on $A$. We say that $T^{\prime}$ refines $T$ if, for all $a, b \in A, a T^{\prime} b \Rightarrow a T b$. If $T^{\prime}$ refines $T$ and there is a set $B$ that is dense in $A$ for $T^{\prime}, B$ is also dense in $A$ for $T$. 


\section{$2.2 \quad$ The setting}

Let $n \geq 2$ be an integer and $X=X_{1} \times X_{2} \times \cdots \times X_{n}$ be a set of objects. Elements $x, y, z, \ldots$ of $X$ will be interpreted as alternatives evaluated on a set $N=\{1,2, \ldots, n\}$ of attributes. For any nonempty subset $J$ of the set of attributes $N$, we denote by $X_{J}\left(\operatorname{resp} . X_{-J}\right)$ the set $\prod_{i \in J} X_{i}\left(\operatorname{resp} . \prod_{i \notin J} X_{i}\right)$. With customary abuse of notation, $\left(x_{J}, y_{-J}\right)$ will denote the element $w \in X$ such that $w_{i}=x_{i}$ if $i \in J$ and $w_{i}=y_{i}$ otherwise. When $J=\{i\}$ we will simply write $X_{-i}$ and $\left(x_{i}, y_{-i}\right)$.

\subsection{Primitives}

Let $r \geq 2$ be an integer; we define $R=\{1,2, \ldots, r\}$ and $R^{+}=\{2,3, \ldots, r\}$. Our primitives consist in an $r$-fold partition $\left\langle C^{1}, C^{2}, \ldots, C^{r}\right\rangle$ of the set $X$ (the sets $C^{k}$ are therefore nonempty and pairwise disjoint; their union is the entire set $X)$. We often abbreviate $\left\langle C^{1}, C^{2}, \ldots, C^{r}\right\rangle$ as $\left\langle C^{k}\right\rangle_{k \in R}$. Note that throughout the paper superscripts are used to distinguish between categories and not, unless otherwise specified, to denote exponentiation.

We interpret the partition $\left\langle C^{k}\right\rangle_{k \in R}$ as the result of a sorting model between ordered categories applied to the alternatives in $X$. We suppose that the ordering of these categories is known beforehand and that they have been labelled in such a way that the desirability of a category increases with its label: the worst category is $C^{1}$ and the best one is $C^{r}$. Our central aim is to study various models allowing to represent the information contained in $\left\langle C^{k}\right\rangle_{k \in R}$.

\section{Remark 1}

The fact that we suppose the ordering of categories is known beforehand is in line with the type of data that is likely to be collected in order to test the conditions that will be introduced below. Furthermore, this does not involve any serious loss of generality.

Indeed, suppose that the ordering of categories is unknown and, consequently, that the categories have been labelled arbitrarily. In such a case, it will be extremely unlikely that the conditions introduced below are satisfied since they implicitly assume that categories have been labelled according to their desirability. In this case, we should reformulate our conditions saying that it is possible to relabel the categories in such a way that these conditions hold. This would clearly imply a much more cumbersome notation and formulation of the conditions with almost no additional insight. Hence, we stick to the framework in which the ordering of the categories is known and categories are labelled accordingly. 
For all $k \in R^{+}$, we define $C^{\geq k}=\bigcup_{j=k}^{r} C^{j}$ and $C^{<k}=\bigcup_{j=1}^{k-1} C^{j}$. The set $C^{\geq k}$ (resp. $C^{<k}$ ) is therefore a category grouping all categories that are at least as good as (resp. worse than) than $C^{k}$. The $r-1$ twofold partitions $\left\langle C^{\geq k}, C^{<k}\right\rangle$ for $k \in R^{+}$will play a central role in what follows.

We say that an attribute $i \in N$ is influent for $\left\langle C^{k}\right\rangle_{k \in R}$ if there are $x_{i}, y_{i} \in$ $X_{i}$ and $a_{-i} \in X_{-i}$ such that $\left(x_{i}, a_{-i}\right)$ and $\left(y_{i}, a_{-i}\right)$ do not belong to the same category. We say that an attribute is degenerate if it is not influent. Clearly, a degenerate attribute has no influence whatsoever on the sorting of the alternatives and may be suppressed from $N$. Henceforth, we suppose that all attributes are influent for $\left\langle C^{k}\right\rangle_{k \in R}$. It is important to notice that an attribute being influent for $\left\langle C^{k}\right\rangle_{k \in R}$ may be degenerate for some, but not all, of the twofold partitions $\left\langle C^{\geq k}, C^{<k}\right\rangle$.

\section{Remark 2}

The fact that not all attributes are influent for all twofold partitions $\left\langle C^{\geq k}, C^{<k}\right\rangle$ will complicate the analysis. The reader willing to have a feeling of the results without entering into details is invited to skip the parts linked with the treatment of degenerate attributes. He/she is also invited to devise the much simpler proofs that are available if it is supposed that all attributes are influent for all twofold partitions induced by $\left\langle C^{k}\right\rangle_{k \in R}$. This is a strong hypothesis however.

\section{A general measurement framework}

\subsection{The model}

In the context of categorization tasks in Psychology, Goldstein (1991) suggested the use of conjoint measurement techniques for the analysis of twofold and threefold partitions of a set of multi-attributed alternatives through what he called "decomposable threshold models". This analysis was independently rediscovered and generalized in Greco et al. (2001b) and Słowiński et al. (2002), in the context of MCDM, for the study of $r$-fold partitions. Let us notice that the analysis in these two papers should be viewed in the wider context of an original approach to MCDM based on an extension of rough set theory using dominance instead of indiscernibility relations (on this approach, see Greco et al., 1999a,b, 2001c, 2002b, 2005)

For the convenience of the reader and because our proofs are somewhat simpler than the one proposed in the above-mentioned papers, we briefly recall here some points of this analysis. The various alternative interpretations of this measurement model will be analyzed in the Section 3.2 
Consider a measurement model in which, for all $x \in X$ and all $k \in R$,

$$
x \in C^{k} \Leftrightarrow \sigma_{k}<F\left(u_{1}\left(x_{1}\right), u_{2}\left(x_{2}\right), \ldots, u_{n}\left(x_{n}\right)\right)<\sigma_{k+1},
$$

where $\sigma_{1}, \sigma_{2}, \ldots, \sigma_{r+1}$ are real numbers such that $\sigma_{1}<\sigma_{2}<\ldots<\sigma_{r+1}, u_{i}$ is a real-valued function on $X_{i}$ and $F$ is a real-valued function on $\prod_{i=1}^{n} u_{i}\left(X_{i}\right)$ that is increasing in all its arguments. The weakening of model $(D 1)$ in which $F$ is only supposed to be nondeacreasing in all its arguments will be called model (D2).

Define on each $X_{i}$ the binary relation $\succsim_{i}^{R}$ letting, for all $x_{i}, y_{i} \in X_{i}$,

$x_{i} \succsim_{i}^{R} y_{i} \Leftrightarrow$ [for all $a_{-i} \in X_{-i}$ and all $k \in R^{+},\left(y_{i}, a_{-i}\right) \in C^{k} \Rightarrow\left(x_{i}, a_{-i}\right) \in C^{\geq k}$ ],

the rationale for the superscript $R$ being that this relation depends on the entire partition $\left\langle C^{k}\right\rangle_{k \in R}$. We use $\succ_{i}^{R}$ and $\sim_{i}^{R}$ as is usual. By construction, $\succsim_{i}^{R}$ is reflexive and transitive. When $x_{i} \succsim_{i}^{R} y_{i}$, an alternative $\left(x_{i}, a_{-i}\right)$ must belong to a category that is at least as good as the category containing the alternative $\left(y_{i}, a_{-i}\right)$. Hence, $\succsim_{i}^{R}$ may be interpreted as an "at least as good as" relation induced on $X_{i}$ by the partition $\left\langle C^{k}\right\rangle_{k \in R}$. We have:

\section{Lemma 3}

For all $k \in R^{+}$and all $x, y \in X$,

1. $\left[y \in C^{k}\right.$ and $\left.x_{i} \succsim_{i}^{R} y_{i}\right] \Rightarrow\left(x_{i}, y_{-i}\right) \in C^{\geq k}$,

2. $\left[x_{i} \sim_{i}^{R} y_{i}\right.$, for all $\left.i \in N\right] \Rightarrow\left[x \in C^{k} \Leftrightarrow y \in C^{k}\right]$.

ProOF

Part 1 is clear from the definition of $\succsim_{i}^{R}$. Part 2 follows.

We say that the partition $\left\langle C^{k}\right\rangle_{k \in R}$ is $R$-linear on attribute $i \in N$ (condition linear $\left._{i}^{R}\right)$ if, for all $x_{i}, y_{i} \in X_{i}$, all $k, \ell \in R^{+}$and all $a_{-i}, b_{-i} \in X_{-i}$,

$$
\left.\begin{array}{l}
\left(x_{i}, a_{-i}\right) \in C^{k} \\
\text { and } \\
\left(y_{i}, b_{-i}\right) \in C^{\ell}
\end{array}\right\} \Rightarrow\left\{\begin{array}{c}
\left(y_{i}, a_{-i}\right) \in C^{\geq k} \\
\text { or } \\
\left(x_{i}, b_{-i}\right) \in C^{\geq \ell}
\end{array} \quad\left(\operatorname{linear}_{i}^{R}\right)\right.
$$

We say that $\left\langle C^{k}\right\rangle_{k \in R}$ is $R$-linear if it is $R$-linear on all attributes $i \in N$. $R$-linearity was considered by Goldstein (1991) for the case of twofold and threefold partitions. It was independently rediscovered and generalized in Greco et al. (2001b) for the analysis of $r$-fold partitions. The adaptation of this condition to the study of binary relations, first suggested by Goldstein (1991), is central in the analysis of the "nontransitive decomposable models" analyzed in Bouyssou and Pirlot (1999, 2002b, 2004a). 


\section{Remark 4}

Observe that, in the expression of linear ${ }_{i}^{R}$, it is possible to replace the premises $\left(x_{i}, a_{-i}\right) \in C^{k}$ and $\left(y_{i}, b_{-i}\right) \in C^{\ell}$ by $\left(x_{i}, a_{-i}\right) \in C^{\geq k}$ and $\left(y_{i}, b_{-i}\right) \in$ $C^{\geq \ell}$. Indeed, suppose that $\left(x_{i}, a_{-i}\right) \in C^{\geq k}$ and $\left(y_{i}, b_{-i}\right) \in C^{\geq \ell}$ while $\left(y_{i}, a_{-i}\right) \in$ $C^{<k}$ and $\left(x_{i}, b_{-i}\right) \in C^{<\ell}$. Since $\left(x_{i}, a_{-i}\right) \in C^{\geq k}$, we have $\left(x_{i}, a_{-i}\right) \in C^{\alpha}$, for some $\alpha \geq k$. Similarly, we have $\left(y_{i}, b_{-i}\right) \in C^{\beta}$, for some $\beta \geq \ell$. Applying linear $_{i}^{R}$ to $\left(x_{i}, a_{-i}\right) \in C^{\alpha}$ and $\left(y_{i}, b_{-i}\right) \in C^{\beta}$ leads to either $\left(y_{i}, a_{-i}\right) \in C^{\geq \alpha}$ or $\left(x_{i}, b_{-i}\right) \in C^{\geq \beta}$. Because $\alpha \geq k$ and $\beta \geq \ell$, we know this implies either $\left(y_{i}, a_{-i}\right) \in C^{\geq k}$ or $\left(x_{i}, b_{-i}\right) \in C^{\geq \ell}$, a contradiction.

Greco et al. (2001b) and Słowiński et al. (2002) give several other reformulations of this condition. Since they are not important for our purposes, we refer the interested reader to these two papers.

The consequences of $R$-linearity on attribute $i \in N$ are noted below.

\section{Lemma 5}

A partition $\left\langle C^{k}\right\rangle_{k \in R}$ satisfies linear ${ }_{i}^{R}$ iff $\succsim_{i}^{R}$ is complete.

Proof

The partition $\left\langle C^{k}\right\rangle_{k \in R}$ violates linear ${ }_{i}^{R}$ on $i \in N$ if and only if, for some $x_{i}, y_{i} \in X_{i}$, some $k, \ell \in R^{+}$, and some $a_{-i}, b_{-i} \in X_{-i},\left(x_{i}, a_{-i}\right) \in C^{k}$, $\left(y_{i}, b_{-i}\right) \in C^{\ell},\left(y_{i}, a_{-i}\right) \notin C^{\geq k}$ and $\left(x_{i}, b_{-i}\right) \notin C^{\geq \ell}$. This is equivalent to saying that $\succsim_{i}^{R}$ is not complete.

The following lemma shows that $R$-linear is a necessary condition for model (D2) and connects the functions $u_{i}$ in this model with the relations $\succsim_{i}^{R}$.

\section{Lemma 6}

1. If $\left\langle C^{k}\right\rangle_{k \in R}$ has a representation in model (D2) then it is $R$-linear.

2. If $\langle\mathscr{A}, \mathscr{U}\rangle$ has a representation in model (D2) then,

$$
x_{i} \succ_{i}^{R} y_{i} \Rightarrow u_{i}\left(x_{i}\right)>u_{i}\left(y_{i}\right) .
$$

Proof

Part 1. Suppose that $\left(x_{i}, a_{-i}\right) \in C^{k}$ and $\left(y_{i}, b_{-i}\right) \in C^{\ell}$, so that

$$
\begin{aligned}
& \sigma_{k}<F\left(u_{1}\left(a_{1}\right), \ldots, u_{i-1}\left(a_{i-1}\right), u_{i}\left(x_{i}\right), u_{i+1}\left(a_{i+1}\right), \ldots, u_{n}\left(a_{n}\right)\right)<\sigma_{k+1} \text { and } \\
& \sigma_{\ell}<F\left(u_{1}\left(b_{1}\right), \ldots, u_{i-1}\left(b_{i-1}\right), u_{i}\left(y_{i}\right), u_{i+1}\left(b_{i+1}\right), \ldots, u_{n}\left(b_{n}\right)\right)<\sigma_{\ell+1} .
\end{aligned}
$$

We have either $u_{i}\left(y_{i}\right) \geq u_{i}\left(x_{i}\right)$ or $u_{i}\left(x_{i}\right) \geq u_{i}\left(y_{i}\right)$. Using the nondecreasingness of $F$, this implies either

$$
\begin{aligned}
& \sigma_{k}<F\left(u_{1}\left(a_{1}\right), \ldots, u_{i-1}\left(a_{i-1}\right), u_{i}\left(y_{i}\right), u_{i+1}\left(a_{i+1}\right), \ldots, u_{n}\left(a_{n}\right)\right) \text { or } \\
& \sigma_{\ell}<F\left(u_{1}\left(b_{1}\right), \ldots, u_{i-1}\left(b_{i-1}\right), u_{i}\left(x_{i}\right), u_{i+1}\left(b_{i+1}\right), \ldots, u_{n}\left(b_{n}\right)\right) .
\end{aligned}
$$


Hence, model $(D 2)$ implies that we have either $\left(y_{i}, a_{-i}\right) \in C^{\geq k}$ or $\left(x_{i}, b_{-i}\right) \in$ $C^{\geq \ell}$, as required by linear $_{i}^{R}$.

Part 2. Suppose that $u_{i}\left(x_{i}\right) \leq u_{i}\left(y_{i}\right)$. Using the nondecreasingness of $F$, $\sigma_{k}<F\left(u_{1}\left(a_{1}\right), \ldots, u_{i-1}\left(a_{i-1}\right), u_{i}\left(x_{i}\right), u_{i+1}\left(a_{i+1}\right), \ldots, u_{n}\left(a_{n}\right)\right)<\sigma_{k+1}$ implies $\sigma_{k}<F\left(u_{1}\left(a_{1}\right), \ldots, u_{i-1}\left(a_{i-1}\right), u_{i}\left(y_{i}\right), u_{i+1}\left(a_{i+1}\right), \ldots, u_{n}\left(a_{n}\right)\right)$.

This shows that, for all $a_{-i} \in X_{-i},\left(x_{i}, a_{-i}\right) \in C^{k}$ implies $\left(y_{i}, a_{-i}\right) \in C^{\geq k}$, so that $y_{i} \succsim_{i}^{R} x_{i}$.

Omitting the cumbersome formulation of the order denseness condition in terms of the partition $\left\langle C^{k}\right\rangle_{k \in R}$, this leads the following result:

\section{Proposition 7}

A partition $\left\langle C^{k}\right\rangle_{k \in R}$ has a representation in model (D1) iff it is $R$-linear and, for all $i \in N$, there is a finite or countably infinite set $X_{i}^{\prime} \subseteq X_{i}$ that is dense in $X_{i}$ for $\succsim_{i}^{R}$. Furthermore:

- if $\left\langle C^{k}\right\rangle_{k \in R}$ has a representation in model $(D 1)$, it has a representation in which, for all $i \in N, u_{i}$ is a numerical representation of $\succsim_{i}^{R}$,

- models (D1) and (D2) are equivalent.

\section{Proof}

The necessity of $R$-linearity for model $(D 2)$ results from Part 1 of Lemma 6 . Part 2 of Lemma 6 shows that, in model (D2), the weak order induced on $X_{i}$ by $u_{i}$ always refines $\succsim_{i}^{R}$. Hence, there is a finite or countably infinite set $X_{i}^{\prime} \subseteq X_{i}$ that is dense in $X_{i}$ for $\succsim_{i}^{R}$.

Let us show that the conditions imply model $(D 1)$, which will also shows that models $(D 1)$ and $(D 2)$ are equivalent. Using Lemma 5, we know that $\succsim_{i}^{R}$ is a weak order. Since there is a finite or countably infinite set $X_{i}^{\prime} \subseteq X_{i}$ that is dense in $X_{i}$ for $\succsim_{i}^{R}$, there is a real-valued function $u_{i}$ on $X_{i}$ such that, for all $x_{i}, y_{i} \in X_{i}$,

$$
x_{i} \succsim_{i}^{R} y_{i} \Leftrightarrow u_{i}\left(x_{i}\right) \geq u_{i}\left(y_{i}\right)
$$

Consider, on each $i \in N$ any function $u_{i}$ satisfying (2) and take any $\sigma_{1}, \sigma_{2}$, $\ldots, \sigma_{r+1} \in \mathbb{R}$ such that $\sigma_{1}<\sigma_{2}<\cdots<\sigma_{r+1}$. For all $k \in R$, consider any increasing function $\phi_{k}$ mapping $\mathbb{R}$ into $\left(\sigma_{k}, \sigma_{k+1}\right)$. Define $F$ on $\prod_{i=1}^{n} u_{i}\left(X_{i}\right)$ letting, for all $x \in X$ and all $k \in R$,

$$
F\left(u_{1}\left(x_{1}\right), u_{2}\left(x_{2}\right), \ldots, u_{n}\left(x_{n}\right)\right)=\phi_{k}\left(\sum_{i=1}^{n} u_{i}\left(x_{i}\right)\right) \text { if } x \in C^{k} .
$$

The well-definedness of $F$ follows from Part 2 of Lemma 3. Its increasingness is easily shown using the definition of $u_{i}$ and Part 1 of Lemma 3. 
A version of this result for the case of two or three category appears in Goldstein (1991). Greco et al. (2001b) and Słowiński et al. (2002) state a version of this result when $X$ is finite or countably infinite.

The uniqueness of the representation of $\left\langle C^{k}\right\rangle_{k \in R}$ in model $(D 1)$ is quite weak. It can easily be analyzed along the lines sketched in Bouyssou and Marchant (2005).

Model $(D 1)$ contains as particular cases many sorting models that have been proposed in the literature. Notice, in particular, that when $F$ is taken to be a sum, model $(D 1)$ is nothing but the additive sorting model used in the UTADIS technique (Jacquet-Lagrèze, 1995; Zopounidis and Doumpos, 2000b). As shown below, it also contains the pessimistic version of ELECTRE TRI as a particular case. We analyse in the next section several alternative equivalent interpretations of this model proposed in Greco et al. (2001b) and Słowiński et al. (2002).

\section{Remark 8}

The above proof shows that model $(D 1)$ may equivalently be written replacing one of the two strict inequalities by a non strict one, e.g., letting, for all $x \in X$,

$$
x \in C^{k} \Leftrightarrow \sigma_{k} \leq F\left(u_{1}\left(x_{1}\right), u_{2}\left(x_{2}\right), \ldots, u_{n}\left(x_{n}\right)\right)<\sigma_{k+1} .
$$

In model $(D 2)$, it is always possible to take $F$ to be a constant on each of the categories $C^{k}$, using functions $\phi_{k}$ mapping $\mathbb{R}$ to $\rho_{k}$ with $\sigma_{k}<\rho_{k}<\sigma_{k+1}$. With such a representation, $F$ is also a numerical representation of the weak order that is naturally induced on $X$ by $\left\langle C^{k}\right\rangle_{k \in R}$.

\subsection{Interpretations of model $(D 1)$}

The framework offered by model $(D 1)$ is quite flexible. Greco et al. (2001b, Theorem 2.1, parts 3 and 4$)^{1}$ have proposed two equivalent reformulations of model $(D 1)$.

The first model suggested by Greco et al. (2001b, Theorem 2.1, Part 4) uses "at least" decision rules ${ }^{2}$. In this model, a complete and transitive relation $\mathcal{S}_{i}$ is supposed to be defined on each $X_{i}$. A decision rule $d$ consists in a subset $N^{d} \subseteq N$ of attributes and, for each $i \in N^{d}$, an element $\delta_{i}^{d} \in X_{i}$. The syntax of the "at least" decision rule $d$ is the following:

$$
\left[x_{i} \mathcal{S}_{i} \delta_{i}^{d}, \forall i \in N^{d}\right] \Rightarrow x \in C^{\geq k} .
$$

\footnotetext{
${ }^{1}$ Closely related results appear, without proof, in Słowiński et al. (2002, Theorem 2.1).

${ }^{2}$ It is also be possible to use, equivalently, what Greco et al. (2001b) call "at most" decision rules.
} 
A set of decision rules $\mathcal{D}$ is said to represent $\left\langle C^{k}\right\rangle_{k \in R}$ if,

- for each $x \in C^{k}$ with $k \in R^{+}$

- there is at least one decision rule in $d \in \mathcal{D}$ that matches $x$ (i.e., such that $x$ satisfies the premises of $\left.d:\left[x_{i} \mathcal{S}_{i} \delta_{i}^{d}, \forall i \in N^{d}\right]\right)$ and assigns $x$ to $C^{\geq k}$,

- there is no rule in $\mathcal{D}$ that matches $x$ and assigns $x$ to $C^{\geq \ell}$ with $\ell>k$,

- $x \in C^{1}$ if there is no decision rule in $\mathcal{D}$ that matches $x$.

Greco et al. (2001b) have argued that a model based on decision rules may be preferable to a model based on a functional representation, in terms of simplicity and transparency (this fact is at the heart of the "rough set approach" to MCDM problems as presented in Greco et al. 1999b, 2002b, 2005). Greco et al. (2001b, Theorem 2.1, Part 4) show that the decision rule model holds iff $\left\langle C^{k}\right\rangle_{k \in R}$ is $R$-linear.

\section{Remark 9}

Because the proof of the above fact is simple and may not be easily accessible, we recall its main steps below.

It is clear that the "at least" decision rule model implies $R$-linearity. Indeed suppose that $\left(x_{i}, a_{-i}\right) \in C^{k}$ and $\left(y_{i}, b_{-i}\right) \in C^{\ell}$. If $k=1$ or $\ell=1$, there is nothing to prove. Suppose henceforth that $k>1$ and $\ell>1$. Therefore, $\left(x_{i}, a_{-i}\right)$ is matched by rule $d^{1} \in \mathcal{D}$ that assigns it to $C^{\geq k}$ and there is no rule matching $x$ assigning it to a higher category. Similarly, $\left(y_{i}, b_{-i}\right)$ is matched by rule $d^{2} \in \mathcal{D}$ that assigns it to $C^{\geq \ell}$ and there is no rule matching $y$ assigning it to a higher category.

If $i \notin N^{d^{1}}$ or if $i \notin N^{d^{2}}$, it is clear that $R$-linearity cannot be violated. Suppose therefore that $i \in N^{d^{1}}$ and $i \in N^{d^{2}}$. Since the relations $\mathcal{S}_{i}$ are complete, we have either $x_{i} \mathcal{S}_{i} y_{i}$ or $y_{i} \mathcal{S}_{i} x_{i}$. Because $\left(x_{i}, a_{-i}\right)$ is matched by rule $d^{1} \in \mathcal{D}$ and $\left(y_{i}, b_{-i}\right)$ is matched by rule $d^{2} \in \mathcal{D}$, we know that $x_{i} \mathcal{S}_{i} \delta_{i}^{d^{1}}$ and $y_{i} \mathcal{S}_{i} \delta_{i}^{d^{2}}$. Because $\mathcal{S}_{i}$ are transitive, we have either that $y_{i} \mathcal{S}_{i} \delta_{i}^{d^{1}}$ or $x_{i} \mathcal{S}_{i} \delta_{i}^{d^{2}}$. Hence, either $\left(y_{i}, a_{-i}\right)$ is matched by rule $d^{1} \in \mathcal{D}$ or $\left(x_{i}, b_{-i}\right)$ is matched by rule $d^{2} \in \mathcal{D}$. This implies either $\left(y_{i}, a_{-i}\right) \in C^{\geq k}$ or $\left(x_{i}, b_{-i}\right) \in C^{\geq \ell}$, and linear ${ }_{i}^{R}$ holds.

Conversely, suppose that $\left\langle C^{k}\right\rangle_{k \in R}$ is $R$-linear. Using Lemma 5, we know that $\succsim_{i}^{R}$ is complete and we take, for all $i \in N, \mathcal{S}_{i}=\succsim_{i}^{R}$. For each $x \in C^{k}$ define an "at least" decision $d^{x}$ saying that

$$
\left[y_{i} \mathcal{S}_{i} x_{i}, \forall i \in N\right] \Rightarrow y \in C^{\geq k} .
$$


It is clear that each $x \in C^{k}$ is matched by rule $d^{x}$ assigning it to $C^{\geq k}$. Suppose that there is a rule $d \in \mathcal{D}$ that matches $x$ and that assigns it to $C^{\geq \ell}$ with $\ell>k$. This would imply that there is a $y \in C^{\ell}$ such that $x_{i} \succsim_{i}^{R} y_{i}$, for all $i \in N$. Using Lemma 3, this implies $x \in C^{\geq \ell}$. This is contradictory since $x \in C^{k}$ and $\ell>k$.

We refer the reader to Greco et al. (2001b) and to Słowiński et al. (2002) for an in depth study of the decision rule model for sorting and several of its extensions.

The second model (henceforth the "relational model") is based on binary relations. In this model, a complete and transitive relation $\mathcal{S}_{i}$ is supposed to be defined on each $X_{i}$. A reflexive binary relation $\mathcal{S}$ is defined on $X$ in such a way that it is compatible with the relations $\mathcal{S}_{i}$, i.e., such that, for all $x, y \in X$, all $i, j \in N$, all $z_{i} \in X_{i}$ and all $w_{j} \in X_{j}$,

$$
\left[x \mathcal{S} y, z_{i} \mathcal{S}_{i} x_{i}, y_{j} \mathcal{S}_{j} w_{j}\right] \Rightarrow\left(z_{i}, x_{-i}\right) \mathcal{S}\left(w_{j}, y_{-j}\right) .
$$

This expresses the fact that $\mathcal{S}$ is compatible with the dominance relation derived from the relations $\mathcal{S}_{i}$ (for a general study of such relations, see Bouyssou and Pirlot, 2004b). The relation $\mathcal{S}$ on $X$ is used to assign alternative to categories through their comparison with particular elements of $X$, interpreted as the lower limiting profiles of the categories. More precisely, the relational model is such that:

$$
x \in C^{\geq k} \Leftrightarrow x \mathcal{S} \pi^{k}
$$

where $\pi^{k} \in X$ is interpreted as the lower limiting profile of category $C^{k}$.

Greco et al. (2001b, Theorem 2.1, Part 3) have shown that the relational model holds iff $\left\langle C^{k}\right\rangle_{k \in R}$ is $R$-linear.

\section{Remark 10}

We present below a simple proof of the above fact.

It is easy to show that the relational model implies $R$-linearity. Indeed suppose that $\left(x_{i}, a_{-i}\right) \in C^{k}$ and $\left(y_{i}, b_{-i}\right) \in C^{\ell}$, so that $\left(x_{i}, a_{-i}\right) \mathcal{S} \pi^{k}$ and $\left(y_{i}, b_{-i}\right) \mathcal{S} \pi^{\ell}$. Since the relations $\mathcal{S}_{i}$ are complete, we have either $x_{i} \mathcal{S}_{i} y_{i}$ or $y_{i} \mathcal{S}_{i} x_{i}$. Using (3), this implies that either $\left(y_{i}, a_{-i}\right) \mathcal{S} \pi^{k}$ or $\left(x_{i}, b_{-i}\right) \mathcal{S} \pi^{\ell}$. Hence, we have either $\left(y_{i}, a_{-i}\right) \in C^{\geq k}$ or $\left(x_{i}, b_{-i}\right) \in C^{\geq \ell}$, so that linear ${ }_{i}^{R}$ holds.

Conversely ${ }^{3}$, suppose that $\left\langle C^{k}\right\rangle_{k \in R}$ is $R$-linear. We know that the relations $\succsim_{i}^{R}$ are weak orders and we take, for all $i \in N, \mathcal{S}_{i}=\succsim_{i}^{R}$.

Define a binary relation $R$ on $X$ letting, for all $x, y \in X, x R y$ iff $\left[x \in C^{k}\right.$ and $x \in C^{\ell}$ with $\left.k \geq \ell\right]$. It is easy to see that $R$ is a weak order on

\footnotetext{
${ }^{3}$ Our proof differs from the one proposed in Greco et al. (2001b). When $X$ is not finite, the proof proposed by Greco et al. (2001b) would need to be adapted.
} 
$X$ having $r$ equivalence classes. Take $\mathcal{S}=R$. Define $\pi^{k} \in X$ to be any element of $C^{k}$. We clearly have $x \in C^{\geq k} \Leftrightarrow x \mathcal{S} \pi^{k}$. It is easy to see that, with such definitions, (3) holds.

\section{Remark 11}

In Greco et al. (2001b) and Słowiński et al. (2002), it is asserted, but not proved, that the profiles $\pi^{k}$ in the relational model may always be chosen in such a way that, for all $k \in R^{+}$and all $i \in N$, we have:

$$
\pi_{i}^{k+1} \mathcal{S}_{i} \pi_{i}^{k}
$$

(profiles satisfying (5) will be called "regular profiles"). This claim is not correct.

Consider any partition $\left\langle C^{k}\right\rangle_{k \in R}$ that is $R$-linear. Observe that the complete and transitive relations $\mathcal{S}_{i}$ in the relational model must always be such that $\succsim_{i}^{R} \subseteq \mathcal{S}_{i}$. Suppose indeed that $x_{i} \mathcal{S}_{i} y_{i}$ and that $\operatorname{Not}\left[x_{i} \succsim_{i}^{R} y_{i}\right]$. This last relation implies that, for some $k \in R^{+}$and some $a_{-i} \in X_{-i},\left(y_{i}, a_{-i}\right) \in C^{k}$ and $\left(x_{i}, a_{-i}\right) \in C^{<k}$. Using the relational model, we obtain $\left(y_{i}, a_{-i}\right) \mathcal{S} \pi^{k}$. Using (3), this would imply $\left(x_{i}, a_{-i}\right) \mathcal{S} \pi^{k}$, so that $\left(x_{i}, a_{-i}\right) \in C^{\geq k}$, a contradiction. Hence, when $\succsim_{i}^{R}$ is a total order, we must have that $\succsim_{i}^{R}=\mathcal{S}_{i}$.

Observe that in the relational model we must have that $\pi^{k} \in C^{\geq k}$, since $\mathcal{S}$ is reflexive. Furthermore, it is impossible that $\pi^{k}=\pi^{\ell}$ with $k>\ell$. Indeed, taking any $x \in C^{\ell}$ we would have $x \mathcal{S} \pi^{\ell}$, so that $x \mathcal{S} \pi^{k}$, which would imply $x \in C^{\geq k}$. Consider now the following example.

\section{Example 12}

Suppose that $n=3, X_{1}=\left\{x_{1}, y_{1}\right\}, X_{2}=\left\{x_{2}, y_{2}\right\}$ and $X_{3}=\left\{x_{3}, y_{3}\right\}$. We consider a 4-fold partition $\left\langle C^{1}, C^{2}, C^{3}, C^{4}\right\rangle$ such that $C^{4}=\left\{\left(x_{1}, x_{2}, x_{3}\right),\left(y_{1}, x_{2}\right.\right.$, $\left.\left.x_{3}\right)\right\}, C^{3}=\left\{\left(x_{1}, y_{2}, x_{3}\right)\right\}, C^{2}=\left\{\left(x_{1}, x_{2}, y_{3}\right)\right\}$ and $C^{1}$ containing all remaining alternatives. It is easy to see that we have $x_{i} \succ_{i}^{R} y_{i}$, for all $i \in N$. This shows that the partition $\left\langle C^{1}, C^{2}, C^{3}, C^{4}\right\rangle$ is $R$-linear.

Suppose now that the above partition has a representation in the relational model. We must take $\succsim_{i}^{R}=\mathcal{S}_{i}$, for all $i \in N$. Because $\pi^{k} \in C^{\geq k}$, we must take $\pi^{4}$ to be either $\left(x_{1}, x_{2}, x_{3}\right)$ or $\left(y_{1}, x_{2}, x_{3}\right)$.

Suppose that we take $\pi^{4}=\left(y_{1}, x_{2}, x_{3}\right)$. Since we must have $\pi^{3} \in C^{\geq 3}$ and $\pi^{3} \neq \pi^{4}$, we have either $\pi^{3}=\left(x_{1}, x_{2}, x_{3}\right)$ or $\pi^{3}=\left(x_{1}, y_{2}, x_{3}\right)$. In either case, (5) is violated.

Suppose now that we take $\pi^{4}=\left(x_{1}, x_{2}, x_{3}\right)$. We have either $\pi^{3}=$ $\left(y_{1}, x_{2}, x_{3}\right)$ or $\pi^{3}=\left(x_{1}, y_{2}, x_{3}\right)$. Suppose first that $\pi^{3}=\left(y_{1}, x_{2}, x_{3}\right)$. We must take either $\pi^{2}=\left(x_{1}, y_{2}, x_{3}\right)$ or $\pi^{2}=\left(x_{1}, x_{2}, y_{3}\right)$. In either case, (5) is violated. Suppose now that $\pi^{3}=\left(x_{1}, y_{2}, x_{3}\right)$. We must take either $\pi^{2}=\left(y_{1}, x_{2}, x_{3}\right)$ or $\pi^{2}=\left(x_{1}, x_{2}, y_{3}\right)$. In either case, (5) is violated. 
Therefore there is no representation of $\left\langle C^{1}, C^{2}, C^{3}, C^{4}\right\rangle$ in the relational model such that (5) holds.

The models that we will study in Sections 5 and 6 will not make use of profiles. Let us however show that how to modify the relational model in order to ensure that the profiles can always be defined so that (5) holds.

In, what we will call the "relational model with nested relations and regular profiles", a complete and transitive relation $\mathcal{S}_{i}$ is supposed to be defined on each $X_{i}$. For each $k \in R^{+}$, a reflexive binary relation $\mathcal{S}^{k}$ is defined on $X$. It is supposed that each relation $\mathcal{S}^{k}$ satisfies (3) and that the relations $\mathcal{S}^{k}$ are nested, i.e., such that:

$$
\mathcal{S}^{r} \subseteq \mathcal{S}^{r-1} \subseteq \cdots \subseteq \mathcal{S}^{2}
$$

For each $k \in R^{+}$, we define a profile $\pi^{k} \in X$ in such a way that (5) holds.

In the relational model with nested relations and regular profiles, we have:

$$
x \in C^{\geq k} \Leftrightarrow x \mathcal{S}^{k} \pi^{k}
$$

A particular case of the relational model with nested relations and regular profiles is obtained if we require all profiles $\pi^{2}, \pi^{3}, \ldots, \pi^{r}$ to be identical. We call this model the "relational model with nested relations and unique profile".

Compared to the relational model of Greco et al. (2001b), the relational model with nested relations and regular profiles proposed here uses several nested binary relations to assign alternative to categories, instead of just one in the relational model, but requires that the profiles dominate each other. We have:

\section{Proposition 13}

A partition $\left\langle C^{k}\right\rangle_{k \in R}$ has a representation in the relational model with nested relations and regular profiles iff it is $R$-linear. The relational model with nested relations and regular profiles is equivalent to the relational model with nested relations and unique profile.

PROOF

Necessity. Suppose that $\left(x_{i}, a_{-i}\right) \in C^{k}$ and $\left(y_{i}, b_{-i}\right) \in C^{\ell}$, so that $\left(x_{i}, a_{-i}\right) \mathcal{S}^{k}$ $\pi^{k}$ and $\left(y_{i}, b_{-i}\right) \mathcal{S}^{\ell} \pi^{\ell}$. Since the relations $\mathcal{S}_{i}$ are complete, we have either $x_{i} \mathcal{S}_{i} y_{i}$ or $y_{i} \mathcal{S}_{i} x_{i}$. Using (3), this implies that either $\left(y_{i}, a_{-i}\right) \mathcal{S}^{k} \pi^{k}$ or $\left(x_{i}, b_{-i}\right) \mathcal{S}^{\ell} \pi^{\ell}$. Hence, we have either $\left(y_{i}, a_{-i}\right) \in C^{\geq k}$ or $\left(x_{i}, b_{-i}\right) \in C^{\geq \ell}$, so that linear $_{i}^{R}$ holds.

Sufficiency. Suppose that $\left\langle C^{k}\right\rangle_{k \in R}$ is $R$-linear. We know that the relations $\succsim_{i}^{R}$ are weak orders and we take, for all $i \in N, \mathcal{S}_{i}=\succsim_{i}^{R}$. 
For all $k \in R^{+}$, define a relation $R^{k}$ on $X$ letting, for all $x, y \in X$, $x R^{k} y$ iff $\left[\left[x \in C^{\geq k}\right.\right.$ and $\left.y \in C^{\geq k}\right]$ or $\left[x \in C^{\ell}\right.$ and $y \in C^{\ell^{\prime}}$ with $\left.\left.k>\ell \geq \ell^{\prime}\right]\right]$. It is easy to see that $R^{k}$ is a weak order having $k$ equivalence classes. The first equivalence class of $R^{k}$ contains all alternatives in $C^{\geq k}$. The other equivalence classes consist of the categories lower than $k$ that are ordered in the natural way. We take, for all $k \in R^{+}, \mathcal{S}^{k}=R^{k}$, so that (6) clearly holds. Using the definition of $\succsim_{i}^{R}$, it is clear that (3) holds, for all $k \in R^{+}$.

We take $\pi^{r}=\pi^{r-1}=\cdots=\pi^{2}$ to be equal to an arbitrary element of $C^{r}$. With such a definition, (5) trivially holds. It is easy to see that we have $x \in C^{\geq k} \Leftrightarrow x \mathcal{S}^{k} \pi^{k}$.

Observe that we have built above a representation of $\left\langle C^{k}\right\rangle_{k \in R}$ in the relational model with nested relations and unique profile. This proves the last part of the proposition.

Because the relational model of Greco et al. (2001b) does not always lead to using regular profiles, we consider that the relational model with nested relations and regular profiles is an attractive alternative to it. With this model in mind, we will suggest below a slight variant of ELECTRE TRI. Nevertheless, it is clear that the relational model of Greco et al. (2001b), the relational model with nested relations and regular profiles and the relational model with nested relations and unique profile are all equivalent.

\section{ELECTRE TRI}

For the ease of future reference, we briefly recall here the main points of the ELECTRE TRI sorting technique. We suppose below that preference and indifference thresholds are equal and that discordance effects occur in an "all or nothing" way. This will allow to keep things simple while preserving what we believe to be the general spirit of the method. Furthermore, for reasons detailed in Bouyssou and Marchant (2005), we restrict our attention to the pessimistic version of the method. We refer the reader to Mousseau et al. (2000b), Roy and Bouyssou (1993, ch. 6) or Wei (1992) for more detailed presentations.

The aim of ELECTRE TRI is to sort alternatives evaluated on several attributes between $r$ ordered categories $C^{1}, C^{2}, \ldots, C^{r}$. This is done as follows. For all $k \in R^{+}$, there is a profile $p^{k}$ being the lower limit of category $C^{k}$ and the upper limit of $C^{k-1}$. Each of these profiles $p^{k}$ is defined by its evaluations $\left(p_{1}^{k}, p_{2}^{k}, \ldots, p_{n}^{k}\right)$ on the attributes in $N$. Define $\widehat{X}_{i}=X_{i} \cup\left\{p_{i}^{2}, p_{i}^{3}, \ldots, p_{i}^{r}\right\}$ and $\widehat{X}=\prod_{i=1}^{n} \widehat{X}_{i}$. 
A semiorder $S_{i}$ is supposed to be defined on $\widehat{X}_{i}$. We note $P_{i}$ the asymmetric part of $S_{i}$ and $T_{i}$ the weak order underlying $S_{i}$, i.e., we have $x_{i} T_{i} y_{i}$ iff $\left[\left[z_{i} S_{i} x_{i} \Rightarrow z_{i} S_{i} x_{i}\right]\right.$ and $\left[y_{i} S_{i} z_{i} \Rightarrow x_{i} S_{i} z_{i}\right]$, for all $\left.z_{i} \in \widehat{X}_{i}\right]$. The relation $S_{i}$ is interpreted as an "at least as good" relation on $\widehat{X}_{i}$. Because categories are supposed to be ordered, it seems obvious to require that the definition of the profiles $p^{k}$ is such that, for all $i \in N$,

$$
p_{i}^{r} T_{i} p_{i}^{r-1} T_{i} \ldots T_{i} p_{i}^{2} .
$$

A strict semiorder $V_{i}$ included in $P_{i}$ is supposed to be defined on $\widehat{X}_{i}$. It is interpreted as a "far better than" relation on $\widehat{X}_{i}$. We suppose ${ }^{4}$ that $x_{i} T_{i} y_{i}$ and $y_{i} V_{i} z_{i}$ imply $x_{i} V_{i} z_{i}$ and that $z_{i} T_{i} w_{i}$ and $y_{i} V_{i} z_{i}$ imply $y_{i} V_{i} w_{i}$, so that the weak order underlying the strict semiorder $V_{i}$ is compatible with $T_{i}$.

For all $k \in R^{+}$, let $\lambda$ be a real number between $1 / 2$ and 1 . A nonnegative weight $w_{i}$ is assigned to each attribute $i \in N$. It is supposed that weights are normalized so that $\sum_{i=1}^{n} w_{i}=1$.

In ELECTRE TRI, we build a binary relation $S$ on $\widehat{X}$ letting, for all $x, y \in \widehat{X}$, (notice that it would be enough to define $S$ as a relation between the sets $X$ and $\left.\left\{p^{r}, p^{r-1}, \ldots, p^{2}\right\}\right)$,

$$
x S y \Leftrightarrow\left[\sum_{i \in S(x, y)} w_{i} \geq \lambda \text { and }\left[N o t\left[y_{i} V_{i} x_{i}\right], \text { for all } i \in N\right]\right] \text {, }
$$

where $S(x, y)=\left\{i \in N: x_{i} S_{i} y_{i}\right\}$. Hence, we have $x S y$ when $x$ is judged "at least as good as" $y$ on a qualified weighted majority of attributes (concordance condition) and there is no attribute on which $y$ is judged "far better" than $x$ (non-discordance condition).

The sorting of an alternative $x \in X$ is based on the comparison of $x$ with the profiles $p^{k}$ using the relation $S$. In the pessimistic version of ELECTRE TRI, we have, for all $x \in X$ and all $k \in R^{+}$,

$$
x \in C^{\geq k} \Leftrightarrow x S p^{k} .
$$

\section{Remark 14}

In the original presentation of the method (see Roy and Bouyssou, 1993, p. 390), the assignment of an alternative to one of the categories is presented

\footnotetext{
${ }^{4}$ This requirement is obviously satisfied when both $S_{i}$ and $V_{i}$ are defined using a realvalued function $g_{i}$ on $\widehat{X}_{i}$ together with indifference and veto thresholds, as in the usual presentation.
} 
slightly differently: for $k=r, r-1, \ldots 2$, it is tested whether $x S p^{k}$ and $x$ is assigned to the highest category $C^{k}$ such that this test is positive or to $C^{1}$ if the test is never positive. Our presentation is clearly equivalent to the original one.

\section{Remark 15}

ELECTRE TRI uses profiles that dominate each other in terms of the relations $S_{i}$. Compared to the relational models suggested earlier, it adds the additional flexibility to choose the profiles outside the set $X$. We will nevertheless show later that, neglecting additivity issues, if a partition can be obtained with ELECTRE TRI, it is always possible to obtain it using ELECTRE TRI profiles that belong to $X$.

For the moment, let us observe that the partition analyzed in Example 12 cannot be obtained with ELECTRE TRI.

Indeed, because $\left(x_{1}, x_{2}, x_{3}\right) \in C^{\geq 4},\left(x_{1}, y_{2}, x_{3}\right) \notin C^{\geq 4}$ and $\left(x_{1}, x_{2}, y_{3}\right) \notin$ $C^{\geq 4}$, we know that being at least as good as a profile on attributes $\{1,3\}$ or on attributes $\{1,2\}$ is not sufficient to establish outranking. Similarly because $\left(x_{1}, y_{2}, x_{3}\right) \in C^{\geq 3}$ and $\left(x_{1}, y_{2}, y_{3}\right) \notin C^{\geq 3}$, we know that being at least as good as a profile on attributes $\{2,3\}$ is not sufficient to establish outranking. Hence, to establish outranking it is necessary to be at least as good as the profile on all attributes.

Hence, since $\left(y_{1}, x_{2}, x_{3}\right) \in C^{4}$, we must have $y_{1} S_{1} p_{1}^{4}$. Because $\left(x_{1}, x_{2}, y_{3}\right) \in$ $C^{\geq 2}$ and $\left(y_{1}, x_{2}, y_{3}\right) \notin C^{\geq 2}$ we must have $\operatorname{Not}\left[y_{1} S_{1} p_{1}^{2}\right]$. But $y_{1} S_{1} p_{1}^{4}$ and $p_{1}^{4} T_{1} p_{1}^{2}$ implies $y_{1} S_{1} p_{1}^{2}$, a contradiction.

A seemingly very minor modification of ELECTRE TRI would allow it to be able to represent the partition analyzed in Example 12. Instead of considering a single binary relation $S$ it suffices, as in the relational model with nested relations and regular profiles proposed above, to consider several nested binary relations. This can easily be done as follows.

For all $k \in R^{+}$, let $\lambda^{k}$ be a real number between $1 / 2$ and 1 such that:

$$
\lambda^{r} \geq \lambda^{r-1} \geq \cdots \geq \lambda^{2} .
$$

For all $k \in R^{+}$, build a binary relation $S^{k}$ on $\widehat{X}$ letting, for all $x, y \in \widehat{X}$,

$$
x S^{k} y \Leftrightarrow\left[\sum_{i \in S(x, y)} w_{i} \geq \lambda^{k} \text { and }\left[\operatorname{Not}\left[y_{i} V_{i} x_{i}\right], \text { for all } i \in N\right]\right] \text {. }
$$

Observe that, by construction, we have:

$$
S^{r} \subseteq S^{r-1} \subseteq \cdots \subseteq S^{2} .
$$


The sorting of an alternative $x \in X$ is based on the comparison of $x$ with the profiles $p^{k}$ using the relations $S^{k}$. In the pessimistic version of ELECTRE TRI, we have, for all $x \in X$ and all $k \in R^{+}$,

$$
x \in C^{\geq k} \Leftrightarrow x S^{k} p^{k} .
$$

It is clear that this variant remains close to the original spirit of the authors of ELECTRE TRI. We will call it ELECTRE TRI with nested relations. Besides remaining close to the original method, this variant has larger descriptive ability than the priginal method. Indeed, it can represent the partition analyzed in Example 12. A routine check shows that this example can be obtained using ELECTRE TRI with nested relations using the following parameters:

$$
\begin{gathered}
x_{1} P_{1} y_{1} \quad x_{2} P_{2} y_{2} \quad x_{3} P_{3} y_{3}, \\
w_{1}=0.25 \quad w_{2}=0.35 \quad w_{3}=0.4, \\
p^{4}=p^{3}=p^{2}=\left(x_{1}, x_{2}, x_{3}\right), \\
\lambda_{4}=0,7 \quad \lambda_{3}=0.6 \quad \lambda_{2}=0.55, \\
V_{1}=V_{2}=V_{3}=\varnothing .
\end{gathered}
$$

\section{The noncompensatory sorting model}

This section studies a particular case of the sorting model (D1) that will turn to have close links with (the pessimistic version of) ELECTRE TRI in the absence of veto. As pointed to us by Salvatore Greco, Benedetto Matarazzo and Roman Słowiński, this model has intimate connections with the model based on the Sugeno integral studied in Greco et al. (2001b) and Słowiński et al. (2002). They will be studied in Section 5.5.

\subsection{Definitions}

We say that $\left\langle C^{k}\right\rangle_{k \in R}$ has a representation in the noncompensatory sorting model if:

(i) for all $i \in N$ there are sets $\mathscr{A}_{i}^{r} \subseteq \mathscr{A}_{i}^{r-1} \subseteq \cdots \subseteq \mathscr{A}_{i}^{k} \subseteq \cdots \subseteq \mathscr{A}_{i}^{2} \subseteq X_{i}$,

(ii) there are subsets $\mathscr{F}^{r}, \mathscr{F}^{r-1}, \ldots, \mathscr{F}^{k}, \ldots, \mathscr{F}^{2}$ of $2^{N}$ that are such that, for all $k \in R^{+}$and all $I, J \in 2^{N}$,

$$
\left[I \in \mathscr{F}^{k} \text { and } I \subseteq J\right] \Rightarrow J \in \mathscr{F}^{k}
$$


and are nested, i.e., such that,

$$
\mathscr{F}^{r} \subseteq \mathscr{F}^{r-1} \subseteq \cdots \subseteq \mathscr{F}^{2}
$$

such that:

$$
x \in C^{\geq k} \Leftrightarrow\left\{i \in N: x_{i} \in \mathscr{A}_{i}^{k}\right\} \in \mathscr{F}^{k},
$$

for all $x \in X$ and all $k \in R^{+}$. In this case, we say that $\left\langle\mathscr{F}^{k},\left\langle\mathscr{A}_{i}^{k}\right\rangle_{i \in N}\right\rangle$ is a representation of $\left\langle C^{k}\right\rangle_{k \in R}$ in the noncompensatory sorting model. We note $A^{k}(x)$ instead of $\left\{i \in N: x_{i} \in \mathscr{A}_{i}^{k}\right\}$ when there is no ambiguity on the underlying sets $\mathscr{A}_{i}^{k}$. We define, in Section $5, \mathscr{U}_{i}^{k}=X_{i} \backslash \mathscr{A}_{i}^{k}$.

The interpretation of the noncompensatory sorting model is simple. For all $k \in R^{+}$, we isolate within the set $X_{i}$ a subset $\mathscr{A}_{i}^{k}$ that we interpret as containing the elements of $X_{i}$ that are judged "satisfactory at the level $k$ ". In order for an alternative $x \in X$ to belong at least to $C^{k}$, it is necessary that the evaluations of $x$ are judged satisfactory at the level $k$ on a subset of attributes that is "sufficiently important at the level $k$ ", as indicated by the set $\mathscr{F}^{k}$. The fact that $\mathscr{F}^{k}$ satisfies (10) implies that replacing an unsatisfactory evaluation at the level $k$ by a satisfactory one cannot turn an alternative in $C^{k}$ into an alternative in $C^{<k}$. Because the categories are ordered, the hypothesis that $\mathscr{A}_{i}^{k} \subseteq \mathscr{A}_{i}^{k-1}$ simply means that an evaluation that is satisfactory at the level $k$ must be judged satisfactory at any lower level. Similarly imposing that $\mathscr{F}^{k} \subseteq \mathscr{F}^{k-1}$ means that a subset of attributes that is judged "sufficiently important at level $k$ " must be so at any lower level. It is easy to check that the above definition contains as a particular case the definition introduced in Bouyssou and Marchant (2005) for the case of two categories.

When no discordance is involved, i.e., when $V_{i}=\varnothing$, for all $i \in N$, the pessimistic version of ELECTRE TRI with nested relations (and, hence, the pessimistic version of ELECTRE TRI) is a particular case of the noncompensatory sorting model. Indeed, remember from Section 4 that, under the above hypotheses, we have in ELECTRE TRI with nested relations, for all $x \in X$ and all $k \in R^{+}$,

$$
x \in C^{\geq k} \Leftrightarrow \sum_{i \in S\left(x, p^{k}\right)} w_{i} \geq \lambda^{k} .
$$

Define $\mathscr{A}_{i}^{k}=\left\{i \in N: x_{i} S_{i} p^{k}\right\}$ and let $I \in \mathscr{F}^{k}$ whenever $\sum_{i \in I} w_{i} \geq \lambda^{k}$. By construction of the profiles $p^{k}$ and of the relations $S_{i}$, we have $\mathscr{A}_{i}^{k} \subseteq$ $\mathscr{A}_{i}^{k-1}$. Because $\lambda^{k+1} \geq \lambda^{k}$, we have $\mathscr{F}^{k+1} \subseteq \mathscr{F}^{k}$. Hence $\left\langle\mathscr{F}^{k},\left\langle\mathscr{A}_{i}^{k}\right\rangle_{i \in N}\right\rangle$ is a representation of $\left\langle C^{k}\right\rangle_{k \in R}$ in the noncompensatory sorting model.

Our aim in this section is to characterize the partitions $\left\langle C^{k}\right\rangle_{k \in R}$ that can be represented in the noncompensatory sorting model. 


\subsection{Axioms}

Let us first observe that a partition $\left\langle C^{k}\right\rangle_{k \in R}$ having a representation in the noncompensatory sorting model must be $R$-linear.

\section{Lemma 16}

If a partition $\left\langle C^{k}\right\rangle_{k \in R}$ has a representation in the noncompensatory sorting model then it is $R$-linear.

ProOF

Suppose that linear $_{i}^{R}$ is violated so that, for some $k, \ell \in R^{+}$, some $x_{i}, y_{i} \in X_{i}$, and some $a_{-i}, b_{-i} \in X_{-i},\left(x_{i}, a_{-i}\right) \in C^{k},\left(y_{i}, b_{-i}\right) \in C^{\ell},\left(y_{i}, a_{-i}\right) \in C^{<k}$ and $\left(x_{i}, b_{-i}\right) \in C^{<\ell}$. This implies $x_{i} \in \mathscr{A}_{i}^{k}, y_{i} \notin \mathscr{A}_{i}^{k}, y_{i} \in \mathscr{A}_{i}^{\ell}$ and $x_{i} \notin \mathscr{A}_{i}^{\ell}$, violating the fact that we have either $\mathscr{A}_{i}^{k} \subseteq \mathscr{A}_{i}^{\ell}$ or $\mathscr{A}_{i}^{\ell} \subseteq \mathscr{A}_{i}^{k}$.

In the noncompensatory sorting model all elements in $\mathscr{A}_{i}^{k}$ are treated in a similar way. Therefore, if $\left\langle C^{k}\right\rangle_{k \in R}$ has a representation in the noncompensatory sorting model, then, for all $i \in N$, the relation $\succsim_{i}^{R}$ can have at most $r$ distinct equivalence classes. Hence, for all $i \in N$, the set $X_{i} / \sim_{i}^{R}$ is finite. In view of Proposition 7 this shows that the noncompensatory sorting model is a particular case of model $(D 1)$.

Using, e.g., an additive sorting model, it is easy to build partitions $\left\langle C^{k}\right\rangle_{k \in R}$ in which all relations $\succsim_{i}^{R}$ have at most $r$ equivalence classes that cannot be represented in the noncompensatory sorting model. In order to capture the specific features of the noncompensatory sorting model, consider, for all $k \in R^{+}$and all $i \in N$, the binary relation $\succsim_{i}^{k}$ on $X_{i}$ such that, for all $x_{i}, y_{i} \in X_{i}$,

$$
\left.x_{i} \succsim_{i}^{k} y_{i} \Leftrightarrow \text { for all } a_{-i} \in X_{-i},\left(y_{i}, a_{-i}\right) \in C^{k} \Rightarrow\left(x_{i}, a_{-i}\right) \in C^{\geq k}\right] .
$$

By construction, $\succsim_{i}^{k}$ is reflexive and transitive. The relation $\succsim_{i}^{R}$ always refines $\succsim_{i}^{k}$. $R$-linearity is equivalent to saying that each $\succsim_{i}^{k}$ is complete and that these relations are compatible, i.e., that $\succsim_{i}^{R}=\bigcap_{k \in R^{+}} \succsim_{i}^{k}$ is complete.

On top of the fact that all relations $\succsim_{i}^{R}$ can have at most $r$ distinct equivalence classes, the noncompensatory sorting model also implies that all relations $\succsim_{i}^{k}$ can have at most 2 distinct equivalence classes. This is the key to the following condition.

We say that $\left\langle C^{k}\right\rangle_{k \in R}$ is $R$-2-graded on attribute $i \in N$ (condition 2-graded ${ }_{i}^{R}$ ) if:

$$
\left.\begin{array}{l}
\left(x_{i}, a_{-i}\right) \in C^{\geq k} \\
\text { and } \\
\left(y_{i}, a_{-i}\right) \in C^{\geq k} \\
\text { and } \\
\left(y_{i}, b_{-i}\right) \in C^{\geq \ell}
\end{array}\right\} \Rightarrow\left\{\begin{array}{c}
\left(x_{i}, b_{-i}\right) \in C^{\geq \ell} \\
\text { or } \\
\left(z_{i}, a_{-i}\right) \in C^{\geq k}
\end{array} \quad\left(2 \text { graded }_{i}^{R}\right)\right.
$$


for all $k, \ell \in R^{+}$with $\ell \leq k$, all $x_{i}, y_{i}, z_{i} \in X_{i}$, and all $a_{-i}, b_{-i} \in X_{-i}$. We say that $\left\langle C^{k}\right\rangle_{k \in R}$ is $R$-2-graded if it is $R$-2-graded on all $i \in N$. Condition 2-graded ${ }_{i}^{R}$ generalizes condition 2-graded $i$ introduced in Bouyssou and Marchant (2005). A similar idea has been used, for models using binary relations, in Bouyssou and Pirlot (2002a, 2005) and Greco et al. (2001a).

It is easy to see that the violation of condition 2 -graded ${ }_{i}^{R}$ will imply that some relation $\succsim_{i}^{k}$ has more than 2 distinct equivalence classes. Indeed, suppose, in contradiction with condition $2-\operatorname{graded}_{i}^{R}$, that $\left(x_{i}, a_{-i}\right) \in C^{\geq k}$ and $\left(y_{i}, b_{-i}\right) \in C^{\geq k}$, while $\left(x_{i}, b_{-i}\right) \in C^{<k}$ and $\left(z_{i}, a_{-i}\right) \in C^{<k}$. This implies $y_{i} \succ_{i}^{k} x_{i}$ and $x_{i} \succ_{i}^{k} z_{i}$.

As shown below, $R$-2-gradedness is necessary for the noncompensatory sorting model.

\section{Lemma 17}

If $\left\langle C^{k}\right\rangle_{k \in R}$ has a representation in the noncompensatory sorting model then it is R-2-graded.

\section{PROOF}

Suppose that condition 2-graded ${ }_{i}^{R}$ is violated so that, for some $\ell, k \in R^{+}$with $\ell \leq k,\left(x_{i}, a_{-i}\right) \in C^{\geq k},\left(y_{i}, a_{-i}\right) \in C^{\geq k},\left(y_{i}, b_{-i}\right) \in C^{\geq \ell},\left(x_{i}, b_{-i}\right) \in C^{<\ell}$ and $\left(z_{i}, a_{-i}\right) \in C^{<k}$. In the noncompensatory sorting model, $\left(y_{i}, b_{-i}\right) \in C^{\geq \ell}$ and $\left(x_{i}, b_{-i}\right) \in C^{<\ell}$ imply $x_{i} \notin \mathscr{A}_{i}^{\ell}$. Similarly, $\left(x_{i}, a_{-i}\right) \in C^{\geq k}$ and $\left(z_{i}, a_{-i}\right) \in C^{<k}$ imply $x_{i} \in \mathscr{A}_{i}^{k}$. This is contradictory since $\mathscr{A}_{i}^{k} \subseteq \mathscr{A}_{i}^{\ell}$. Hence, condition 2 -graded ${ }_{i}^{R}$ holds.

\section{Remark 18}

It is easy to see that the stronger condition obtained from 2-graded ${ }_{i}^{R}$ by omitting the premise $\left(y_{i}, a_{-i}\right) \in C^{\geq k}$ is also necessary for the noncompensatory sorting model. The role of this additional premise is to ensure that condition 2 -graded ${ }_{i}^{R}$ is independent from condition $\operatorname{linear}_{i}^{R}$.

We note below that the conjunction of $2 \operatorname{graded}_{i}^{R}$ and $\operatorname{linear}_{i}^{R}$ is exactly equivalent to this stronger condition that has been used in Słowiński et al. (2002) for the study of a sorting model based on a Sugeno integral. We will study this model later. For the moment, let us simply note the following:

\section{Lemma 19}

Let $\left\langle C^{k}\right\rangle_{k \in R}$ be a partition of $X$. This partition satisfies 2-graded ${ }_{i}^{R}$ and linear $_{i}^{R}$ iff it satisfies

$$
\left.\begin{array}{c}
\left(x_{i}, a_{-i}\right) \in C^{\geq k} \\
\text { and } \\
\left(y_{i}, b_{-i}\right) \in C^{\geq \ell}
\end{array}\right\} \Rightarrow\left\{\begin{array}{c}
\left(x_{i}, b_{-i}\right) \in C^{\geq \ell} \\
\text { or } \\
\left(z_{i}, a_{-i}\right) \in C^{\geq k}
\end{array}\right.
$$

for all $k, \ell \in R^{+}$with $\ell \leq k$, all $x_{i}, y_{i}, z_{i} \in X_{i}$, and all $a_{-i}, b_{-i} \in X_{-i}$. 


\section{PROOF}

It is clear that (13) implies 2 -graded ${ }_{i}^{R}$. Taking $z_{i}=y_{i}$ shows that it also implies linear $_{i}^{R}$. Let us show that the converse implication also holds.

Suppose that $\left(x_{i}, a_{-i}\right) \in C^{\geq k}$ and $\left(y_{i}, b_{-i}\right) \in C^{\geq \ell}$. If $\left(y_{i}, a_{-i}\right) \in C^{\geq k}$, 2 graded $_{i}^{R}$ implies the desired conclusion. If $\left(y_{i}, a_{-i}\right) \notin C^{\geq k},\left(x_{i}, a_{-i}\right) \in C^{\geq k}$ and $\left(y_{i}, b_{-i}\right) \in C^{\geq \ell}$ imply, using linear ${ }_{i}^{R},\left(x_{i}, b_{-i}\right) \in C^{\geq \ell}$.

The following lemma makes clear the consequences of conditions $\operatorname{linear}_{i}^{R}$ and 2 -graded ${ }_{i}^{R}$ using the relations $\succsim_{i}^{k}$.

\section{Lemma 20}

Conditions linear ${ }_{i}^{R}$ and 2-graded ${ }_{i}^{R}$ hold iff the following three conditions hold:

(a) $\succsim_{i}^{k}$ is a weak order having at most two distinct equivalence classes,

(b) $\left[x_{i} \succ_{i}^{k} y_{i}\right] \Rightarrow\left[x_{i} \succsim_{i}^{\ell} y_{i}\right.$, for all $\left.\ell \in R^{+}\right]$,

(c) $\left[x_{i} \sim_{i}^{k} z_{i}\right.$ and $\left.x_{i} \succ_{i}^{k} y_{i}\right] \Rightarrow\left[x_{i} \sim_{i}^{\ell} z_{i}\right.$, for all $\ell \in R^{+}$such that $\left.\ell<k\right]$.

for all $k \in R^{+}$and all $x_{i}, y_{i}, z_{i} \in X_{i}$.

PROOF

Part $[\Leftarrow]$. Suppose first that $\operatorname{linear}_{i}^{R}$ is violated so that, for some $k, \ell \in R^{+}$, some $x_{i}, y_{i} \in X_{i}$ and some $a_{-i}, b_{-i} \in X_{-i},\left(x_{i}, a_{-i}\right) \in C^{k},\left(y_{i}, b_{-i}\right) \in C^{\ell}$, $\left(y_{i}, a_{-i}\right) \in C^{<k}$ and $\left(x_{i}, b_{-i}\right) \in C^{<\ell}$. Using (a), we know that $\succsim_{i}^{k}$ and $\succsim_{i}^{\ell}$ are weak orders. Hence, we have $x_{i} \succ_{i}^{k} y_{i}$ and $y_{i} \succ_{i}^{\ell} x_{i}$, contradicting (b).

Suppose now that 2 -graded ${ }_{i}^{R}$ is violated so that, for some $k, \ell \in R^{+}$such that $\ell \leq k,\left(x_{i}, a_{-i}\right) \in C^{\geq k},\left(y_{i}, a_{-i}\right) \in C^{\geq k},\left(y_{i}, b_{-i}\right) \in C^{\geq \ell},\left(x_{i}, b_{-i}\right) \in C^{<\ell}$ and $\left(z_{i}, a_{-i}\right) \in C^{<k}$. Using (a), this implies $x_{i} \succ_{i}^{k} z_{i}, y_{i} \succ_{i}^{k} z_{i}$ and $y_{i} \succ_{i}^{\ell} x_{i}$. If $k=\ell$, we obtain $y_{i} \succ_{i}^{k} x_{i}$ and $x_{i} \succ_{i}^{k} z_{i}$, which implies that $\succsim_{i}^{k}$ has three distinct equivalence classes, violating (a). Suppose henceforth that $k>\ell$. Using (b), $y_{i} \succ_{i}^{\ell} x_{i}$ implies $y_{i} \succsim_{i}^{k} x_{i}$. Suppose first that $y_{i} \sim_{i}^{k} x_{i}$. Using (c), $x_{i} \sim_{i}^{k} y_{i}$ and $x_{i} \succ_{i}^{k} z_{i}$ imply $x_{i} \sim_{i}^{\ell} y_{i}$, a contradiction. Suppose now that $y_{i} \succ_{i}^{k} x_{i}$. Since $x_{i} \succ_{i}^{k} z_{i}$, this would imply that $\succsim_{i}^{k}$ has three distinct equivalence classes, in contradiction with (a).

Part $[\Rightarrow]$. Using linear ${ }_{i}^{R}$, we know that $\succsim_{i}^{R}$ is complete. Since $\succsim_{i}^{R}$ refines $\succsim_{i}^{k}$, it follows that $\succsim_{i}^{k}$ is complete and, hence, a weak order. Clearly, $x_{i} \succ_{i}^{k} y_{i}$ and $y_{i} \succ_{i}^{\ell} x_{i}$ would violate linear ${ }_{i}^{R}$. Hence, (b) holds.

Suppose that, for some $k \in R^{+}, \succsim_{i}^{k}$ has at least three equivalence classes so that, for some $x_{i}, y_{i}, z_{i} \in X_{i}$, we have $x_{i} \succ_{i}^{k} y_{i}$ and $y_{i} \succ_{i}^{k} z_{i}$. Using the definition of $\succsim_{i}^{k}$, we have, for some $a_{-i}, b_{-i} \in X_{-i},\left(x_{i}, a_{-i}\right) \in C^{k},\left(y_{i}, a_{-i}\right) \in$ $C^{<k},\left(y_{i}, b_{-i}\right) \in C^{k},\left(z_{i}, b_{-i}\right) \in C^{<k}$. Using linear ${ }_{i}^{R},\left(x_{i}, a_{-i}\right) \in C^{k},\left(y_{i}, b_{-i}\right) \in$ $C^{k}$ and $\left(y_{i}, a_{-i}\right) \in C^{<k}$ imply $\left(x_{i}, b_{-i}\right) \in C^{\geq k}$. Using 2-graded ${ }_{i}^{R}$ with $\ell=k$, 
$\left(y_{i}, b_{-i}\right) \in C^{k},\left(x_{i}, b_{-i}\right) \in C^{\geq k}$ and $\left(x_{i}, a_{-i}\right) \in C^{k}$ imply, either $\left(y_{i}, a_{-i}\right) \in C^{\geq k}$ or $\left(z_{i}, b_{-i}\right) \in C^{\geq k}$, a contradiction. Hence, (a) holds.

Suppose now that, for some $k, \ell \in R^{+}$such that $\ell<k$ and some $x_{i}, y_{i}, z_{i} \in$ $X_{i}, x_{i} \sim_{i}^{k} z_{i}, x_{i} \succ_{i}^{k} y_{i}$ and $\operatorname{Not}\left[x_{i} \sim_{i}^{\ell} z_{i}\right]$. Suppose that $x_{i} \succ_{i}^{\ell} z_{i}$, the proof for the other case being similar. By definition, $x_{i} \sim_{i}^{k} z_{i}$ and $x_{i} \succ_{i}^{k} y_{i}$ imply that $\left(x_{i}, a_{-i}\right) \in C^{k},\left(z_{i}, a_{-i}\right) \in C^{k}$ and $\left(y_{i}, a_{-i}\right) \in C^{<k}$, for some $a_{-i} \in X_{-i}$. Similarly $x_{i} \succ_{i}^{\ell} z_{i}$ implies $\left(x_{i}, b_{-i}\right) \in C^{\ell}$ and $\left(z_{i}, b_{-i}\right) \in C^{<\ell}$, for some $b_{-i} \in$ $X_{-i}$. Using 2-graded ${ }_{i}^{R},\left(z_{i}, a_{-i}\right) \in C^{k},\left(x_{i}, a_{-i}\right) \in C^{k}$ and $\left(x_{i}, b_{-i}\right) \in C^{\ell}$ imply $\left(z_{i}, b_{-i}\right) \in C^{\geq \ell}$ or $\left(y_{i}, a_{-i}\right) \in C^{\geq k}$, a contradiction. This shows that (c) holds, which completes the proof.

\subsection{Background on twofold partitions}

If a partition $\left\langle C^{k}\right\rangle_{k \in R}$ has a representation in the noncompensatory sorting model, all the twofold partitions $\left\langle C^{\geq k}, C^{<k}\right\rangle$ will have a representation in the noncompensatory sorting model. We briefly recall below the main points of the analysis of twofold partitions as given in Bouyssou and Marchant (2005).

Consider a twofold partition $\langle\mathscr{A}, \mathscr{U}\rangle$ of $X$. We define on each $X_{i}$ the binary relation $\succsim_{i}$ letting, for all $x_{i}, y_{i} \in X_{i}$,

$$
x_{i} \succsim_{i} y_{i} \Leftrightarrow\left[\text { for all } a_{-i} \in X_{-i},\left(y_{i}, a_{-i}\right) \in \mathscr{A} \Rightarrow\left(x_{i}, a_{-i}\right) \in \mathscr{A}\right] .
$$

In Bouyssou and Marchant (2005), we prove the following:

\section{Proposition 21}

Let $\langle\mathscr{A}, \mathscr{U}\rangle$ be a twofold partition $X$. There are subsets $\mathscr{B}_{i} \subseteq X_{i}$ and a subset $\mathscr{G} \subseteq 2^{N}$ that is such that, for all $I, J \in 2^{N}$,

$$
[I \in \mathscr{G} \text { and } I \subseteq J] \Rightarrow J \in \mathscr{G},
$$

such that, for all $x \in X$,

$$
x \in \mathscr{A} \Leftrightarrow\left\{i \in N: x_{i} \in \mathscr{B}_{i}\right\} \in \mathscr{G},
$$

iff

$$
\left.\begin{array}{c}
\left(x_{i}, a_{-i}\right) \in \mathscr{A} \\
\text { and } \\
\left(y_{i}, b_{-i}\right) \in \mathscr{A}
\end{array}\right\} \Rightarrow\left\{\begin{array}{c}
\left(y_{i}, a_{-i}\right) \in \mathscr{A} \\
\text { or } \\
\left(x_{i}, b_{-i}\right) \in \mathscr{A}
\end{array}\right.
$$

and

$$
\left.\begin{array}{l}
\left(x_{i}, a_{-i}\right) \in \mathscr{A} \\
\text { and } \\
\left(y_{i}, a_{-i}\right) \in \mathscr{A} \\
\text { and } \\
\left(y_{i}, b_{-i}\right) \in \mathscr{A}
\end{array}\right\} \Rightarrow\left\{\begin{array}{c}
\left(x_{i}, b_{-i}\right) \in \mathscr{A} \\
\text { or } \\
\left(z_{i}, a_{-i}\right) \in \mathscr{A}
\end{array}\right.
$$


for all $i \in N$, all $x_{i}, y_{i}, z_{i} \in X_{i}$ and all $a_{-i}, b_{-i} \in X_{-i}$. Furthermore:

1. Conditions (16) and (17) are independent.

2. The representation $\left\langle\mathscr{G},\left\langle\mathscr{B}_{i}\right\rangle_{i \in N}\right\rangle$ of $\langle\mathscr{A}, \mathscr{U}\rangle$ is unique iff all attributes are influent for $\langle\mathscr{A}, \mathscr{U}\rangle$.

3. Suppose that $i \in N$ is influent for $\langle\mathscr{A}, \mathscr{U}\rangle$. In all representations $\left\langle\mathscr{G},\left\langle\mathscr{B}_{i}\right\rangle\right\rangle$ of $\langle\mathscr{A}, \mathscr{U}\rangle, \mathscr{B}_{i}$ must coincide with the first equivalence class of $\succsim_{i}$.

4. If $j \in N$ is degenerate for $\langle\mathscr{A}, \mathscr{U}\rangle$, it is always possible to take $\mathscr{B}_{j}=\varnothing$. With such a choice, we may always choose $\mathscr{G}$ in such a way that $I \in \mathscr{G}$ whenever there is some $x \in \mathscr{A}$ such that $\left\{i \in N: x_{i} \in \mathscr{B}_{i}\right\} \subseteq I$.

5. Furthermore, keeping the set $\mathscr{G}$ as above, on each degenerate attribute $j \in N$ we may modify $\mathscr{B}_{j}$ taking it to be an arbitrary subset of $X_{j}$. If this subset is taken to be strict, after this modification, we still have that $I \in \mathscr{G}$ whenever there is some $x \in \mathscr{A}$ such that $\left\{i \in N: x_{i} \in \mathscr{B}_{i}\right\} \subseteq I$.

Taking $\mathscr{A}=C^{\geq k}$ shows that, if a partition $\left\langle C^{k}\right\rangle_{k \in R}$ is $R$-linear, then all twofold partitions $\left\langle C^{\geq k}, C^{<k}\right\rangle$ will satisfy (16). Similarly, still taking $\mathscr{A}=$ $C^{\geq k}$, if $\left\langle C^{k}\right\rangle_{k \in R}$ is $R$-2-graded, then all twofold partitions $\left\langle C^{\geq k}, C^{<k}\right\rangle$ will satisfy (17). Hence, if $\left\langle C^{k}\right\rangle_{k \in R}$ is $R$-linear and $R$-2-graded, all twofold partitions $\left\langle C^{\geq k}, C^{<k}\right\rangle$ will have a representation in the noncompensatory sorting model. These representations of the twofold partitions $\left\langle C^{\geq k}, C^{<k}\right\rangle$ will form the basis of our analysis.

\subsection{Result}

Our main result in this section says that $R$-linearity and $R$-2-gradedness characterize the noncompensatory sorting model.

\section{Theorem 22}

An r-fold partition $\left\langle C^{k}\right\rangle_{k \in R}$ of $X$ has a representation in the noncompensatory sorting model iff it is $R$-linear and $R$-2-graded.

\section{ProOF}

Necessity results from Lemmas 16 and 17 . We show sufficiency. Because $\left\langle C^{k}\right\rangle_{k \in R}$ is a partition, it is clear that, for all $k \in R^{+},\left\langle C^{\geq k}, C^{<k}\right\rangle$ is a partition, so that there is at least one attribute that is influent for $\left\langle C^{\geq k}, C^{<k}\right\rangle$. Since $\left\langle C^{k}\right\rangle_{k \in R}$ is $R$-linear and $R$-2-graded, for all $k \in R^{+}$, the twofold partition $\left\langle C^{\geq k}, C^{<k}\right\rangle$ satisfies (16) and (17). Using Proposition 21, there are 
subsets $\mathscr{B}_{i}^{k} \subseteq X_{i}$ and a subset $\mathscr{G}^{k} \subseteq 2^{N}$ satisfying (14) such that, for all $x \in X$,

$$
x \in C^{\geq k} \Leftrightarrow\left\{i \in N: x_{i} \in \mathscr{B}_{i}^{k}\right\} \in \mathscr{G}^{k}
$$

We define below $\mathscr{F}^{k}$ and $\mathscr{A}_{i}^{k}$ on the basis of $\mathscr{G}^{k}$ and $\mathscr{B}_{i}^{k}$ distinguishing two cases.

Case $k=r$

Let $\left\langle\mathscr{G}^{r},\left\langle\mathscr{B}_{i}^{r}\right\rangle_{i \in N}\right\rangle$ be the representation of $\left\langle C^{\geq r}, C^{<r}\right\rangle$ derived from Proposition 21. It is such that if $i \in N$ is degenerate for $\left\langle C^{\geq r}, C^{<r}\right\rangle$, then $\mathscr{B}_{i}^{r}=\varnothing$. Take $\mathscr{F}^{r}=\mathscr{G}^{r}$ and, for all $i \in N, \mathscr{A}_{i}^{r}=\mathscr{B}_{i}^{r}$. By construction, $\left\langle\mathscr{F}^{r},\left\langle\mathscr{A}_{i}^{r}\right\rangle_{i \in N}\right\rangle$ is a representation of $\left\langle C^{\geq r}, C^{<r}\right\rangle$ in the noncompensatory sorting model.

Case $k<r$

For $k=r-1, r-2, \ldots, 2$, let $\left\langle\mathscr{G}^{k},\left\langle\mathscr{B}_{i}^{k}\right\rangle_{i \in N}\right\rangle$ be the representation of $\left\langle C^{\geq k}, C^{<k}\right\rangle$ derived from Proposition 21. We build $\mathscr{F}^{k}$ and $\mathscr{A}_{i}^{k}$ in sequence, starting with $k=r-1$.

If $i \in N$ is influent for $\left\langle C^{\geq k}, C^{<k}\right\rangle$, take $\mathscr{A}_{i}^{k}=\mathscr{B}_{i}^{k}$. If $i \in N$ is degenerate for $\left\langle C^{\geq k}, C^{<k}\right\rangle$, we have $\mathscr{B}_{i}^{k}=\varnothing$. In such a case, we take $\mathscr{A}_{i}^{k}=\mathscr{A}_{i}^{k+1}$. In either case, $\mathscr{A}_{i}^{k}$ is a strict subset of $X_{i}$. We define $\mathscr{F}^{k}$ as $\mathscr{G}^{k}$. Using parts 4 and 5 of Proposition 21, we know that $\left\langle\mathscr{F}^{k},\left\langle\mathscr{A}_{i}^{k}\right\rangle_{i \in N}\right\rangle$ is a representation of $\left\langle C^{\geq k}, C^{<k}\right\rangle$ in the noncompensatory sorting model and we have $I \in \mathscr{F}^{k}$ whenever there is some $x \in C^{\geq k}$ such that $\left\{i \in N: x_{i} \in \mathscr{A}_{i}^{k}\right\} \subseteq I$.

Proof that $\mathscr{A}_{i}^{k} \subseteq \mathscr{A}_{i}^{k-1}$

Let us prove that, for all $i \in N$ and all $k \in\{3,4, \ldots, r\}$ we have $\mathscr{A}_{i}^{k} \subseteq \mathscr{A}_{i}^{k-1}$. If attribute $i \in N$ is not influent for $\left\langle C^{\geq \ell}, C^{<\ell}\right\rangle$ for $\ell=r, r-1, \ldots, k$, we have $\mathscr{A}_{i}^{k}=\varnothing$ and there is nothing to prove. Similarly if $i \in N$ is not influent for $\left\langle C^{\geq k-1}, C^{<k-1}\right\rangle$, we have $\mathscr{A}_{i}^{k-1}=\mathscr{A}_{i}^{k}$ and there is nothing to prove either. Suppose henceforth that $i \in N$ is influent for $\left\langle C^{\geq k-1}, C^{<k-1}\right\rangle$ and let $\ell$ be the smallest element in $\{r, r-1, \ldots, k\}$ such that $i$ is influent for $\left\langle C^{\geq \ell}, C^{<\ell}\right\rangle$. By construction, we have $\mathscr{A}_{i}^{k}=\mathscr{A}_{i}^{\ell}$. Suppose, in contradiction with the thesis, that $x_{i} \in \mathscr{A}_{i}^{\ell}$ and $x_{i} \notin \mathscr{A}_{i}^{k-1}$ with $\ell \geq k$.

Since $i \in N$ is influent for $\left\langle C^{\geq \ell}, C^{<\ell}\right\rangle$ and $x_{i} \in \mathscr{A}_{i}^{\ell}$, we know that $\left(x_{i}, a_{-i}\right) \in C^{\geq \ell}$ and $\left(y_{i}, a_{-i}\right) \notin C^{\geq \ell}$, for some $y_{i} \in X_{i}$ and some $a_{-i} \in X_{-i}$. Similarly, since $i \in N$ is influent for $\left\langle C^{\geq k-1}, C^{<k-1}\right\rangle$ and $x_{i} \notin \mathscr{A}_{i}^{k-1}$, we know that $\left(z_{i}, b_{-i}\right) \in C^{\geq k-1}$ and $\left(x_{i}, b_{-i}\right) \notin C^{\geq k-1}$, for some $z_{i} \in X_{i}$ and some $b_{-i} \in X_{-i}$.

Using linear ${ }_{i}^{R},\left(x_{i}, a_{-i}\right) \in C^{\geq \ell},\left(z_{i}, b_{-i}\right) \in C^{\geq k-1}$ and $\left(x_{i}, b_{-i}\right) \notin C^{\geq k-1}$ imply $\left(z_{i}, a_{-i}\right) \in C^{\geq \ell}$. Using 2 -graded ${ }_{i}^{R},\left(x_{i}, a_{-i}\right) \in C^{\geq \ell},\left(z_{i}, a_{-i}\right) \in C^{\geq \ell}$ and 
$\left(z_{i}, b_{-i}\right) \in C^{\geq k-1}$ imply $\left(x_{i}, b_{-i}\right) \in C^{\geq k-1}$ or $\left(y_{i}, a_{-i}\right) \in C^{\geq \ell}$, a contradiction.

Proof that $\mathscr{F}^{k} \subseteq \mathscr{F}^{k-1}$

Let us now prove that $\mathscr{F}^{k} \subseteq \mathscr{F}^{k-1}$. By construction, we know that, for all $k \in\{3,4, \ldots, r\}, I \in \mathscr{F}^{k}$ whenever there is some $x \in C^{\geq k}$ such that $A^{k}(x)=\left\{i \in N: x_{i} \in \mathscr{A}_{i}^{k}\right\} \subseteq I$. Let $I \in \mathscr{F}^{k}$ and let $x \in X$ be such that $x \in C^{\geq k}$ and $A^{k}(x) \subseteq I$. Starting with such an alternative $x \in X$, let us build an alternative $x^{\prime} \in X$ as follows. For all $i \in N$ such that $x_{i} \in \mathscr{A}_{i}^{k}$, let $x_{i}^{\prime}=x_{i}$. Because $\mathscr{A}_{i}^{k} \subseteq \mathscr{A}_{i}^{k-1}$, we know that on these attributes $x_{i}^{\prime} \in \mathscr{A}_{i}^{k-1}$. For all $i \in N$ such that $x_{i} \notin \mathscr{A}_{i}^{k}$, we consider two cases:

1. if $i$ is influent for $\left\langle C^{\geq k-1}, C^{<k-1}\right\rangle$, by construction there is a $z_{i} \in X_{i}$ such that $z_{i} \notin \mathscr{A}_{i}^{k-1}$. In this case, let $x_{i}^{\prime}=z_{i}$, so that $x_{i}^{\prime} \notin \mathscr{A}_{i}^{k-1}$.

2. If $i$ is not influent for $\left\langle C^{\geq k-1}, C^{<k-1}\right\rangle$, we have $\mathscr{A}_{i}^{k}=\mathscr{A}_{i}^{k-1}$. In this case, take $x_{i}^{\prime}$ equal to $x_{i}$, so that $x_{i}^{\prime} \notin \mathscr{A}_{i}^{k-1}$.

By construction, we have $A^{k}(x)=A^{k}\left(x^{\prime}\right)=A^{k-1}\left(x^{\prime}\right) \subseteq I$. Because $A^{k}(x)=$ $A^{k}\left(x^{\prime}\right)$ and $x \in C^{\geq k}$, we know that $x^{\prime} \in C^{\geq k}$ so that $x^{\prime} \in C^{\geq k-1}$. Since $x \in C^{\geq k-1}$ and $A^{k-1}\left(x^{\prime}\right) \subseteq I$, we have $I \in \mathscr{F}^{k-1}$. This completes the proof.

The construction of the representation in the noncompensatory sorting model is illustrated below.

\section{Example 23}

Suppose that $n=3, X_{1}=X_{2}=X_{3}=\{9,10,11\}$. We consider a threefold partition $\left\langle C^{1}, C^{2}, C^{3}\right\rangle$ such that $C^{3}=\{(9,10,10),(9,10,11),(9,11,10)$, $(9,11,11),(10,9,10),(10,9,11),(10,10,9),(10,10,10),(10,10,11),(10,11,9)$, $(10,11,10),(10,11,11),(11,9,10),(11,9,11),(11,10,9),(11,10,10),(11,10,11)$, $(11,11,9),(11,11,10),(11,11,11)\}, C^{2}=\{(9,10,9),(9,11,9),(10,9,9)$, $(11,9,9)\}$ and $C^{1}=\{(9,9,9),(9,9,10),(9,9,11)\}$.

This partition can be obtained with the pessimistic version of ELECTRE TRI with $(10,10,10)$ as the limiting profile between $C^{3}$ and $C^{2}$ and $(10,10,9)$ as the limiting profile between $C^{2}$ and $C^{1}, S_{i}=\geq$ and $V_{i}=\varnothing$ for all $i \in N$, $w_{1}=w_{2}=w_{3}=1 / 3, \lambda=2 / 3$. This shows that it is $R$-linear and $R$-2-graded.

All attributes are influent for the twofold partition $\left\langle C^{\geq 3}, C^{<3}\right\rangle$. Let $\left\langle\mathscr{G}^{3}\right.$, $\left.\left\langle\mathscr{B}_{1}^{3}, \mathscr{B}_{2}^{3}, \mathscr{B}_{3}^{3}\right\rangle\right\rangle$ be the unique representation of $\left\langle C^{\geq 3}, C^{<3}\right\rangle$ derived from Proposition 21. We have: $\mathscr{B}_{1}^{3}=\mathscr{B}_{2}^{3}=\mathscr{B}_{3}^{3}=\{10,11\}$ and $\mathscr{G}^{3}=\{\{1,2\},\{1,3\}$, $\{2,3\},\{1,2,3\}\}$. We take $\mathscr{A}_{i}^{3}=\mathscr{B}_{i}^{3}$, for all $i \in N$, and $\mathscr{F}^{3}=\mathscr{G}^{3}$.

Only attributes 1 and 2 are influent for the twofold partition $\left\langle C^{\geq 2}, C^{<2}\right\rangle$. The representation $\left\langle\mathscr{G}^{2},\left\langle\mathscr{B}_{1}^{2}, \mathscr{B}_{2}^{2}, \mathscr{B}_{3}^{2}\right\rangle\right\rangle$ of $\left\langle C^{\geq 2}, C^{<2}\right\rangle$ derived from Proposition 21 is such that $\mathscr{B}_{1}^{2}=\mathscr{B}_{2}^{2}=\{10,11\}, \mathscr{B}_{3}^{2}=\varnothing$ and $\mathscr{G}^{2}=\{\{1\},\{2\}$, 
$\{1,2\},\{1,3\},\{2,3\},\{1,2,3\}\}$. As explained in the proof of Theorem 22, we take $\mathscr{A}_{1}^{2}=\mathscr{B}_{1}^{2}, \mathscr{A}_{2}^{2}=\mathscr{B}_{2}^{2}, \mathscr{A}_{3}^{2}=\mathscr{A}_{3}^{3}$ and $\mathscr{F}^{2}=\mathscr{G}^{2}$.

It can easily be checked that $\left\langle\mathscr{F}^{k},\left\langle\mathscr{A}_{i}^{k}\right\rangle_{i \in N}\right\rangle$ is a representation of $\left\langle C^{k}\right\rangle_{k \in R}$ in the noncompensatory sorting model. This is detailed in Table 1.

\subsection{The noncompensatory sorting model and the Sugeno integral}

Salvatore Greco, Benedetto Matarazzo and Roman Słowiński have brought to our attention the fact that Theorem 22 has very close connections with Theorem 2.4 given, without proof, in Słowiński et al. (2002) (our results have been obtained independently). The purpose of Słowiński et al. (2002, Theorem 2.4) is to characterize partitions that can be represented using a Sugeno integral.

Let $\left\langle C^{k}\right\rangle_{k \in R}$ be a partition of $X$. We say that $\left\langle C^{k}\right\rangle_{k \in R}$ can be represented using a Sugeno integral, if there are:

- a non-negative real valued function $f_{i}$ on $X_{i}$, for all $i \in N$,

- $r-1$ real numbers such that $0<\rho_{2}<\rho_{3}<\ldots<\rho_{r}$

- a real valued function ${ }^{5} \mu$ on $2^{N}$ that is nondecreasing w.r.t. inclusion (i.e., such that $A \subseteq B$ implies $\mu(A) \leq \mu(B)$ ) and such that $\mu(\varnothing)=0$,

such that, for all $x \in X$,

$$
x \in C^{\geq k} \Leftrightarrow S_{\left\langle\mu, f_{i}\right\rangle}(x)=\bigvee_{I \subseteq N}\left[\bigwedge_{i \in I}\left[f_{i}\left(x_{i}\right)\right] ; \mu(I)\right]>\rho_{k},
$$

where $S_{\left\langle\mu, f_{i}\right\rangle}(x)$ is called the (discrete) Sugeno integral of the vector $\left(f_{1}\left(x_{1}\right)\right.$, $\left.f_{2}\left(x_{2}\right), \ldots, f_{n}\left(x_{n}\right)\right)$ w.r.t. the capacity $\mu$. We refer the reader to Marichal (2000) for a detailed study of various equivalent forms of $S_{\left\langle\mu, f_{i}\right\rangle}(x)$, including the more common forms that involve a reordering of the vector $\left(f_{1}\left(x_{1}\right), f_{2}\left(x_{2}\right)\right.$, $\left.\ldots, f_{n}\left(x_{n}\right)\right)$.

As already observed in Bouyssou and Marchant (2005) for the particular case of two categories, a partition can be represented in the noncompensatory sorting model iff it can be represented using a Sugeno integral.

Observe first that any partition that can be represented in the noncompensatory sorting model has a representation using a Sugeno integral. Take

\footnotetext{
${ }^{5}$ The function $\mu$ is usually called a capacity. It is often supposed that the capacity is normalized so that $\mu(N)=1$. This is not necessary for our purposes.
} 


\begin{tabular}{ccccc}
$x$ & Category & $B^{3}(x)$ & $B^{2}(x)$ & $A^{2}(x)$ \\
\hline$(9,10,10)$ & $C^{3}$ & $\{2,3\}$ & $\{2\}$ & $\{2,3\}$ \\
$(9,10,11)$ & $C^{3}$ & $\{2,3\}$ & $\{2\}$ & $\{2,3\}$ \\
$(9,11,10)$ & $C^{3}$ & $\{2,3\}$ & $\{2\}$ & $\{2,3\}$ \\
$(9,11,11)$ & $C^{3}$ & $\{2,3\}$ & $\{2\}$ & $\{2,3\}$ \\
$(10,9,10)$ & $C^{3}$ & $\{1,3\}$ & $\{1\}$ & $\{2,3\}$ \\
$(10,9,11)$ & $C^{3}$ & $\{1,3\}$ & $\{1\}$ & $\{2,3\}$ \\
$(10,10,9)$ & $C^{3}$ & $\{1,2\}$ & $\{1,2\}$ & $\{1,2\}$ \\
$(10,10,10)$ & $C^{3}$ & $\{1,2,3\}$ & $\{1,2\}$ & $\{1,2,3\}$ \\
$(10,10,11)$ & $C^{3}$ & $\{1,2,3\}$ & $\{1,2\}$ & $\{1,2,3\}$ \\
$(10,11,9)$ & $C^{3}$ & $\{1,2\}$ & $\{1,2\}$ & $\{1,2\}$ \\
$(10,11,10)$ & $C^{3}$ & $\{1,2,3\}$ & $\{1,2\}$ & $\{1,2,3\}$ \\
$(10,11,11)$ & $C^{3}$ & $\{1,2,3\}$ & $\{1,2\}$ & $\{1,2,3\}$ \\
$(11,9,10)$ & $C^{3}$ & $\{1,3\}$ & $\{1\}$ & $\{1,3\}$ \\
$(11,9,11)$ & $C^{3}$ & $\{1,3\}$ & $\{1\}$ & $\{1,3\}$ \\
$(11,10,9)$ & $C^{3}$ & $\{1,2\}$ & $\{1,2\}$ & $\{1,2\}$ \\
$(11,10,10)$ & $C^{3}$ & $\{1,2,3\}$ & $\{1,2\}$ & $\{1,2,3\}$ \\
$(11,10,11)$ & $C^{3}$ & $\{1,2,3\}$ & $\{1,2\}$ & $\{1,2,3\}$ \\
$(11,11,9)$ & $C^{3}$ & $\{1,2\}$ & $\{1,2\}$ & $\{1,2\}$ \\
$(11,11,10)$ & $C^{3}$ & $\{1,2,3\}$ & $\{1,2\}$ & $\{1,2,3\}$ \\
$(11,11,11)$ & $C^{3}$ & $\{1,2,3\}$ & $\{1,2\}$ & $\{1,2,3\}$ \\
$\ldots \ldots \ldots \ldots$ & $\ldots \ldots \ldots$ & $\ldots \ldots$ & $\ldots \ldots$ & $\ldots \ldots$ \\
$(9,10,9)$ & $C^{2}$ & $\{2\}$ & $\{2\}$ & $\{2\}$ \\
$(9,11,9)$ & $C^{2}$ & $\{2\}$ & $\{2\}$ & $\{2\}$ \\
$(10,9,9)$ & $C^{2}$ & $\{1\}$ & $\{1\}$ & $\{1\}$ \\
$(11,9,9)$ & $C^{2}$ & $\{1\}$ & $\{1\}$ & $\{1\}$ \\
$\ldots \ldots \ldots$ & $\ldots \ldots \ldots$ & $\ldots \ldots$ & $\ldots \ldots$ & $\ldots \ldots$ \\
$(9,9,9)$ & $C^{1}$ & $\varnothing$ & $\varnothing$ & $\varnothing$ \\
$(9,9,10)$ & $C^{1}$ & $\{3\}$ & $\varnothing$ & $\{3\}$ \\
$(9,9,11)$ & $C^{1}$ & $\{3\}$ & $\varnothing$ & $\{3\}$
\end{tabular}

Table 1: Details of Example 23

$$
\begin{gathered}
B^{3}(x)=\left\{i \in N: x_{i} \in \mathscr{B}_{i}^{3}\right\}, B^{2}(x)=\left\{i \in N: x_{i} \in \mathscr{B}_{i}^{2}\right\} \\
\mathscr{B}_{1}^{3}=\mathscr{B}_{2}^{3}=\mathscr{B}_{2}^{3}=\{10,11\}, \mathscr{A}_{1}^{3}=\mathscr{A}_{2}^{3}=\mathscr{A}_{3}^{3}=\{10,11\}, \\
\mathscr{F}^{3}=\mathscr{G}^{3}=\{\{1,2\},\{1,3\},\{2,3\},\{1,2,3\}\} \\
\mathscr{B}_{1}^{2}=\mathscr{B}_{2}^{2}=\{10,11\}, \mathscr{B}_{3}^{2}=\varnothing, \mathscr{A}_{1}^{2}=\mathscr{A}_{2}^{2}=\{10,11\}, \mathscr{A}_{3}^{2}=\{10,11\} \\
\mathscr{F}^{2}=\mathscr{G}^{2}=\{\{1\},\{2\},\{1,2\},\{1,3\},\{2,3\},\{1,2,3\}\}
\end{gathered}
$$


any numbers $\lambda_{k}$ and $\rho_{k}$ such that: $0<\lambda_{1}<\rho_{2}<\lambda_{2}<\rho_{3}<\ldots \lambda_{r-1}<\rho_{r}<$ $\lambda_{r}$. Let $\left\langle\mathscr{F}^{k},\left\langle\mathscr{A}_{i}^{k}\right\rangle_{i \in N}\right\rangle$ be a representation of $\left\langle C^{k}\right\rangle_{k \in R}$ in the noncompensatory sorting model.

For all $i \in N$, define $f_{i}$ letting, for all $x_{i} \in X_{i}$,

$$
\begin{cases}f_{i}\left(x_{i}\right)=\lambda_{r} & \text { if } x_{i} \in \mathscr{A}_{i}^{r}, \\ f_{i}\left(x_{i}\right)=\lambda_{r-1} & \text { if } x_{i} \in \mathscr{A}_{i}^{r-1} \backslash \mathscr{A}_{i}^{r}, \\ f_{i}\left(x_{i}\right)=\lambda_{r-2} & \text { if } x_{i} \in \mathscr{A}_{i}^{r-2} \backslash \mathscr{A}_{i}^{r-1}, \\ \vdots & \\ f_{i}\left(x_{i}\right)=\lambda_{2} & \text { if } x_{i} \in \mathscr{A}_{i}^{2} \backslash \mathscr{A}_{i}^{3} \\ f_{i}\left(x_{i}\right)=\lambda_{1} & \text { otherwise, }\end{cases}
$$

and $\mu$ on $2^{N}$ letting, for all $A \in 2^{N}$,

$$
\begin{cases}\mu(A)=\lambda_{r} & \text { if } A \in \mathscr{F}^{r}, \\ \mu(A)=\lambda_{r-1} & \text { if } A \in \mathscr{F}^{r-1} \backslash \mathscr{F}^{r}, \\ \mu(A)=\lambda_{r-2} & \text { if } A \in \mathscr{F}^{r-2} \backslash \mathscr{F}^{r-1}, \\ \vdots & \\ \mu(A)=\lambda_{2} & \text { if } A \in \mathscr{F}^{2} \backslash \mathscr{F}^{3}, \\ \mu(A)=\lambda_{1} & \text { otherwise. }\end{cases}
$$

With such definitions, for all $x \in X$, the value $S_{\left\langle\mu, f_{i}\right\rangle}(x)$ belongs to $\left\{\lambda_{1}, \lambda_{2}\right.$, $\left.\ldots, \lambda_{r}\right\}$. We have $x \in C^{\geq k}$ iff $\left\{i \in N: x_{i} \in \mathscr{A}_{i}^{k}\right\} \in \mathscr{F}^{k}$, which implies $S_{\left\langle\mu, f_{i}\right\rangle}(x) \geq \lambda_{k}>\rho_{k}$. Similarly, it is easy to see that if $x \notin C^{\geq k}$, we have $S_{\left\langle\mu, f_{i}\right\rangle}(x) \leq \lambda_{k-1}$, so that $S_{\left\langle\mu, f_{i}\right\rangle}(x)<\rho_{k}$. Hence, any partition that has a representation in the noncompensatory sorting model can be represented in model $(S u)$.

Consider now a partition $\left\langle C^{k}\right\rangle_{k \in R}$ of $X$ that can be represented in the model $(S u)$. Let us show that such a partition satisfies (13). Suppose that $\left(x_{i}, a_{-i}\right) \in C^{\geq k},\left(y_{i}, b_{-i}\right) \in C^{\geq \ell}$ and $\left(x_{i}, b_{-i}\right) \notin C^{\geq \ell}$. Using model $(S u)$, $\left(y_{i}, b_{-i}\right) \in C^{\geq \ell}$ and $\left(x_{i}, b_{-i}\right) \notin C^{\geq \ell}$ imply $f_{i}\left(x_{i}\right) \leq \lambda_{\ell}$. Since $\left(x_{i}, a_{-i}\right) \in C^{\geq k}$, we must have, for some $I \in 2^{N}$ such that $i \notin I, \mu(I)>\rho_{k}$ and $\bigwedge_{j \in I} f_{j}\left(a_{j}\right)>$ $\rho_{k}$. This implies $\left(z_{i}, a_{-i}\right) \in C^{\geq k}$, for all $z_{i} \in X_{i}$.

Combining the above observations and Lemma 19 with Theorem 22, we have proved:

\section{Proposition 24}

A partition $\left\langle C^{k}\right\rangle_{k \in R}$ of a set $X$ has a representation in the noncompensatory sorting model iff it has a representation in the Sugeno integral model (Su).

Słowiński et al. (2002, Theorem 2.4) state, without proof, that a partition $\left\langle C^{k}\right\rangle_{k \in R}$ can be represented in the Sugeno integral model $(S u)$ iff it satis- 
fies condition (13). The above proposition, connects Słowiński et al. (2002, Theorem 2.4) and our Theorem 22, showing, in fact, that they are characterizations of the same underlying model.

\subsection{Independence of axioms}

Let us show that none of the two conditions used in Theorem 22 can be dispensed with. Consider first the following condition:

$$
\left.\begin{array}{l}
\left(x_{i}, a_{-i}\right) \in C^{\geq k} \\
\text { and } \\
\left(y_{i}, a_{-i}\right) \in C^{\geq k} \\
\text { and } \\
\left(y_{i}, b_{-i}\right) \in C^{\geq k}
\end{array}\right\} \Rightarrow\left\{\begin{array}{c}
\left(x_{i}, b_{-i}\right) \in C^{\geq k} \\
\text { or } \\
\left(z_{i}, a_{-i}\right) \in C^{\geq k}
\end{array}\right.
$$

for all $x_{i}, y_{i}, z_{i} \in X_{i}$, all $a_{-i}, b_{-i} \in X_{-i}$ and all $k \in R^{+}$. Condition (18) is nothing but condition $2-\operatorname{graded}_{i}^{R}$ restricted to the case $\ell=k$. The following example shows that the conjunction of $R$-linearity and condition (18), for all $i \in N$, is not sufficient to precipitate the noncompensatory sorting model.

\section{Example 25}

Let $n=3, X=\left\{x_{1}, y_{1}, z_{1}\right\} \times\left\{x_{2}, y_{2}\right\} \times\left\{x_{3}, y_{3}\right\}$ and $r=3$. Let $C^{3}=$ $\left\{\left(x_{1}, x_{2}, x_{3}\right),\left(y_{1}, x_{2}, x_{3}\right)\right\}, C^{2}=\left\{\left(x_{1}, x_{2}, y_{3}\right),\left(x_{1}, y_{2}, x_{3}\right),\left(y_{1}, x_{2}, y_{3}\right),\left(y_{1}, y_{2}, x_{3}\right)\right.$, $\left.\left(y_{1}, y_{2}, y_{3}\right),\left(z_{1}, x_{2}, x_{3}\right),\left(z_{1}, x_{2}, y_{3}\right),\left(z_{1}, y_{2}, x_{3}\right)\right\}$ and $C^{1}=\left\{\left(z_{1}, y_{2}, y_{3}\right),\left(x_{1}, y_{2}, y_{3}\right)\right\}$.

We have $y_{1} \succ_{1}^{R} x_{1} \succ_{1}^{R} z_{1}, x_{2} \succ_{2}^{R} y_{2}$ and $x_{3} \succ_{3}^{R} y_{3}$, which shows that $\left\langle C^{k}\right\rangle_{k \in R}$ is $R$-linear.

The twofold partition $\left\langle C^{\geq 3}, C^{<3}\right\rangle$ has a representation in the noncompensatory sorting model with $\mathscr{B}_{1}^{3}=\left\{x_{1}, y_{1}\right\}, \mathscr{B}_{2}^{3}=\left\{x_{2}\right\}, \mathscr{B}_{3}^{3}=\left\{x_{3}\right\}$ and $\mathscr{G}^{3}=\{\{1,2,3\}\}$. This representation is unique since all attributes are influent for $\left\langle C^{\geq 3}, C^{<3}\right\rangle$. Indeed, we have $\left(x_{1}, x_{2}, x_{3}\right) \in C^{\geq 3}$ and $\left(z_{1}, x_{2}, x_{3}\right) \in C^{<3}$, $\left(x_{1}, x_{2}, x_{3}\right) \in C^{\geq 3}$ and $\left(x_{1}, y_{2}, x_{3}\right) \in C^{<3},\left(x_{1}, x_{2}, x_{3}\right) \in C^{\geq 3}$ and $\left(x_{1}, x_{2}, y_{3}\right) \in$ $C^{<3}$.

Similarly, the twofold partition $\left\langle C^{\geq 2}, C^{<2}\right\rangle$ has a representation in the noncompensatory sorting model with $\mathscr{B}_{1}^{1}=\left\{y_{1}\right\}, \mathscr{B}_{2}^{2}=\left\{x_{2}\right\}, \mathscr{B}_{3}^{2}=\left\{x_{3}\right\}$ and $\mathscr{G}^{2}=2^{N} \backslash\{\varnothing\}$. This representation is unique since all attributes are influent for $\left\langle C^{\geq 2}, C^{<2}\right\rangle$. Indeed, we have $\left(y_{1}, y_{2}, y_{3}\right) \in C^{\geq 2}$ and $\left(x_{1}, y_{2}, y_{3}\right) \in C^{<2}$, $\left(x_{1}, x_{2}, y_{3}\right) \in C^{\geq 2}$ and $\left(x_{1}, y_{2}, y_{3}\right) \in C^{<2},\left(x_{1}, y_{2}, x_{3}\right) \in C^{\geq 2}$ and $\left(x_{1}, y_{2}, y_{3}\right) \in$ $C^{<2}$.

Since each of the twofold partitions induced by $\left\langle C^{1}, C^{2}, C^{3}\right\rangle$ has a representation in the noncompensatory sorting model, condition (18) holds, for all $i \in N$. However the partition $\left\langle C^{1}, C^{2}, C^{3}\right\rangle$ cannot be represented in the noncompensatory sorting model. Indeed, $\left(x_{1}, x_{2}, x_{3}\right) \in C^{\geq 3}$ and 
$\left(z_{1}, x_{2}, x_{3}\right) \in C^{<3}$ would imply $x_{1} \in \mathscr{A}_{i}^{3}$. Similarly, $\left(y_{1}, y_{2}, y_{3}\right) \in C^{\geq 2}$ and $\left(x_{1}, y_{2}, y_{3}\right) \in C^{<2}$ would imply $x_{1} \notin \mathscr{A}_{i}^{2}$, violating $\mathscr{A}_{i}^{3} \subseteq \mathscr{A}_{i}^{2}$.

One can check, e.g., using Lemma 20, that the partition $\left\langle C^{1}, C^{2}, C^{3}\right\rangle$ satisfies 2 -graded ${ }_{2}^{R}$ and 2 -graded $d_{3}^{R}$. Condition $2-\operatorname{graded}_{1}^{R}$ is violated since $\left(x_{1}, x_{2}, x_{3}\right) \in C^{\geq 3},\left(y_{1}, x_{2}, x_{3}\right) \in C^{\geq 3}$ and $\left(y_{1}, y_{2}, y_{3}\right) \in C^{\geq 2}$ but $\left(x_{1}, y_{2}, y_{3}\right) \notin$ $C^{\geq 2}$ and $\left(z_{1}, x_{2}, x_{3}\right) \notin C^{\geq 3}$.

Consider now the following condition

$$
\left.\begin{array}{l}
\left(x_{i}, a_{-i}\right) \in C^{k} \\
\text { and } \\
\left(y_{i}, b_{-i}\right) \in C^{k}
\end{array}\right\} \Rightarrow\left\{\begin{array}{c}
\left(y_{i}, a_{-i}\right) \in C^{\geq k} \\
\text { or } \\
\left(x_{i}, b_{-i}\right) \in C^{\geq k}
\end{array}\right.
$$

for all $x_{i}, y_{i} \in X_{i}$, all $k \in R^{+}$and all $a_{-i}, b_{-i} \in X_{-i}$. Condition (19) is nothing but condition linear ${ }_{i}^{R}$ restricted to the case $\ell=k$. It is clearly equivalent to requiring that all relations $\succsim_{i}^{k}$ are complete. The following example shows that the conjunction of $R$-2-gradedness and condition (19), for all $i \in N$, is not sufficient to precipitate the noncompensatory sorting model.

\section{Example 26}

Let $n=3, X=\left\{x_{1}, y_{1}\right\} \times\left\{x_{2}, y_{2}\right\} \times\left\{x_{3}, y_{3}\right\}$ and $r=3$. Let $C^{3}=$ $\left\{\left(x_{1}, x_{2}, x_{3}\right),\left(x_{1}, y_{2}, x_{3}\right),\left(y_{1}, y_{2}, x_{3}\right)\right\}, C^{2}=\left\{\left(y_{1}, y_{2}, y_{3}\right),\left(y_{1}, x_{2}, x_{3}\right)\right\}$ and $C^{1}=\left\{\left(x_{1}, x_{2}, y_{3}\right),\left(x_{1}, y_{2}, y_{3}\right),\left(y_{1}, x_{2}, y_{3}\right)\right\}$.

Because each $X_{i}$ has only two elements, this partition is trivially $R-2$ graded. We have $y_{2} \succ_{i}^{R} x_{2}$ and $x_{3} \succ_{i}^{R} y_{3}$, so that linear ${ }_{2}^{R}$ and linear ${ }_{3}^{R}$ hold. Since $\left(x_{1}, x_{2}, x_{3}\right) \in C^{3}$ and $\left(y_{1}, y_{2}, y_{3}\right) \in C^{2}$ but neither $\left(y_{1}, x_{2}, x_{3}\right) \in C^{\geq 3}$ nor $\left(x_{1}, y_{2}, y_{3}\right) \in C^{\geq 2}$, condition linear $_{1}^{R}$ is violated. Observe that $x_{1} \succ_{1}^{3} y_{1}$ and $y_{1} \succ_{1}^{2} x_{1}$, so that condition (19) is satisfied for attribute 1 .

\subsection{Uniqueness of the representation}

Let $\langle\mathscr{A}, \mathscr{U}\rangle$ be a twofold partition of $X$ having a representation in the noncompensatory sorting model. In Bouyssou and Marchant (2005), we prove that $\langle\mathscr{A}, \mathscr{U}\rangle$ has a unique representation in the noncompensatory sorting model if and only if all attributes are influent for $\langle\mathscr{A}, \mathscr{U}\rangle$.

Using the above observation, it is clear that if, for all $i \in N$ and all $k \in R^{+}$, attribute $i$ is influent for $\left\langle C^{\geq k}, C^{<k}\right\rangle$, the $r$-fold partition $\left\langle C^{k}\right\rangle_{k \in R}$ will have a unique representation in the noncompensatory sorting model. As shown below, the additional "nesting" constraints brought by the noncompensatory sorting model when $r>2$ are such that this sufficient condition is no longer necessary for the uniqueness of the representation. This is detailed in the following remark. 


\section{Remark 27}

Suppose that, for some $\ell \in R^{+}$, there is an attribute $j \in N$ that is degenerate for $\left\langle C^{\geq \ell}, C^{<\ell}\right\rangle$. Because, by hypothesis, $j \in N$ is influent for $\left\langle C^{k}\right\rangle_{k \in R}$, it is influent for some of the twofold partitions $\left\langle C^{\geq k}, C^{<k}\right\rangle$. Let $\tau_{j}$ be the largest $k \in R^{+}$such that $j \in N$ is influent for $\left\langle C^{\geq k}, C^{<k}\right\rangle$. Similarly, let $\beta_{j}$ be the smallest $k \in R^{+}$such that $j \in N$ is influent for $\left\langle C^{\geq k}, C^{<k}\right\rangle$. The sets $\mathscr{A}_{j}^{\tau_{j}}$ and $\mathscr{A}_{j}^{\beta_{j}}$ are nonempty strict subsets of $X_{j}$ and are uniquely defined. Note that we do not suppose that $\tau_{j} \neq \beta_{j}$.

Suppose first that $\ell>\tau_{j}$. We have defined in the proof of Theorem 22 $\mathscr{A}_{j}^{k}=\varnothing$, for all $k>\tau_{j}$. Clearly, we can freely choose the sets $\mathscr{A}_{i}^{k}$ for all $k>\tau_{j}$ to be arbitrary subsets of $\mathscr{A}_{j}^{\tau_{j}}$ provided that this arbitrary choice is consistent with the constraints $\mathscr{A}_{j}^{k} \subseteq \mathscr{A}_{j}^{k-1}$. In this case, the representation will not be unique.

Suppose now that $\ell<\beta_{j}$. We have defined in the proof of Theorem 22 $\mathscr{A}_{j}^{k}=\mathscr{A}_{j}^{\beta_{j}}$, for all $k<\beta_{j}$. Clearly, we can freely choose the sets $\mathscr{A}_{j}^{k}$ for all $k<\beta_{j}$ to be arbitrary supersets of $\mathscr{A}_{j}^{\beta_{j}}$ provided that this arbitrary choice is consistent with the constraints $\mathscr{A}_{j}^{k} \subseteq \mathscr{A}_{j}^{k-1}$. Because $\mathscr{A}_{j}^{\beta_{j}}$ is a strict subset $X_{j}$, this shows that the representation will not be unique.

Using the above observations, it is clear that if $\tau_{j}=\beta_{j}$, the representation of $\left\langle C^{k}\right\rangle_{k \in R}$ in the noncompensatory sorting model will not be unique. Suppose henceforth that $\tau_{j} \neq \beta_{j}$ and that $\beta_{j} \leq \ell \leq \tau_{j}$. Let $\ell^{+}(j) \in R^{+}$be the smallest $k>\ell$ such that $j \in N$ is influent for $\left\langle C^{\geq k}, C^{<k}\right\rangle$ and $\ell^{-}(j) \in R^{+}$be the largest $k<\ell$ such that $j \in N$ is influent for $\left\langle C^{\geq k}, C^{<k}\right\rangle$. We know that $\mathscr{A}_{j}^{\ell^{+}(j)}$ and $\mathscr{A}_{j}^{\ell^{-}(j)}$ are uniquely defined and that $\mathscr{A}_{j}^{\ell^{+}(j)} \subseteq \mathscr{A}_{j}^{\ell^{-}(j)}$. As soon as $\mathscr{A}_{j}^{\ell^{+}(j)} \subsetneq \mathscr{A}_{j}^{\ell^{-}(j)}$, the representation will not be unique. Indeed, for all $k$ such that $\ell^{-}(j)<k<\ell^{+}(j)$, we have defined in the proof of Theorem 22, $\mathscr{A}_{j}^{k}=\mathscr{A}_{j}^{\ell^{+}(j)}$. Clearly, we might as well have taken all sets $\mathscr{A}_{j}^{k}$ to be such that $\mathscr{A}_{j}^{\ell^{+}(j)} \subseteq \mathscr{A}_{j}^{k} \subseteq \mathscr{A}_{j}^{\ell^{-}(j)}$, provided that this arbitrary choice is consistent with the constraints $\mathscr{A}_{j}^{k} \subseteq \mathscr{A}_{j}^{k-1}$. On the contrary, if $\mathscr{A}_{j}^{\ell^{+}(j)}=\mathscr{A}_{j}^{\ell^{-}(j)}$, the set $\mathscr{A}_{j}^{\ell}$ will be uniquely defined even though $j \in N$ is degenerate for $\left\langle C^{\geq \ell}, C^{<\ell}\right\rangle$.

We give below an example showing that there are instances of partitions in which some attributes are degenerate for some of the twofold partitions $\left\langle C^{\geq k}, C^{<k}\right\rangle$, while the representation of $\left\langle C^{k}\right\rangle_{k \in R}$ in the noncompensatory sorting model is unique. It also shows that an attribute may be influent for $\left\langle C^{\geq k+1}, C^{<k+1}\right\rangle$ and $\left\langle C^{\geq k-1}, C^{<k-1}\right\rangle$, while being degenerate for $\left\langle C^{\geq k}, C^{<k}\right\rangle$.

\section{Example 28}

Let $n=3, X=\left\{x_{1}, y_{1}\right\} \times\left\{x_{2}, y_{2}\right\} \times\left\{x_{3}, y_{3}\right\}$ and $r=4$. Let $C^{4}=$ $\left\{\left(x_{1}, x_{2}, x_{3}\right)\right\}, C^{3}=\left\{\left(y_{1}, x_{2}, x_{3}\right)\right\}, C^{2}=\left\{\left(x_{1}, x_{2}, y_{3}\right),\left(x_{1}, y_{2}, x_{3}\right)\right\}$ and $C^{1}=$ 
$\left\{\left(x_{1}, y_{2}, y_{3}\right),\left(y_{1}, x_{2}, y_{3}\right),\left(y_{1}, y_{2}, x_{3}\right),\left(y_{1}, y_{2}, y_{3}\right)\right\}$

All attributes are influent for the twofold partition $\left\langle C^{\geq 4}, C^{<4}\right\rangle$. It has the unique representation $\mathscr{A}_{1}^{4}=\left\{x_{1}\right\}, \mathscr{A}_{2}^{4}=\left\{x_{2}\right\}, \mathscr{A}_{3}^{4}=\left\{x_{3}\right\}$ and $\mathscr{F}^{4}=\{N\}$.

All attributes are influent for $\left\langle C^{\geq 2}, C^{<2}\right\rangle$. It has the unique representation $\mathscr{A}_{1}^{2}=\left\{x_{1}\right\}, \mathscr{A}_{2}^{2}=\left\{x_{2}\right\}, \mathscr{A}_{3}^{2}=\left\{x_{3}\right\}$ and $\mathscr{F}^{2}=\{\{1,2\},\{1,3\},\{2,3\}$, $\{1,2,3\}\}$.

Attributes 2 and 3 are influent for $\left\langle C^{\geq 3}, C^{<3}\right\rangle$ while attribute 1 is degenerate. In order to satisfy the constraints of the noncompensatory sorting model, the representation of $\left\langle C^{\geq 3}, C^{<3}\right\rangle$ must be chosen so that $\mathscr{A}_{1}^{3}=\left\{x_{1}\right\}$, $\mathscr{A}_{2}^{3}=\left\{x_{2}\right\}, \mathscr{A}_{3}^{3}=\left\{x_{3}\right\}$ and $\mathscr{F}^{2}=\{\{2,3\},\{1,2,3\}\}$. Hence, $\left\langle C^{k}\right\rangle_{k \in R}$ has a unique representation.

The uniqueness of the representation in the noncompensatory sorting model is therefore stronger than what it is in the particular case of twofold partitions: it may happen that an attribute is degenerate for a twofold partition $\left\langle C^{\geq k}, C^{<k}\right\rangle$ while the representation of $\left\langle C^{k}\right\rangle_{k \in R}$ is unique. It is nevertheless apparent that this is rather an exceptional situation so that in general, the representation of a partition in the noncompensatory sorting model will not be unique when an attribute is degenerate for one of the twofold partitions $\left\langle C^{\geq k}, C^{<k}\right\rangle$. As detailed in Bouyssou and Marchant (2005), this shows that methods designed to infer the parameters of an ELECTRE TRI model on the basis of assignment examples (e.g., Dias et al., 2002; Mousseau et al., 2001a; Mousseau and Słowiński, 1998; Ngo The and Mousseau, 2002), should be prepared to deal with such situations.

\section{Remark 29}

Consider a partition $\left\langle C^{k}\right\rangle_{k \in R}$ that has a unique representation in the noncompensatory sorting model. Take $S_{i}=\succsim_{i}^{R}$ and $V_{i}=\varnothing$, for all $i \in N$. Since $\left\langle C^{k}\right\rangle_{k \in R}$ is $R$-linear, the relations $S_{i}$ are weak orders and, hence, semiorders. Suppose, for simplicity, that the sets $\mathscr{A}_{i}^{r} \subseteq \mathscr{A}_{i}^{r-1} \subseteq \cdots \subseteq \mathscr{A}_{i}^{k} \subseteq \cdots \subseteq \mathscr{A}_{i}^{2}$ are all distinct and nonempty. For all $i \in N$, take $p_{i}^{k}$ to be any element in $\mathscr{A}_{i}^{k} \backslash \mathscr{A}_{i}^{k+1}$ (with the convention that $\mathscr{A}_{i}^{r+1}=\varnothing$ ). It is easy to see that with such choices, we have We have $x_{i} S_{i} p_{i}$ iff $x_{i} \in \mathscr{A}_{i}$, so that

$$
x \in C^{\geq k} \Leftrightarrow\left\{i \in N: x_{i} S_{i} p_{i}^{k}\right\} \in \mathscr{F}^{k} .
$$

Observe that if the sets $\mathscr{F}^{k}$ have a joint additive representation à la ELECTRE, i.e., that, for all $i \in N$, there are nonnegative weights $w_{i}$ and a real number $\lambda^{k}$ between $1 / 2$ and 1 such that, for all $I \subseteq 2^{N}$,

$$
I \in \mathscr{F}^{k} \Leftrightarrow \sum_{i \in I} w_{i} \geq \lambda^{k}
$$


the above construction is exactly equivalent to the concordance part of ELECTRE TRI with nested relations.

We leave to the reader the, easy, task of extending this construction to cover the case in which some of the sets $\mathscr{A}_{i}^{k}$ are empty or when $\mathscr{A}_{i}^{k}=\mathscr{A}_{i}^{k+1}$.

Neglecting additivity issues, this shows that a partition that can be obtained with ELECTRE TRI (with $V_{i}=\varnothing$, for all $i \in N$ ) using profiles that are outside the set $X$ can always be obtained with ELECTRE TRI (still with $V_{i}=\varnothing$, for all $\left.i \in N\right)$ using profiles that belong to $X$.

\subsection{Extensions}

This section is devoted to the study of two particular cases of the noncompensatory sorting model. It may be skipped without loss of continuity.

\subsubsection{The case $\mathscr{A}_{i}^{k}=\mathscr{A}_{i}^{\ell}$}

We analyze here what must be added to the conditions in Theorem 22, in order to ensure that $\left\langle C^{k}\right\rangle_{k \in R}$ has a representation in the noncompensatory sorting model in which $\mathscr{A}_{i}^{k}=\mathscr{A}_{i}^{\ell}$, for all $k, \ell \in R^{+}$. With such a model, going from $C^{\geq k}$ to $C^{\geq k-1}$ only involves a change from $\mathscr{F}^{k}$ to $\mathscr{F}^{k-1}$, i.e., a change in the "strength" of the coalition of attributes needed to ensure that an alternative is judged satisfactory.

Suppose that $\left\langle C^{k}\right\rangle_{k \in R}$ has a representation in the noncompensatory sorting model such that $\mathscr{A}_{i}^{k}=\mathscr{A}_{i}^{\ell}$, for all $k, \ell \in R^{+}$. Let $k \in R^{+}$and suppose that $\left(x_{i}, a_{-i}\right) \in C^{\geq k}$ and $\left(y_{i}, a_{-i}\right) \in C^{<k}$. This implies that $x_{i} \in \mathscr{A}_{i}^{k}$ and $y_{i} \notin \mathscr{A}_{i}^{k}$. Let $\ell \in R^{+}$such that $\ell<k$. Since, by hypothesis, $\mathscr{A}_{i}^{k}=\mathscr{A}_{i}^{\ell}$, this implies that $y_{i} \notin \mathscr{A}_{i}^{\ell}$. Therefore, if $\left(y_{i}, b_{-i}\right) \in C^{\geq \ell}$, it must be true that $\left(z_{i}, b_{-i}\right) \in C^{\geq \ell}$, for all $z_{i} \in X_{i}$. This shows that $\left\langle C^{k}\right\rangle_{k \in R}$ satisfies the following condition:

$$
\left.\begin{array}{c}
\left(x_{i}, a_{-i}\right) \in C^{\geq k} \\
\text { and } \\
\left(y_{i}, b_{-i}\right) \in C^{\geq \ell}
\end{array}\right\} \Rightarrow\left\{\begin{array}{c}
\left(y_{i}, a_{-i}\right) \in C^{\geq k} \\
\text { or } \\
\left(z_{i}, b_{-i}\right) \in C^{\geq \ell}
\end{array}\right.
$$

for all $k, \ell \in R^{+}$such that $\ell<k$, all $x_{i}, y_{i}, z_{i} \in X_{i}$, and all $a_{-i}, b_{-i} \in X_{-i}$. We say that $\left\langle C^{k}\right\rangle_{k \in R}$ satisfies condition $E q$ if it satisfies condition $E q_{i}$ for all $i \in N$.

The interpretation of condition $E q_{i}$ is easy considering its impact on the relations $\succsim_{i}^{k}$. We have:

\section{Lemma 30}

A partition $\left\langle C^{k}\right\rangle_{k \in R}$ satisfies condition $E q_{i}$ iff for all $k, \ell \in R$ with $\ell<k$ and all $x_{i}, y_{i}, z_{i} \in X_{i}, \operatorname{Not}\left[y_{i} \succsim_{i}^{k} x_{i}\right] \Rightarrow z_{i} \succsim_{i}^{\ell} y_{i}$. 


\section{PROOF}

Suppose that, for some $k, \ell \in R$ with $\ell<k$ and some $x_{i}, y_{i}, z_{i} \in X_{i}$, we have $\operatorname{Not}\left[y_{i} \succsim_{i}^{k} x_{i}\right]$ and $\operatorname{Not}\left[z_{i} \succsim_{i}^{\ell} y_{i}\right]$. By definition this is equivalent to saying that, for some $a_{-i}, b_{-i} \in X_{-i},\left(x_{i}, a_{-i}\right) \in C^{\geq k},\left(y_{i}, a_{-i}\right) \notin C^{\geq k},\left(y_{i}, b_{-i}\right) \in C^{\geq \ell}$ and $\left(z_{i}, b_{-i}\right) \notin C^{\geq \ell}$. This is equivalent to saying that $E q_{i}$ is violated.

We have:

\section{Proposition 31}

An $r$-fold partition $\left\langle C^{k}\right\rangle_{k \in R}$ of $X$ has a representation in the noncompensatory sorting model with $\mathscr{A}_{i}^{k}=\mathscr{A}_{i}^{\ell}$, for all $k, \ell \in R^{+}$iff it is $R$-linear, $R$-2-graded and satisfies Eq.

\section{Proof}

The necessity of $R$-linearity and $R$-2-gradedness follows from Theorem 22 . The necessity of $E q$ was shown above.

Sufficiency. Since $\left\langle C^{k}\right\rangle_{k \in R}$ is $R$-linear and $R$-2-graded, it has a representation in the noncompensatory sorting model. Let $\left\langle\mathscr{F}^{k},\left\langle\mathscr{A}_{i}^{k}\right\rangle_{i \in N}\right\rangle$ be the representation built in Theorem 22. Suppose that, for some $\ell \in R^{+}$with $\ell<r$, we have $x_{i} \in \mathscr{A}_{i}^{\ell}$. If attribute $i \in N$ is degenerate for $\left\langle C^{\geq \ell}, C^{<\ell}\right\rangle$, we have $\mathscr{A}_{i}^{\ell}=\mathscr{A}_{i}^{\ell+1}$ and there is nothing to prove. Hence, suppose that attribute $i \in N$ is influent for $\left\langle C^{\geq \ell}, C^{<\ell}\right\rangle$, so that $\left(x_{i}, c_{-i}\right) \in C^{\geq \ell}$ and $\left(z_{i}, c_{-i}\right) \in C^{<\ell}$, for some $z_{i} \in X_{i}$ and some $c_{-i} \in X_{-i}$.

Suppose first that attribute $i \in N$ is degenerate for all $k>\ell$. We have $\mathscr{A}_{i}^{k}=\varnothing$, for all $k>\ell$. However, as shown in Section 5.7, this representation is not unique and it is always possible to take all sets $\mathscr{A}_{i}^{k}$ to be equal to $\mathscr{A}_{i}^{\ell}$, so that we will have $\mathscr{A}_{i}^{\ell}=\mathscr{A}_{i}^{\ell+1}$.

Suppose now that attribute $i \in N$ is influent for $\left\langle C^{\geq k}, C^{<k}\right\rangle$, for some $k>$ $\ell$. Let $k^{*}$ be the smallest $k>\ell$ such that $i \in N$ is influent for $\left\langle C^{\geq k^{*}}, C^{<k^{*}}\right\rangle$, so that $\mathscr{A}_{i}^{k^{*}}=\mathscr{A}_{i}^{\ell+1}$. Suppose that $x_{i} \notin \mathscr{A}_{i}^{k^{*}}$. This implies $\left(y_{i}, a_{-i}\right) \in C^{\geq k^{*}}$ and $\left(x_{i}, a_{-i}\right) \in C^{<k^{*}}$, for some $y_{i} \in X_{i}$ and some $a_{-i} \in X_{-i}$. Using $E q_{i}$, $k^{*}>\ell,\left(y_{i}, a_{-i}\right) \in C^{\geq k^{*}}$ and $\left(x_{i}, c_{-i}\right) \in C^{\geq \ell}$ imply either $\left(x_{i}, a_{-i}\right) \in C^{\geq k^{*}}$ or $\left(z_{i}, c_{-i}\right) \in C^{\geq \ell}$, a contradiction.

Hence, we have shown that there is a representation such that $\mathscr{A}_{i}^{\ell}=$ $\mathscr{A}_{i}^{\ell+1}$, for all $\ell<r$. This completes the proof.

Let us observe that none of the conditions used in Proposition 31 is redundant. Using an ELECTRE TRI model, it is easy to build partitions that are $R$-linear, $R$-2-graded and satisfy condition $E q_{i}$ on all but one attribute. We give below the other two examples.

\section{Example 32}

Let $n=2, X=\left\{x_{1}, y_{1}, z_{1}\right\} \times\left\{x_{2}, y_{2}\right\}$ and $r=3$. Let $C^{3}=\left\{\left(x_{1}, x_{2}\right),\left(y_{1}, x_{2}\right)\right.$, $\left.\left(y_{1}, y_{2}\right)\right\}, C^{2}=\left\{\left(z_{1}, x_{2}\right)\right\}$ and $C^{1}=\left\{\left(x_{1}, y_{2}\right),\left(z_{1}, y_{2}\right)\right\}$. We have $y_{1} \succ_{1}^{R} x_{1} \succ_{1}^{R}$ 
$z_{1}$ and $x_{2} \succ_{2}^{R} y_{2}$, so that this partition is $R$-linear. Condition 2 -graded ${ }_{2}^{R}$ is trivially satisfied. Condition 2 -graded ${ }_{1}^{R}$ is violated because $\left(x_{1}, x_{2}\right) \in C^{3}$, $\left(y_{1}, x_{2}\right) \in C^{3}$ and $\left(y_{1}, y_{2}\right) \in C^{3}$ but neither $\left(x_{1}, y_{2}\right) \in C^{\geq 3}$ not $\left(z_{1}, x_{2}\right) \in C^{\geq 3}$.

We have $y_{1} \succ_{1}^{3} x_{1} \succ_{1}^{3} z_{1}$ and $y_{1} \succ_{1}^{2}\left[x_{1} \sim_{1}^{2} z_{1}\right]$. Similarly, we have $x_{2} \succ_{2}^{3} y_{2}$ and $x_{2} \succ_{2}^{2} y_{2}$. Using Lemma 30, it is easy to see that condition Eq holds. $\diamond$

\section{Example 33}

Let $n=3$ and $X=\left\{x_{1}, y_{1}\right\} \times\left\{x_{2}, y_{2}\right\} \times\left\{x_{3}, y_{3}\right\}$ and $r=3$. Let $C^{3}=$ $\left\{\left(x_{1}, x_{2}, x_{3}\right),\left(y_{1}, x_{2}, x_{3}\right)\right\}, C^{2}=\left\{\left(x_{1}, x_{2}, y_{3}\right),\left(y_{1}, y_{2}, x_{3}\right)\right\}$ and $C^{1}=\left\{\left(x_{1}, y_{2}, y_{3}\right)\right.$, $\left.\left(y_{1}, y_{2}, y_{3}\right),\left(x_{1}, y_{2}, x_{3}\right),\left(y_{1}, x_{2}, y_{3}\right)\right\}$. Since all sets $X_{i}$ have two only elements, this partition is trivially $R$-2-graded. Condition linear $_{2}$ and linear 3 hold with $x_{2} \succ_{2}^{R} y_{2}$ and $x_{3} \succ_{3}^{R} y_{3}$. Condition linear 1 is violated since $\left(x_{1}, x_{2}, y_{3}\right) \in C^{2}$, $\left(y_{1}, y_{2}, x_{3}\right) \in C^{2}$ but neither $\left(y_{1}, x_{2}, y_{3}\right) \in C^{\geq 2}$ nor $\left(x_{1}, y_{2}, x_{3}\right) \in C^{\geq 2}$.

We have $x_{1} \sim_{1}^{3} y_{1}, \operatorname{Not}\left[x_{1} \sim_{1}^{2} y_{1}\right]$ and $\operatorname{Not}\left[y_{1} \sim_{1}^{2} x_{1}\right]$. We also have $x_{i} \succ_{i}^{3} y_{i}$ and $x_{i} \succ_{i}^{2} y_{i}$, for $i \in\{2,3\}$. Using Lemma 30, it is easy to see that condition Eq holds.

\subsubsection{The case $\mathscr{F}^{k}=\mathscr{F}^{\ell}$}

We analyze here what must be added to the conditions in Theorem 22 in order to ensure that $\left\langle C^{k}\right\rangle_{k \in R}$ has a representation in the noncompensatory sorting model in which $\mathscr{F}^{k}=\mathscr{F}^{\ell}$, for all $k, \ell \in R^{+}$. In this case, going from $C^{\geq k}$ to $C^{\geq k-1}$ only involves a change in the definition of the sets $\mathscr{A}_{i}^{k}$. This restriction brings the noncompensatory sorting model closer to the original version of ELECTRE TRI. This case is more difficult to analyze than the preceding one since, in our proofs, the construction of the sets $\mathscr{F}^{k}$ is left implicit. This is a clear drawback of our proof technique.

Take any $x \in C^{k}$ and let $J(x)$ be the subset of attributes $i \in N$ such that:

$$
\begin{aligned}
& \left(z_{i}, x_{-i}\right) \in C^{<k}, \\
& \left(y_{i}, y_{-i}\right) \in C^{\geq k+1} \text { and } \\
& \left(x_{i}, y_{-i}\right) \in C^{<k+1},
\end{aligned}
$$

for some $y \in X$ and some $z_{i} \in X_{i}$. Hence, $J(x)$ is the subset of attributes $i \in N$ such that $x_{i} \in \mathscr{A}_{i}^{k}$ (because $\left(x_{i}, x_{-i}\right) \in C^{k}$ and $\left(z_{i}, x_{-i}\right) \in C^{<k}$ ) while $x_{i} \notin \mathscr{A}_{i}^{k+1}$ (because $\left(y_{i}, y_{-i}\right) \in C^{\geq k+1}$ and $\left.\left(x_{i}, y_{-i}\right) \in C^{<k+1}\right)$.

Let $x \in C^{k}$. For all $j \in J(x)$, let $y^{j} \in X$ be any alternative such that (21b) is satisfied. Consider an alternative $w \in X$ such that $w_{j}=x_{j}$ if $j \notin J(x)$ and $w_{j}=y_{j}^{j}$ if $j \in J(x)$. The alternative $w$ is identical to $x$ on all attributes such that $x_{i} \in \mathscr{A}_{i}^{k+1}$. The same is true on all attributes such 
that $x_{i} \notin \mathscr{A}_{i}^{k}$. On all attributes such that $x_{i} \in \mathscr{A}_{i}^{k}$ and $x_{i} \notin \mathscr{A}_{i}^{k+1}$, we have $w_{i} \in \mathscr{A}_{i}^{k+1}$. Therefore, we have $A^{k+1}(w)=A^{k}(x)$. Because $x \in C^{k}$, we know that $A^{k}(x) \subseteq I$ implies $I \in \mathscr{F}^{k}$. If it is required that $w \in C^{\geq k+1}$, all such subsets $I$ will also belong to $\mathscr{F}^{k+1}$.

We have exhibited a necessary condition for the existence of a representation in which $\mathscr{F}^{k}=\mathscr{F}^{k+1}$ (for all $x \in C^{k}$, the alternative $w \in X$ as built above must belong to $C^{\geq k+1}$ ). When added to $R$-linearity and $R$-2gradedness, it is not difficult to show that this condition is also sufficient to ensure the existence of such a representation. Since this new condition is quite cumbersome and the proof is not very instructive, we do not formalize this point further here. We are not presently aware of a more satisfactory characterization of this particular case of the noncompensatory sorting model, in spite of its intuitive appeal.

\section{The noncompensatory sorting model with veto}

This section extends the results of the preceding section to allow for possible veto effects, as the ELECTRE TRI method.

\subsection{Definitions}

We consider a model generalizing the noncompensatory sorting model in order to allow for possible veto effects. We say that $\left\langle C^{k}\right\rangle_{k \in R}$ has a representation in the noncompensatory sorting model with veto if:

- for all $i \in N$ and all $k \in R^{+}$there are disjoint sets $\mathscr{A}_{i}^{k}, \mathscr{V}_{i}^{k} \subseteq X_{i}$ such that:

(i) for all $i \in N, \mathscr{A}_{i}^{r} \subseteq \mathscr{A}_{i}^{r-1} \subseteq \cdots \subseteq \mathscr{A}_{i}^{2}$,

(ii) for all $i \in N, \mathscr{V}_{i}^{r} \supseteq \mathscr{V}_{i}^{r-1} \supseteq \cdots \supseteq \mathscr{V}_{i}^{2}$,

(iii) for all $k, \ell \in R^{+}$such that $k<\ell$, if $x_{i} \in \mathscr{A}_{i}^{k}, y_{i} \in \mathscr{U}_{i}^{k}$ and $x_{i} \in \mathscr{V}_{i}^{\ell}$ then $y_{i} \in \mathscr{V}_{i}^{\ell}$, where, in Section $6, \mathscr{U}_{i}^{k}=X_{i} \backslash\left[\mathscr{A}_{i}^{k} \cup \mathscr{V}_{i}^{k}\right]$,

- there are subsets $\mathscr{F}^{r}, \mathscr{F}^{r-1}, \ldots, \mathscr{F}^{2}$ of $2^{N}$ that are such that, for all $k \in R^{+}$and all $I, J \in 2^{N}$,

$$
\left[I \in \mathscr{F}^{k} \text { and } I \subseteq J\right] \Rightarrow J \in \mathscr{F}^{k},
$$

and are nested, i.e., such that,

$$
\mathscr{F}^{r} \subseteq \mathscr{F}^{r-1} \subseteq \cdots \subseteq \mathscr{F}^{2},
$$


such that:

$$
x \in C^{\geq k} \Leftrightarrow\left[\left\{i \in N: x_{i} \in \mathscr{A}_{i}^{k}\right\} \in \mathscr{F}^{k} \text { and }\left\{i \in N: x_{i} \in \mathscr{V}_{i}^{k}\right\}=\varnothing\right],
$$

for all $x \in X$ and all $k \in R^{+}$. As before we note $A^{k}(x)$ and $V^{k}(x)$ instead of $\left\{i \in N: x_{i} \in \mathscr{A}_{i}^{k}\right\}$ and $\left\{i \in N: x_{i} \in \mathscr{V}_{i}^{k}\right\}$ when there is no ambiguity on the underlying sets $\mathscr{A}_{i}^{k}$ and $\mathscr{V}_{i}^{k}$.

The interpretation of the noncompensatory sorting model with veto is similar to that of the noncompensatory sorting model, the latter being a particular case of the former. The only difference is that, for each $k \in R^{+}$, there is a set $\mathscr{V}_{i}^{k}$ that is repulsive for $C^{\geq k}$. Since the categories are ordered, the requirement that a level that is repulsive for a given category should be repulsive for all higher categories is not surprising. This explains the introduction of the additional constraints $\mathscr{V}_{i}^{k} \supseteq \mathscr{V}_{i}^{k-1}$. Condition (iii) is a consistency requirement on $\mathscr{A}_{i}^{k}, \mathscr{U}_{i}^{k}$ and $\mathscr{V}_{i}^{\ell}$ that can be interpreted as follows. If $x_{i} \in \mathscr{A}_{i}^{k}, y_{i} \in \mathscr{U}_{i}^{k}$, we have an indication that $x_{i}$ is superior to $y_{i}$. Supposing now that, for some $\ell>k, x_{i} \in \mathscr{V}_{i}^{\ell}$ and $y_{i} \notin \mathscr{V}_{i}^{\ell}$, would give the inconsistent indication that $y_{i}$ is superior to $x_{i}$.

The pessimistic version of ELECTRE TRI with nested relations (and, hence, the pessimistic version of ELECTRE TRI) is a particular case of the noncompensatory sorting model with veto. Indeed, remember from Section 4 that in the pessimistic version of ELECTRE TRI with nested relations we have, for all $x \in X$, and all $k \in R^{+}$,

$$
x \in C^{\geq k} \Leftrightarrow\left[\sum_{i \in S\left(x, p^{k}\right)} w_{i} \geq \lambda^{k} \text { and }\left[\operatorname{Not}\left[p_{i}^{k} V_{i} x_{i}\right], \text { for all } i \in N\right]\right] .
$$

Define $\mathscr{A}_{i}^{k}=\left\{i \in N: x_{i} S_{i} p^{k}\right\}, \mathscr{V}_{i}^{k}=\left\{i \in N: p^{k} V_{i} x_{i}\right\}$ and let $I \in \mathscr{F}^{k}$ whenever $\sum_{i \in I} w_{i} \geq \lambda^{k}$.

By construction, $x_{i} \in \mathscr{A}_{i}^{k}$ implies $x_{i} S_{i} p_{i}^{k}$. Since $\left.p_{i}^{k}\right) T_{i} p_{i}^{k-1}$, we obtain $x_{i} S_{i} p_{i}^{k-1}$, so that $x_{i} \in \mathscr{A}_{i}^{k-1}$. The proof that $\mathscr{V}_{i}^{k-1} \subseteq \mathscr{V}_{i}^{k}$ is similar. The sets $\mathscr{A}_{i}^{k}$ and $\mathscr{V}_{i}^{k}$ are disjoint. Suppose now that $k<\ell, x_{i} \in \mathscr{A}_{i}^{k}$, $y_{i} \in \mathscr{U}_{i}^{k}$ and $x_{i} \in \mathscr{V}_{i}^{\ell}$. This implies $x_{i} S_{i} p_{i}^{k}, p_{i}^{k} P_{i} y_{i}$ and $p_{i}^{\ell} V_{i} x_{i}$. The first two equations imply $x_{i} T_{i} y_{i}$. The third equation therefore implies that $p_{i}^{k} T_{i} y_{i}$, so that $y_{i} \in \mathscr{V}_{i}^{\ell}$. Because $\lambda^{k} \geq \lambda^{k-1}$, we have $\mathscr{F}^{k} \subseteq \mathscr{F}^{k-1}$. Hence, $\left\langle\mathscr{F}^{k},\left\langle\mathscr{A}_{i}^{k}, \mathscr{V}_{i}^{k}\right\rangle_{i \in N}\right\rangle$ is a representation of $\left\langle C^{k}\right\rangle_{k \in R}$ in the noncompensatory sorting model with veto.

\section{Remark 34}

In the noncompensatory sorting model with veto, as soon as $x_{i} \in \mathscr{V}_{i}^{k}$, it is impossible to have $\left(x_{i}, a_{-i}\right) \in C^{\geq k}$, for all $a_{-i} \in X_{-i}$. This idea of "discordance" is simple but therefore rather radical: levels that, on their own, 
are not repulsive cannot "interact" so that their combination would be repulsive. Extensions of the noncompensatory sorting model with veto that would tolerate some of these interactions could well lead to interesting models on the theoretical side, while remaining sufficiently simple so as to be useful in practice. Such models could be the subject of future research.

\subsection{Axioms}

The noncompensatory sorting model with veto shares with the noncompensatory sorting model the fact that it implies $R$-linearity.

\section{Lemma 35}

If a partition $\left\langle C^{k}\right\rangle_{k \in R}$ has a representation in the noncompensatory sorting model with veto then it is $R$-linear.

PROOF

Suppose that linear $_{i}^{R}$ is violated so that, for some $k, \ell \in R^{+}$, some $x_{i}, y_{i} \in X_{i}$, and some $a_{-i}, b_{-i} \in X_{-i},\left(x_{i}, a_{-i}\right) \in C^{k},\left(y_{i}, b_{-i}\right) \in C^{\ell},\left(y_{i}, a_{-i}\right) \in C^{<k}$ and $\left(x_{i}, b_{-i}\right) \in C^{<\ell}$. Suppose w.l.o.g. that $k \leq \ell$. Because $\left(x_{i}, a_{-i}\right) \in C^{k}$ and $\left(y_{i}, a_{-i}\right) \in C^{<k}$, we have either $y_{i} \in \mathscr{U}_{i}^{k}$ or $y_{i} \in \mathscr{V}_{i}^{k}$. Because $k \leq \ell$, we know that $\mathscr{V}_{i}^{\ell} \supseteq \mathscr{V}_{i}^{k}$. It is therefore impossible that $y_{i} \in \mathscr{V}_{i}^{k}$ since this would imply $y_{i} \in \mathscr{V}_{i}^{\ell}$, contradicting $\left(y_{i}, b_{-i}\right) \in C^{\ell}$. Hence, we must have $y_{i} \in \mathscr{U}_{i}^{k}$, so that $x_{i} \in \mathscr{A}_{i}^{k}$. Because $\mathscr{A}_{i}^{\ell} \subseteq \mathscr{A}_{i}^{k}$, we know that $y_{i} \in \mathscr{U}_{i}^{\ell}$.

Because $\left(y_{i}, b_{-i}\right) \in C^{\ell},\left(x_{i}, b_{-i}\right) \in C^{<\ell}$ and $y_{i} \in \mathscr{U}_{i}^{\ell}$, we must have $x_{i} \in \mathscr{V}_{i}^{\ell}$. Since we have $x_{i} \in \mathscr{A}_{i}^{k}, x_{i} \in \mathscr{V}_{i}^{\ell}$ and $y_{i} \in \mathscr{U}_{i}^{k}$, the definition of the generalized ordinal sorting model with veto implies that $y_{i} \in \mathscr{V}_{i}^{\ell}$, contradicting the fact that $\left(y_{i}, b_{-i}\right) \in C^{\ell}$.

Similarly to what was done before, we wish to add to $R$-linearity conditions that would precipitate the noncompensatory sorting model with veto. We say that $\left\langle C^{k}\right\rangle_{k \in R}$ is $R$-3v-graded on attribute $i \in N\left(\right.$ condition $3 v$-graded ${ }_{i}^{R}$ ) if:

$$
\left.\begin{array}{l}
\left(x_{i}, a_{-i}\right) \in C^{\geq k} \\
\text { and } \\
\left(y_{i}, a_{-i}\right) \in C^{\geq k} \\
\text { and } \\
\left(y_{i}, b_{-i}\right) \in C^{\geq \ell} \\
\text { and } \\
\left(z_{i}, c_{-i}\right) \in C^{\geq k}
\end{array}\right\} \Rightarrow\left\{\begin{array}{c}
\left(x_{i}, b_{-i}\right) \in C^{\geq \ell} \\
\text { or } \\
\left(z_{i}, a_{-i}\right) \in C^{\geq k}
\end{array} \quad\left(3 \text { voraded }_{i}^{R}\right)\right.
$$

for all $k, \ell \in R^{+}$such that $\ell \leq k$, all $x_{i}, y_{i}, z_{i} \in X_{i}$, and all $a_{-i}, b_{-i} \in X_{-i}$. We say that $\left\langle C^{k}\right\rangle_{k \in R}$ is $R$-3v-graded if condition $3 \mathrm{v}$-graded ${ }_{i}^{R}$ holds for all $i \in N$. This condition generalizes the condition $3 \mathrm{v}$-graded $i$ introduced in Bouyssou 
and Marchant (2005), this condition being in turn, inspired by Greco et al. (2001a) who study veto effects in the context of binary relations.

Note that condition $3 \mathrm{v}$-graded ${ }_{i}^{R}$ is the weakening of 2 -graded ${ }_{i}^{R}$ obtained by adding to it the premise $\left(z_{i}, c_{-i}\right) \in C^{\geq k}$. The intuition behind this weakening is that the noncompensatory sorting model with veto requires condition 2 -graded ${ }_{i}^{R}$ to hold for elements that are not repulsive. Adding the premise $\left(z_{i}, c_{-i}\right) \in C^{\geq k}$ ensures that $z_{i} \notin \mathscr{V}_{i}^{k}$.

\section{Lemma 36}

If a partition $\left\langle C^{k}\right\rangle_{k \in R}$ has a representation in the noncompensatory sorting model with veto then it is $R$-3v-graded.

\section{ProOF}

Suppose that, for some $k, \ell \in R^{+}$such that $\ell \leq k$, some $x_{i}, y_{i}, z_{i} \in X_{i}$, and some $a_{-i}, b_{-i}, c_{-i} \in X_{-i},\left(x_{i}, a_{-i}\right) \in C^{\geq k},\left(y_{i}, b_{-i}\right) \in C^{\geq \ell},\left(z_{i}, c_{-i}\right) \in C^{\geq k}$ and $\left(z_{i}, a_{-i}\right) \in C^{<k}$. Because $\left(z_{i}, c_{-i}\right) \in C^{\geq k}$, it is impossible that $z_{i} \in \mathscr{V}_{i}^{k}$. Hence, $\left(x_{i}, a_{-i}\right) \in C^{\geq k}$ and $\left(z_{i}, a_{-i}\right) \in C^{<k}$ imply $x_{i} \in \mathscr{A}_{i}^{k}$. Because $\ell \leq k$, we know that $\mathscr{A}_{i}^{k} \subseteq \mathscr{A}_{i}^{\ell}$. Hence, we have $x_{i} \in \mathscr{A}_{i}^{\ell}$. Since $\left(y_{i}, b_{-i}\right) \in C^{\geq \ell}$, the fact that $\mathscr{F}^{\ell}$ satisfies $(22)$ implies $\left(x_{i}, b_{-i}\right) \in C^{\geq \ell}$.

The following lemma summarizes the consequences of conditions $\operatorname{linear}_{i}^{R}$ and $3 v$-graded ${ }_{i}^{R}$ using the relations $\succsim_{i}^{k}$.

\section{Lemma 37}

Conditions linear ${ }_{i}^{R}$ and $3 \mathrm{v}$-graded ${ }_{i}^{R}$ hold iff the following four conditions hold:

(a) $\succsim_{i}^{k}$ is a weak order having at most three distinct equivalence classes.

(b) If $\succsim_{i}^{k}$ has three distinct equivalence classes and $x_{i}$ is in the last class then, for all $a_{-i} \in X_{-i},\left(x_{i}, a_{-i}\right) \notin C^{\geq k}$,

(c) $\left[x_{i} \succ_{i}^{k} y_{i}\right] \Rightarrow\left[x_{i} \succsim_{i}^{\ell} y_{i}\right.$, for all $\left.\ell \in R^{+}\right]$,

(d) $\left[x_{i} \sim_{i}^{k} z_{i}\right.$ and $x_{i} \succ_{i}^{k} y_{i}$ and $\left(y_{i}, a_{-i}\right) \in C^{\geq k}$, for some $\left.a_{-i} \in X_{-i}\right] \Rightarrow$ $\left[x_{i} \sim_{i}^{\ell} z_{i}\right.$, for all $\left.\ell<k\right]$.

for all $k \in R^{+}$and all $x_{i}, y_{i}, z_{i} \in X_{i}$.

\section{Proof}

Part $[\Leftarrow]$. Suppose that linear $_{i}^{R}$ is violated so that, for some $x_{i}, y_{i} \in X_{i}$ and some $a_{-i}, b_{-i} \in X_{-i},\left(x_{i}, a_{-i}\right) \in C^{k},\left(y_{i}, b_{-i}\right) \in C^{\ell},\left(y_{i}, a_{-i}\right) \in C^{<k}$ and $\left(x_{i}, b_{-i}\right) \in C^{<\ell}$. Using (a), we know that for all $k \in R^{+}, \succsim_{i}^{k}$ is a weak order. The above relations therefore imply that $x_{i} \succ_{i}^{k} y_{i}$ and $y_{i} \succ_{i}^{\ell} x_{i}$, contradicting (c). 
Suppose that $3 \mathrm{~V}$-graded ${ }_{i}^{R}$ is violated so that, for some $\ell \leq k,\left(x_{i}, a_{-i}\right) \in$ $C^{\geq k},\left(y_{i}, a_{-i}\right) \in C^{\geq k},\left(y_{i}, b_{-i}\right) \in C^{\geq \ell},\left(z_{i}, c_{-i}\right) \in C^{\geq k},\left(x_{i}, b_{-i}\right) \in C^{<\ell}$ and $\left(z_{i}, a_{-i}\right) \in C^{<k}$. Using (a), this implies $y_{i} \succ_{i}^{\ell} x_{i}, x_{i} \succ_{i}^{k} z_{i}$ and $y_{i} \succ_{i}^{k} z_{i}$.

Suppose first that $k=\ell$. We obtain $y_{i} \succ_{i}^{k} x_{i}$ and $x_{i} \succ_{i}^{k} z_{i}$. Since $\left(z_{i}, c_{-i}\right) \in C^{\geq k}$, this contradicts (b).

Suppose henceforth that $k>\ell$. Using (c), $y_{i} \succ_{i}^{\ell} x_{i}$ implies $y_{i} \succsim_{i}^{k} x_{i}$. Since $x_{i} \succ_{i}^{k} z_{i}$ and $\left(z_{i}, c_{-i}\right) \in C^{\geq k}$, using (b) implies that we cannot have $y_{i} \succ_{i}^{k} x_{i}$. Suppose therefore that $y_{i} \sim_{i}^{k} x_{i}$. Using (d), $x_{i} \sim_{i}^{k} y_{i}, x_{i} \succ_{i}^{k} z_{i}$ and $\left(z_{i}, c_{-i}\right) \in C^{\geq k}$ would imply $x_{i} \sim_{i}^{\ell} y_{i}$, a contradiction.

Part $[\Rightarrow]$. Using linear $i_{i}^{R}$, we know that $\succsim_{i}^{R}$ is complete. Since $\succsim_{i}^{R}$ refines $\succsim_{i}^{k}$, it follows that $\succsim_{i}^{k}$ is complete and, hence, a weak order. Clearly, $x_{i} \succ_{i}^{k} y_{i}$ and $y_{i} \succ_{i}^{\ell} x_{i}$ would violate linear $_{i}^{R}$. Hence (c) holds.

Suppose that, for some $k \in R^{+}, \succsim_{i}^{k}$ has at least four distinct equivalence classes so that, for some $x_{i}, y_{i}, z_{i}, w_{i} \in X_{i}$, we have $x_{i} \succ_{i}^{k} y_{i}, y_{i} \succ_{i}^{k} z_{i}$ and $z_{i} \succ_{i}^{k} w_{i}$. Using the definition of $\succsim_{i}^{k}$, we have, for some $a_{-i}, b_{-i}, c_{-i} \in X_{-i}$, $\left(x_{i}, a_{-i}\right) \in C^{k},\left(y_{i}, a_{-i}\right) \in C^{<k},\left(y_{i}, b_{-i}\right) \in C^{k},\left(z_{i}, b_{-i}\right) \in C^{<k},\left(z_{i}, c_{-i}\right) \in C^{k}$, $\left(w_{i}, c_{-i}\right) \in C^{<k}$.

Using linear ${ }_{i}^{R},\left(x_{i}, a_{-i}\right) \in C^{k},\left(y_{i}, b_{-i}\right) \in C^{k}$ and $\left(y_{i}, a_{-i}\right) \in C^{<k}$ imply $\left(x_{i}, b_{-i}\right) \in C^{\geq k}$. Using $3 v_{-g r a d e d}^{R}$ with $\ell=k,\left(y_{i}, b_{-i}\right) \in C^{k},\left(x_{i}, b_{-i}\right) \in C^{\geq k}$, $\left(x_{i}, a_{-i}\right) \in C^{k}$ and $\left(z_{i}, c_{-i}\right) \in C^{k}$ imply either $\left(y_{i}, a_{-i}\right) \in C^{\geq k}$ or $\left(z_{i}, b_{-i}\right) \in$ $C^{\geq k}$, a contradiction. Note that this also shows that $x_{i} \succ_{i}^{k} y_{i}, y_{i} \succ_{i}^{k} z_{i}$ and $\left(z_{i}, c_{-i}\right) \in C^{k}$ is contradictory. Hence, (a) and (b) hold.

Suppose now that, for some $\ell<k$ and some $x_{i}, y_{i}, z_{i} \in X_{i}, x_{i} \sim_{i}^{k} z_{i}$, $x_{i} \succ_{i}^{k} y_{i},\left(y_{i}, a_{-i}\right) \in C^{\geq k}$ and $x_{i} \succ_{i}^{\ell} z_{i}$, the case $z_{i} \succ_{i}^{\ell} x_{i}$ being similar. By definition, $x_{i} \sim_{i}^{k} z_{i}$ and $x_{i} \succ_{i}^{k} y_{i}$ imply that $\left(x_{i}, b_{-i}\right) \in C^{k},\left(z_{i}, b_{-i}\right) \in C^{k}$ and $\left(y_{i}, b_{-i}\right) \in C^{<k}$, for some $b_{-i} \in X_{-i}$. Similarly $x_{i} \succ_{i}^{\ell} z_{i}$ implies $\left(x_{i}, c_{-i}\right) \in C^{\ell}$ and $\left(z_{i}, c_{-i}\right) \in C^{<\ell}$, for some $c_{-i} \in X_{-i}$. Using $3 v$-graded ${ }_{i}^{R},\left(z_{i}, b_{-i}\right) \in C^{k}$, $\left(x_{i}, b_{-i}\right) \in C^{k},\left(x_{i}, c_{-i}\right) \in C^{\ell}$ and $\left(y_{i}, a_{-i}\right) \in C^{\geq k}$ imply $\left(z_{i}, c_{-i}\right) \in C^{\geq \ell}$ or $\left(y_{i}, b_{-i}\right) \in C^{\geq k}$, a contradiction. Hence, (d) holds, which completes the proof.

\subsection{Result}

Our main result in this paper says that $R$-linearity and $R$-3v-gradedness characterize the noncompensatory sorting model with veto.

\section{Theorem 38}

An $r$-fold partition $\left\langle C^{k}\right\rangle_{k \in R}$ has a representation in the noncompensatory sorting model with veto iff it is $R$-linear and $R$-3v-graded.

PROOF

Necessity results from Lemmas 35 and 36. 
Sufficiency. Suppose that $\left\langle C^{k}\right\rangle_{k \in R}$ is $R$-linear and $R$-3v-graded. For all $k \in R^{+}$, define $\mathscr{V}_{i}^{k}=\left\{x_{i} \in X_{i}:\left(x_{i}, a_{-i}\right) \in C^{<k}\right.$, for all $\left.a_{-i} \in X_{-i}\right\}$. By construction, the constraints $\mathscr{V}_{i}^{k} \subseteq \mathscr{V}_{i}^{\ell}$ for $\ell>k$ are always satisfied with such a definition.

Let $Y_{i}^{k}=X_{i} \backslash \mathscr{V}_{i}^{k}$ and $Y^{k}=\prod_{i \in N} Y_{i}^{k}$. We have $Y_{i}^{k} \subseteq Y_{i}^{k-1}$, for all $k \in\{r, r-1, \ldots, 3\}$. Since $\left\langle C^{k}\right\rangle_{k \in R}$ is a partition, $\left\langle C^{\geq k}, C^{<k}\right\rangle$ is a partition, for all $k \in R^{+}$. Hence the sets $Y^{k}$ are nonempty, for all $k \in R^{+}$. Let $D^{\geq k}=C^{\geq k} \cap Y^{k}$ and $D^{<k}=C^{<k} \cap Y^{k}$.

Notice that $\left\langle D^{\geq k}, D^{<k}\right\rangle$ is not necessarily a partition since it may well happen that $D^{\geq k}=Y^{k}$ so that $D^{<k}=\varnothing$. When there is at least one attribute influent for $\left\langle D^{\geq k}, D^{<k}\right\rangle$, it will be a partition. In this case, our plan is to build a representation of $\left\langle D^{\geq k}, D^{<k}\right\rangle$ in the noncompensatory sorting model using Proposition 21. We will use the non uniqueness of such a representation to deal with degenerate attributes in such a way that the constraints of the noncompensatory sorting model with veto are satisfied. Notice that, in what follows, the set $\mathscr{A}_{i}^{k}$ will always be a subset of $Y_{i}^{k}$. Hence, the sets $\mathscr{V}_{i}^{k}$ and $\mathscr{A}_{i}^{k}$ will always be disjoint.

Suppose first that, for all $k \in R^{+}$, all attributes $i \in N$ are degenerate for $\left\langle D^{\geq k}, D^{<k}\right\rangle$. By construction of the sets $Y_{i}^{k}$, we have $D^{\geq k}=Y^{k}$. In this case, define, for all $k \in R^{+}$and all $i \in N, \mathscr{A}_{i}^{k}=Y_{i}^{k}$ and $\mathscr{F}^{k}=\{N\}$. This clearly gives a representation of $\left\langle C^{k}\right\rangle_{k \in R}$ in the noncompensatory sorting model with veto.

Otherwise, let $k \in R^{+}$be such that $\left\langle D^{\geq k}, D^{<k}\right\rangle$ is a partition. It is clear that this partition satisfies (16). Let us show that it satisfies (17). Suppose that $\left(x_{i}, a_{-i}\right) \in D^{\geq k},\left(y_{i}, a_{-i}\right) \in D^{\geq k},\left(y_{i}, b_{-i}\right) \in D^{\geq k},\left(x_{i}, b_{-i}\right) \in D^{<k}$ and $\left(z_{i}, a_{-i}\right) \in D^{<k}$, for some $x_{i}, y_{i}, z_{i} \in Y_{i}^{k}$ and some $a_{-i}, b_{-i} \in Y_{-i}^{k}$. Because $z_{i} \in Y_{i}^{k}$, we know that $\left(z_{i}, c_{-i}\right) \in D^{\geq k}$, for some $c_{-i} \in Y_{-i}^{k}$. Using $3 \mathrm{v}_{\text {-graded }}^{R}$, $\left(x_{i}, a_{-i}\right) \in D^{\geq k},\left(y_{i}, a_{-i}\right) \in D^{\geq k},\left(y_{i}, b_{-i}\right) \in D^{\geq k}$ and $\left(z_{i}, c_{-i}\right) \in D^{\geq k}$ imply $\left(x_{i}, b_{-i}\right) \in D^{\geq k}$ or $\left(z_{i}, a_{-i}\right) \in D^{\geq k}$, a contradiction.

Hence, using Proposition 21, we know that $\left\langle D^{\geq k}, D^{<k}\right\rangle$ has a representation $\left\langle\mathscr{F}^{k},\left\langle\mathscr{A}_{i}^{k}\right\rangle_{i \in N}\right\rangle$ in the noncompensatory sorting model. The uniqueness of this representation is detailed in Proposition 21.

The rest of the proof uses the following three observations.

\section{Observation 39}

Let $t \in R^{+}$and suppose that there is no attribute influent for $\left\langle D^{\geq t}, D^{<t}\right\rangle$. Let $\ell$ be the largest $k \in R^{+}$such that $k<t$ and there is at least one influent attribute for $\left\langle D^{\geq k}, D^{<k}\right\rangle$. Hence, for all $k \in R^{+}$such that $\ell<k \leq t$, we know that $D^{\geq k}=Y^{k}$. 
Let $\left\langle\mathscr{F}^{\ell},\left\langle\mathscr{A}_{i}^{\ell}\right\rangle_{i \in N}\right\rangle$ be any representation of $\left\langle D^{\geq \ell}, D^{<\ell}\right\rangle$ in the noncompensatory sorting model. For all $k \in R^{+}$such that $\ell<k \leq t$, we claim that taking:

$$
\text { - } \mathscr{A}_{i}^{k}=Y_{i}^{k} \cap \mathscr{A}_{i}^{\ell} \text {, for all } i \in N \text {, }
$$

- $\mathscr{F}^{k}=\mathscr{F}^{\ell}$,

gives a representation $\left\langle\mathscr{F}^{k},\left\langle\mathscr{A}_{i}^{k}\right\rangle_{i \in N}\right\rangle$ of $\left\langle D^{\geq k}, D^{<k}\right\rangle$ in the noncompensatory sorting model.

Indeed, we know that $D^{\geq k}=Y^{k}$. If $x \in D^{\geq k}=Y^{k}$, we have $x \in D^{\geq \ell}$, so that, by construction, $\left\{i \in N: x_{i} \in \mathscr{A}_{i}^{\ell}\right\} \in \mathscr{F}^{\ell}=\mathscr{F}^{k}$. Because $x \in Y^{k}$, we have $x_{i} \in Y_{i}^{k}$. Hence, $x_{i} \in \mathscr{A}_{i}^{\ell}$ implies $x_{i} \in \mathscr{A}_{i}^{k}=Y_{i}^{k} \cap \mathscr{A}_{i}^{\ell}$, so that $\left\{i \in N: x_{i} \in \mathscr{A}_{i}^{k}\right\} \in \mathscr{F}^{k}$. The converse is obvious since $D^{\geq k}=Y^{k}$.

Observe that for all $k \in R^{+}$such that $\ell<k \leq t$, the constraints $\mathscr{F}^{k} \subseteq$ $\mathscr{F}^{k-1}$ are obviously satisfied. Similarly, since $Y_{i}^{k} \subseteq Y_{i}^{k-1}$, we have that $\mathscr{A}_{i}^{k} \subseteq \mathscr{A}_{i}^{k-1}$.

Now let $k, k^{\prime} \in R^{+}$such that $\ell<k<k^{\prime}$ and suppose that $x_{i} \in \mathscr{A}_{i}^{k}$, $y_{i} \in \mathscr{U}_{i}^{k}=Y_{i}^{k} \backslash \mathscr{A}_{i}^{k}$. By construction, $x_{i} \in \mathscr{A}_{i}^{k}$ and $y_{i} \in \mathscr{U}_{i}^{k}$ imply that $\left(x_{i}, a_{-i}\right) \in D^{\geq \ell}$ and $\left(y_{i}, a_{-i}\right) \in D^{<\ell}$, for some $a_{-i} \in Y_{-i}^{\ell}$. Suppose now that $x_{i} \in \mathscr{V}_{i}^{k^{\prime}}$ and $y_{i} \notin \mathscr{V}_{i}^{k^{\prime}}$. By construction, we have that $\left(y_{i}, b_{-i}\right) \in D^{\geq k^{\prime}}$, for some $b_{-i} \in Y_{-i}^{k^{\prime}}$. Using linear ${ }_{i}^{R},\left(y_{i}, b_{-i}\right) \in D^{\geq k^{\prime}}$ and $\left(x_{i}, a_{-i}\right) \in D^{\geq \ell}$ imply either $\left(x_{i}, b_{-i}\right) \in D^{\geq k^{\prime}}$ or $\left(y_{i}, a_{-i}\right) \in D^{\geq \ell}$, a contradiction. Hence, the consistency condition (iii) holds with this construction.

\section{Observation 40}

Let $t \in R^{+}$and suppose that there is no attribute influent for $\left\langle D^{\geq t}, D^{<t}\right\rangle$. Suppose furthermore that for all $k \leq t$, all attributes are degenerate for $\left\langle D^{\geq k}, D^{<k}\right\rangle$. In such a case, taking:

- $\mathscr{A}_{i}^{k}=Y_{i}^{k}$, for all $i \in N$,

$$
\text { - } \mathscr{F}^{k}=2^{N} \text {, }
$$

obviously gives a representation of $\left\langle D^{\geq k}, D^{<k}\right\rangle$ in the noncompensatory sorting model, for all $k \leq t$. With such a construction, the constraints $\mathscr{F}^{k} \subseteq$ $\mathscr{F}^{k-1}$ are obviously satisfied, for all $k \leq t$. Similarly, since $Y_{i}^{k} \subseteq Y_{i}^{k-1}$, the constraints $\mathscr{A}_{i}^{k} \subseteq \mathscr{A}_{i}^{k-1}$ will always be satisfied, for all $k \leq t$. For all $k \leq t$, we have either $x_{i} \in \mathscr{A}_{i}^{k}$ or $x_{i} \in \mathscr{V}_{i}^{k}$, so that the consistency condition (iii) holds.

\section{Observation 41}

Let $s, t \in R^{+}$with $s<t$. Suppose that both $\left\langle D^{\geq s}, D^{<s}\right\rangle$ and $\left\langle D^{\geq t}, D^{<t}\right\rangle$ are partitions. Suppose furthermore that, for all $k \in R^{+}$such that $s<k<t$, all attributes are degenerate for $\left\langle D^{\geq k}, D^{<k}\right\rangle$. 
Let $\left\langle\mathscr{F}^{t},\left\langle\mathscr{A}_{i}^{t}\right\rangle_{i \in N}\right\rangle$ be a representation of $\left\langle D^{\geq t}, D^{<t}\right\rangle$ in the noncompensatory sorting model. If $i \in N$ is influent for $\left\langle D^{\geq t}, D^{<t}\right\rangle$, the set $\mathscr{A}_{i}^{t}$ is uniquely defined. If $i \in N$ is degenerate for $\left\langle D^{\geq t}, D^{<t}\right\rangle$, we know from Proposition 21 that we may take $\mathscr{A}_{i}^{t}$ to be an arbitrary subset of $Y_{i}^{t}$. Suppose that we have taken it as follows:

- if, for all $k \in R^{+}$such that $k>t$, attribute $i \in N$ is degenerate for $\left\langle D^{\geq k}, D^{<k}\right\rangle$, we take $\mathscr{A}_{i}^{t}=\varnothing$,

- otherwise, let $t^{+}$be the smallest $k \in R^{+}$such that $k>t$ and attribute $i \in N$ is influent for $\left\langle D^{\geq k}, D^{<k}\right\rangle$. In this case, we take $\mathscr{A}_{i}^{t}=\mathscr{A}_{i}^{t^{+}}$.

Observe that, in any case, we have $\mathscr{A}_{i}^{t} \subsetneq Y_{i}^{t}$.

Let $\left\langle\mathscr{F}^{s},\left\langle\mathscr{A}_{i}^{s}\right\rangle_{i \in N}\right\rangle$ be the representation of $\left\langle D^{\geq s}, D^{<s}\right\rangle$ in the noncompensatory sorting model derived from Proposition 21.

If $i \in N$ is degenerate for $\left\langle D^{\geq s}, D^{<s}\right\rangle$, we know that we may modify $\mathscr{A}_{i}^{s}$ taking it to be an arbitrary subset of $Y_{i}^{s}$. In this case, we take $\mathscr{A}_{i}^{s}=\mathscr{A}_{i}^{t}$, so that we will have $\mathscr{A}_{i}^{t} \subseteq \mathscr{A}_{i}^{s}$.

If $i \in N$ is influent for $\left\langle D^{\geq s}, D^{<s}\right\rangle$, we have $\varnothing \subsetneq \mathscr{A}_{i}^{s} \subsetneq Y_{i}^{s}$. If attribute $i \in N$ is degenerate for all $k \in R^{+}$such that $k \geq t$, we have $\mathscr{A}_{i}^{t}=\varnothing$, so that $\mathscr{A}_{i}^{t} \subseteq \mathscr{A}_{i}^{s}$. Otherwise, let $t^{+}$be the smallest $k \in R^{+}$such that $k \geq t$ and attribute $i \in N$ is influent for $\left\langle D^{\geq k}, D^{<k}\right\rangle$. We have $\mathscr{A}_{i}^{t}=\mathscr{A}_{i}^{t^{+}}$. Let us show that $\mathscr{A}_{i}^{t^{+}} \subseteq \mathscr{A}_{i}^{s}$.

Suppose that $x_{i} \in \mathscr{A}_{i}^{t^{+}}$and $x_{i} \notin \mathscr{A}_{i}^{s}$. Since $i \in N$ is influent for the partition $\left\langle D^{\geq t^{+}}, D^{<t^{+}}\right\rangle$and $x_{i} \in \mathscr{A}_{i}^{t^{+}}$, we know that $\left(x_{i}, a_{-i}\right) \in D^{\geq t^{+}}$and $\left(y_{i}, a_{-i}\right) \notin D^{\geq t^{+}}$, for some $y_{i} \in Y_{i}^{t^{+}}$and some $a_{-i} \in Y_{-i}^{t^{+}}$. Because, by construction, $y_{i} \in Y_{i}^{t^{+}}$, we know that $\left(y_{i}, c_{-i}\right) \in D^{\geq t^{+}}$, for some $c_{-i} \in Y_{-i}^{t^{+}}$. Similarly, since $i \in N$ is influent for $\left\langle D^{\geq s}, D^{<s}\right\rangle$ and $x_{i} \notin \mathscr{A}_{i}^{s}$, we know that $\left(z_{i}, b_{-i}\right) \in D^{\geq s}$ and $\left(x_{i}, b_{-i}\right) \notin D^{\geq s}$, for some $z_{i} \in Y_{i}^{s}$ and some $b_{-i} \in Y_{-i}^{s}$.

Using linear ${ }_{i}^{R},\left(x_{i}, a_{-i}\right) \in D^{\geq t^{+}},\left(z_{i}, b_{-i}\right) \in D^{\geq s}$ and $\left(x_{i}, b_{-i}\right) \notin D^{\geq s}$ imply $\left(z_{i}, a_{-i}\right) \in D^{\geq t^{+}}$. Using $3 v_{-g r a d e d}^{R},\left(x_{i}, a_{-i}\right) \in D^{\geq t^{+}},\left(z_{i}, a_{-i}\right) \in D^{\geq t^{+}}$, $\left(z_{i}, b_{-i}\right) \in D^{\geq s}$ and $\left(y_{i}, c_{-i}\right) \in D^{\geq t^{+}}$imply $\left(x_{i}, b_{-i}\right) \in D^{\geq s}$ or $\left(y_{i}, a_{-i}\right) \in D^{\geq t^{+}}$, a contradiction. Hence we have $\mathscr{A}_{i}^{t^{+}} \subseteq \mathscr{A}_{i}^{s}$.

Let us now show that $\mathscr{F}^{t} \subseteq \mathscr{F}^{s}$. Because $\mathscr{A}_{i}^{t}$ is always a strict subset of $Y_{i}^{t}$, we have $I \in \mathscr{F}^{t}$ whenever there is some $x \in D^{\geq t}$ such that $A^{t}(x) \subseteq I$. Similarly, since $\mathscr{A}_{i}^{s}$ is always a strict subset of $Y_{i}^{s}$, we have $I \in \mathscr{F}^{s}$ whenever there is some $x \in D^{\geq s}$ such that $A^{s}(x) \subseteq I$.

From now, the proof that $\mathscr{F}^{t} \subseteq \mathscr{F}^{s}$ is identical to that of Theorem 22 . Indeed, we know that $I \in \mathscr{F}^{t}$ whenever there is some $x \in D^{\geq t}$ such that $A^{t}(x) \subseteq I$. Take any such alternative $x \in D^{\geq t}$.

Starting with $x$, let us build an alternative $x^{\prime} \in Y^{t} \subseteq Y^{s}$ as follows. For all $i \in N$ such that $x_{i} \in \mathscr{A}_{i}^{t}$, let $x_{i}^{\prime}=x_{i}$. Because $\mathscr{A}_{i}^{t} \subseteq \mathscr{A}_{i}^{s}$, we know that 
on these attributes $x_{i}^{\prime} \in \mathscr{A}_{i}^{s}$. For all $i \in N$ such that $x_{i} \notin \mathscr{A}_{i}^{t}$, we consider two cases.

1. Suppose that $i \in N$ is influent for $\left\langle D^{\geq s}, D^{<s}\right\rangle$. By construction, there is a $z_{i} \in Y_{i}^{s}$ such that $z_{i} \notin \mathscr{A}_{i}^{s}$. In this case, let $x_{i}^{\prime}=z_{i}$. By construction, we know that $x_{i}^{\prime} \notin \mathscr{A}_{i}^{s}$ on these attributes.

2. Suppose that $i \in N$ is not influent for $\left\langle D^{\geq s}, D^{<s}\right\rangle$. By construction, we have taken $\mathscr{A}_{i}^{s}$ to be equal to $\mathscr{A}_{i}^{t}$. In this case, take $x_{i}^{\prime}$ equal to $x_{i}$. Therefore $x_{i}^{\prime} \notin \mathscr{A}_{i}^{s}$ on these attributes.

By construction, we have $A^{t}(x)=A^{t}\left(x^{\prime}\right)=A^{s}\left(x^{\prime}\right)=I$. Because $A^{t}(x)=$ $A^{t}\left(x^{\prime}\right)$ and $x \in D^{\geq t}$, we know that $x^{\prime} \in D^{\geq t}$ so that $x^{\prime} \in D^{\geq s}$. We have $x^{\prime} \in D^{\geq s}$ and $A^{s}\left(x^{\prime}\right) \subseteq I$. Hence, it must be true that $I \in \mathscr{F}^{s}$, so that we have proved that $\mathscr{F}^{t} \subseteq \mathscr{F}^{s}$.

Let us now show that the consistency condition (iii) holds. Suppose that $x_{i} \in \mathscr{A}_{i}^{s}, y_{i} \in \mathscr{U}_{i}^{s}$ and $x_{i} \in \mathscr{V}_{i}^{t}$. We have to show that $y_{i} \in \mathscr{V}_{i}^{t}$.

Suppose first that attribute $i \in N$ is influent for $\left\langle D^{\geq s}, D^{<s}\right\rangle$. Because $x_{i} \in \mathscr{A}_{i}^{s}, y_{i} \in \mathscr{U}_{i}^{s}$, we have $\left(x_{i}, a_{-i}\right) \in D^{\geq s}$ and $\left(y_{i}, a_{-i}\right) \notin D^{\geq s}$, for some $a_{-i} \in Y_{-i}^{s}$. In contradiction with the thesis, suppose that $\left(y_{i}, b_{-i}\right) \in D^{\geq t}$, for some $b_{-i} \in Y_{-i}^{t}$. Using linear ${ }_{i}^{R},\left(x_{i}, a_{-i}\right) \in D^{\geq s}$ and $\left(y_{i}, b_{-i}\right) \in D^{\geq t}$ imply $\left(y_{i}, a_{-i}\right) \in D^{\geq s}$ or $\left(x_{i}, b_{-i}\right) \in D^{\geq t}$. This is contradictory since we know that $\left(y_{i}, a_{-i}\right) \notin D^{\geq s}$ and $x_{i} \in \mathscr{V}_{i}^{t}$.

Suppose now that attribute $i \in N$ is not influent for $\left\langle D^{\geq s}, D^{<s}\right\rangle$. In this case, we have $\mathscr{A}_{i}^{s}=\mathscr{A}_{i}^{t}$ so that it is impossible to have $x_{i} \in \mathscr{A}_{i}^{s}$ and $x_{i} \in \mathscr{V}_{i}^{t}$.

Using the above observations, we complete the proof. Let $t^{1} \in R^{+}$be the largest $k \in R^{+}$such that $\left\langle D^{\geq k}, D^{<k}\right\rangle$ is a partition. Let $\left\langle\mathscr{G}^{t^{1}},\left\langle\mathscr{B}_{i}^{t^{1}}\right\rangle_{i \in N}\right\rangle$ be the representation of $\left\langle D^{\geq t^{1}}, D^{<t^{1}}\right\rangle$ derived from Proposition 21. We take $\mathscr{F}^{t^{1}}=\mathscr{G}^{t^{1}}$ and $\mathscr{A}_{i}^{t^{1}}=\mathscr{B}_{i}^{t^{1}}$.

For all $k \in R^{+}$such that $k>t^{1}$, we define $\mathscr{A}_{i}^{k}$ and $\mathscr{F}^{k}$ using Observation 39. If, for all $k \in R^{+}$such that $k<t^{1}$, there is no influent attribute for $\left\langle D^{\geq k}, D^{<k}\right\rangle$, we define $\mathscr{A}_{i}^{k}$ and $\mathscr{F}^{k}$ using Observation 40.

Otherwise, let $t^{2}$ be the largest $k \in R^{+}$such that $k<t^{1}$ and there is at least one influent attribute for $\left\langle D^{\geq k}, D^{<k}\right\rangle$. In this case, we define $\mathscr{A}_{i}^{t^{2}}$ and $\mathscr{F}^{t^{2}}$ using Observation 41 and for all $k \in R^{+}$such that $t^{2}<k<t^{1}$, we define $\mathscr{A}_{i}^{k}$ and $\mathscr{F}^{k}$ using Observation 39.

Iterating the above process leads to define a representation of $\left\langle C^{k}\right\rangle_{k \in R}$ in the noncompensatory sorting model with veto.

The construction of a representation in the noncompensatory sorting model with veto is illustrated below. 


\section{Example 42}

Suppose that $n=3, X_{1}=X_{2}=X_{3}=\{8,9,10,11\}$. We consider a threefold partition $\left\langle C^{1}, C^{2}, C^{3}\right\rangle$. Let $C^{3}=\{(10,9,10),(10,9,11),(10,10,10),(10,10$, $11),(10,11,10),(10,11,11),(11,9,10),(11,9,11),(11,10,10),(11,10,11)$, $(11,11,10),(11,11,11)\}, C^{2}=\{(8,10,9),(8,10,10),(8,10,11),(8,11,9)$, $(8,11,10),(8,11,11),(9,10,9),(9,10,10),(9,10,11),(9,11,9),(9,11,10)$, $(9,11,11),(10,9,9),(10,10,8),(10,10,9),(10,11,8),(10,11,9),(11,9,9)$, $(11,10,8),(11,10,9),(11,11,8),(11,11,9)\}$ and $C^{1}=X \backslash\left[C^{3} \cup C^{2}\right]$.

This partition can be obtained with the pessimistic version of ELECTRE TRI nested relations with $(10,10,10)$ as the limiting profile between $C^{3}$ and $C^{2}$ and $(10,10,9)$ as the limiting profile between $C^{2}$ and $C^{1}, S_{i}=\geq$ for all $i \in N, w_{1}=w_{3}=0.4, w_{2}=0.2, \lambda^{3}=0.7, \lambda^{2}=0.5, V_{1}=\varnothing$ and $V_{i}=\{(10,8),(11,8)\}$, for $i \in\{2,3\}$. This shows that this partition is $R$ linear and $R$-3v-graded.

We have:

- $\mathscr{V}_{1}^{3}=\mathscr{V}_{3}^{3}=\{8,9\}, \mathscr{V}_{2}^{3}=\{8\}$,

- $\mathscr{V}_{1}^{2}=\mathscr{V}_{3}^{2}=\varnothing, \mathscr{V}_{2}^{2}=\{8\}$.

For the twofold partition $\left\langle D^{\geq 3}, D^{<3}\right\rangle$ on $Y^{3}=\{10,11\} \times\{9,10,11\} \times$ $\{10,11\}$, all attributes are degenerate. All attributes are influent for the twofold partition $\left\langle D^{\geq 2}, D^{<2}\right\rangle$ on $Y^{2}=\{8,9,10,11\} \times\{9,10,11\} \times\{8,9,10,11\}$.

Let $\left\langle\mathscr{G}^{2},\left\langle\mathscr{B}_{1}^{2}, \mathscr{B}_{2}^{2}, \mathscr{B}_{3}^{2}\right\rangle\right\rangle$ be the unique representation of $\left\langle D^{\geq 2}, D^{<2}\right\rangle$ induced on $Y^{2}$ by $\left\langle C^{\geq 2}, C^{<2}\right\rangle$ derived from Proposition 21. We have $\mathscr{B}_{1}^{2}=$ $\{10,11\}, \mathscr{B}_{2}^{2}=\{10,11\}, \mathscr{B}_{3}^{2}=\{9,10,11\}$ and $\mathscr{G}^{2}=\{\{1,2\},\{1,3\},\{2,3\},\{1,2,3\}\}$. We take $\mathscr{A}_{i}^{2}=\mathscr{B}_{i}^{2}$, for all $i \in N$ and $\mathscr{F}^{2}=\mathscr{G}^{2}$. As in the above proof, we take $\mathscr{A}_{i}^{3}=Y_{i}^{3} \cap \mathscr{A}_{i}^{2}$, for all $i \in N$ and $\mathscr{F}^{3}=\mathscr{F}^{2}$.

It can easily be checked that $\left\langle\mathscr{F}^{k},\left\langle\mathscr{A}_{i}^{k}, \mathscr{V}_{i}^{k}\right\rangle_{i \in N}\right\rangle$ is a representation of $\langle\mathscr{A}, \mathscr{U}\rangle$ in the noncompensatory sorting model with veto.

\subsection{Independence and uniqueness}

In view of the complexity of the noncompensatory sorting model with veto, we do not pursue here a detailed analysis of particular cases of the noncompensatory sorting model with veto as was done in Section 5.8. Such an analysis is likely to be quite cumbersome. We simply analyze below the independence of the conditions used in Theorem 38 and the uniqueness of the representation in the noncompensatory sorting model with veto. 


\subsubsection{Independence of conditions}

Example 26 above gives a partition that is $R$-2-graded and that satisfies linear $_{i}^{R}$ on all but one attribute. Since $R$-2-gradedness implies $R$-3v-gradedness, this gives an example showing that, in Theorem 38, no condition $\operatorname{linear}_{i}^{R}$ is redundant.

The following example shows that a partition may be $R$-linear and may satisfy $3 \mathrm{v}$-graded ${ }_{i}^{R}$ on all but one attribute.

\section{Example 43}

Let $n=3, X=\left\{x_{1}, y_{1}, z_{1}\right\} \times\left\{x_{2}, y_{2}, z_{2}\right\} \times\left\{x_{3}, y_{3}, z_{3}\right\}$ and $r=4$. Let $C^{4}=\left\{\left(x_{1}, x_{2}, x_{3}\right),\left(y_{1}, x_{2}, x_{3}\right),\left(z_{1}, x_{2}, x_{3}\right),\left(x_{1}, y_{2}, x_{3}\right),\left(y_{1}, y_{2}, x_{3}\right),\left(z_{1}, y_{2}, x_{3}\right)\right.$, $\left.\left(x_{1}, x_{2}, y_{3}\right),\left(y_{1}, x_{2}, y_{3}\right),\left(z_{1}, x_{2}, y_{3}\right),\left(x_{1}, y_{2}, y_{3}\right),\left(y_{1}, y_{2}, y_{3}\right)\right\}, C^{3}=\left\{\left(z_{1}, y_{2}, y_{3}\right)\right\}$, $C^{2}=\left\{\left(x_{1}, x_{2}, z_{3}\right),\left(y_{1}, x_{2}, z_{3}\right),\left(x_{1}, y_{2}, z_{3}\right),\left(y_{1}, y_{2}, z_{3}\right),\left(x_{1}, z_{2}, x_{3}\right),\left(y_{1}, z_{2}, x_{3}\right)\right.$, $\left.\left(x_{1}, z_{2}, y_{3}\right),\left(y_{1}, z_{2}, y_{3}\right),\left(y_{1}, z_{2}, z_{3}\right)\right\}$ and $C^{1}=\left\{\left(x_{1}, z_{2}, z_{3}\right),\left(z_{1}, z_{2}, z_{3}\right),\left(z_{1}, y_{2}, z_{3}\right)\right.$, $\left.\left(z_{1}, z_{2}, y_{3}\right),\left(z_{1}, x_{2}, z_{3}\right),\left(z_{1}, z_{2}, x_{3}\right)\right\}$.

We have $y_{1} \succ_{1}^{R} x_{1} \succ_{1}^{R} z_{1}, x_{2} \succ_{2}^{R} y_{2} \succ_{2}^{R} z_{2}$ and $x_{3} \succ_{3}^{R} y_{3} \succ_{3}^{R} z_{3}$. This shows that the partition is $R$-linear.

Condition $3 v$-graded ${ }_{1}^{R}$ is violated since $\left(x_{1}, y_{2}, y_{3}\right) \in C^{\geq 4},\left(y_{1}, y_{2}, y_{3}\right) \in$ $C^{\geq 4},\left(y_{1}, z_{2}, z_{3}\right) \in C^{\geq 2}$ and $\left(z_{1}, x_{2}, x_{3}\right) \in C^{\geq 4}$, while $\left(x_{1}, z_{2}, z_{3}\right) \notin C^{\geq 2}$ and $\left(z_{1}, y_{2}, y_{3}\right) \notin C^{\geq 4}$.

We have $x_{2} \succ_{2}^{4} y_{2} \succ_{2}^{4} z_{2},\left[x_{2} \sim_{2}^{3} y_{2}\right] \succ_{2}^{3} z_{2}$ and $\left[x_{2} \sim_{2}^{2} y_{2}\right] \succ_{2}^{2} z_{2}$. We never have $\left(\alpha_{1}, z_{2}, \alpha_{3}\right) \in C^{\geq 4}$. Using Lemma 37 , this shows that $3 \mathrm{v}$-graded ${ }_{2}^{R}$ holds. Similarly, it is easy to check that $3 \mathrm{v}$-graded ${ }_{3}^{R}$ holds.

Hence, we have shown that none of the conditions used in Theorem 38 is redundant. Note that, in Example 43, the weakening of condition $3 \mathrm{v}$-graded ${ }_{i}^{R}$ obtained requiring $3 \mathrm{v}-\operatorname{graded}_{i}^{R}$ only when $k=\ell$ is satisfied, for all $i \in N$.

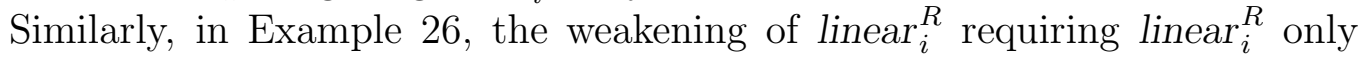
when $\ell=k$ is satisfied, for all $i \in N$. Hence, our two conditions may not be weakened in this way.

\subsubsection{Uniqueness}

Let $\langle\mathscr{A}, \mathscr{U}\rangle$ be a twofold partition of $X$. Define $Z_{i}=\left\{x_{i} \in X_{i}:\left(x_{i}, a_{-i}\right) \in\right.$ $\mathscr{U}$, for all $\left.a_{-i} \in X_{-i}\right\}$ and $Y_{i}=X_{i} \backslash Z_{i}$. Let $Y=\prod_{i=1}^{n} Y_{i}$ and define $\mathscr{A}^{\prime}=$ $\mathscr{A} \cap Y$ and $\mathscr{U}^{\prime}=\mathscr{U} \cap Y$. We show in Bouyssou and Marchant (2005) that the representation of $\langle\mathscr{A}, \mathscr{U}\rangle$ is unique if and only if all attributes $i \in N$ are influent for $\left\langle\mathscr{A}^{\prime}, \mathscr{U}^{\prime}\right\rangle$.

As was the case for the noncompensatory sorting model, the additional constraints brought by the noncompensatory sorting model with veto with more than two categories are such that this sufficient condition is no longer 
necessary. Since the noncompensatory sorting model is a particular case of the noncompensatory sorting model with veto, Example 28 illustrates this possibility. The uniqueness of the representation in the noncompensatory sorting model with veto can be analyzed using the same lines as in Section 5.7. Since the details are cumbersome and not informative, we do not develop this point here. It should nevertheless be clear that as soon as some attribute is degenerate for a twofold partition $\left\langle D^{\geq k}, D^{<k}\right\rangle$, the uniqueness of the representation in the noncompensatory sorting model with veto will be quite unlikely.

Let us finally observe that, as in Remark 20, it is not difficult to use the above results to show that a partition that can be obtained with ELECTRE TRI using profiles that are outside the set $X$ can always be obtained with ELECTRE TRI using profiles that belong to $X$ (this is obvious if the sets $\mathscr{A}_{i}^{r} \subseteq \mathscr{A}_{i}^{r-1} \subseteq \cdots \subseteq \mathscr{A}_{i}^{k} \subseteq \cdots \subseteq \mathscr{A}_{i}^{2}$ and $\mathscr{V}_{i}^{r} \supseteq \mathscr{V}_{i}^{r-1} \supseteq \cdots \supseteq \mathscr{V}_{i}^{2}$ are all distinct and nonempty. It is simple to extend this conclusion when some of these sets are equal or empty).

\section{Conclusion}

This paper has provided a characterization of the noncompensatory sorting model with and without veto, extending the results presented in Bouyssou and Marchant (2005) to an arbitrary (finite) number of ordered categories. This characterization was performed within a general framework for sorting models studied in Greco et al. (2001b) and Słowiński et al. (2002) (see also Goldstein, 1991, for the case of two and three categories) that obtains for $R$-linear partitions. This characterization shows that the main distinctive characteristic of these models lies in the rather poor information they use on each attribute. This feature was captured using either $R$-2-gradedness (for the case without veto) or by $R$-3v-gradedness (for the case with veto). These conditions are central for the ELECTRE TRI sorting model. Hence, the reasonableness of this model is clearly linked with the reasonableness of these two conditions.

We refer to the discussion section of Bouyssou and Marchant (2005) for a detailed analysis of the theoretical and practical implications of our results. Let us briefly mention here, some important ones:

- Our theoretical analysis shows that the two versions of the ELECTRE TRI method are rather different. Only the pessimistic version fits into the framework of the noncompensatory sorting model with veto. This might be interpreted as an indication of the fact that our definition of the noncompensatory sorting model with veto is too restrictive. We 
rather think that this is linked to the fact that the optimistic version of ELECTRE TRI is not primarily based on the outranking relation $S$ but on its asymmetric part and in rather an undirect way.

- Our analysis has lead us to suggest a variant of the original ELECTRE TRI method that uses a sequence of nested relations. Because this modification is minor and nevertheless allows to increase the descriptive power of the technique, i.e., its ability to represent a given partition, we believe that it would be worthwhile to consider it in real-world applications.

- Our results show that the representation of a partition in the noncompensatory sorting model with veto is not likely to be unique. This has clearly an impact on the way to approach methods trying to infer the parameters of an ELECTRE TRI model from assignment examples (i.e., from a partition defined on a subset of $X$ ) using mathematical programming techniques (see Dias and Mousseau, 2006; Dias et al., 2002; Mousseau et al., 2001a; Mousseau and Słowiński, 1998; Ngo The and Mousseau, 2002). Given this non-uniqueness, a particular attention should be given to the derivation of robust recommendations on the basis of such methods, i.e., recommendations that remains valid for all possible values of the parameters that are compatible with the assignment examples.

The analysis proposed in this paper can, and should, be extended in several directions. It would be interesting to use the rich framework offered by model $(D 1)$ to tackle the case of other sorting methods. The authors have started a research on the additive specialization of model $(D 1)$ that is at the heart of the famous UTADIS technique (see, e.g, Jacquet-Lagrèze, 1995; Zopounidis and Doumpos, 2000a,b, 2001). Second, the axiomatic analysis undertaken here would clearly call for experimental investigations of the reasonableness of the conditions exhibited. As already stressed in Goldstein (1991), experimental violations of $R$-linearity would have rather important consequences. This might render the analysis in Greco et al. (2001b) and Słowiński et al. (2002) studying models tolerating violations of $R$-linearity all the more important.

Summarizing, it seems that sorting models offer a widely open field for foundational research in the area of MCDM and that the general framework for sorting models studied in Greco et al. (2001b) and Słowiński et al. (2002) seems to be quite convenient to guide such an investigation. 


\section{References}

Andenmatten, A. (1995). Évaluation du risque de défaillance des émetteurs d'obligations, une approche par l'aide multicritère à la décision. Presses Polytechniques et Universitaires Romandes, Lausanne.

Arondel, C. and Girardin, P. (2000). Sorting cropping systems on the basis of their impact on groundwater quality. European Journal of Operational Research, 127(3):467-482.

Bouyssou, D. and Marchant, Th. (2005). An axiomatic approach to noncompensatory sorting methods in MCDM, I: The case of two categories. Working paper, LAMSADE, Université Paris Dauphine, available at www.lamsade.dauphine. $\mathrm{fr} / \sim$ bouyssou/.

Bouyssou, D. and Pirlot, M. (1999). Conjoint measurement without additivity and transitivity. In Meskens, N. and Roubens, M., editors, Advances in Decision Analysis, pages 13-29. Kluwer, Dordrecht.

Bouyssou, D. and Pirlot, M. (2002a). A characterization of strict concordance relations. In Bouyssou, D., Jacquet-Lagrèze, É., Perny, P., Słowiński, R., Vanderpooten, D., and Vincke, Ph., editors, Aiding Decisions with Multiple Criteria: Essays in Honour of Bernard Roy, pages 121-145. Kluwer, Dordrecht.

Bouyssou, D. and Pirlot, M. (2002b). Nontransitive decomposable conjoint measurement. Journal of Mathematical Psychology, 46:677-703.

Bouyssou, D. and Pirlot, M. (2004a). 'Additive difference' models without additivity and subtractivity. Journal of Mathematical Psychology, 48(4):263-291.

Bouyssou, D. and Pirlot, M. (2004b). Preferences for multiattributed alternatives: Traces, dominance, and numerical representations. Journal of Mathematical Psychology, 48(3):167-185.

Bouyssou, D. and Pirlot, M. (2005). A characterization of concordance relations. European Journal of Operational Research, 167(2):427-443.

Dias, L. C. and Clímaco, J. (2000). ELECTRE TRI for groups with imprecise information on parameter values. Group Decision and Negotiation, 9:355-377.

Dias, L. C. and Mousseau, V. (2006). Inferring ELECTRE's veto-related parameters from outranking examples. European Journal of Operational Research, 170(1):172-191.

Dias, L. C., Mousseau, V., Figueira, J., and Clímaco, J. (2002). An aggregation / disaggregation approach to obtain robust conclusions with ELECTRE TRI. European Journal of Operational Research, 138:332-48.

Fishburn, P. C. (1970). Utility theory for decision-making. Wiley, New York.

Georgopoulou, E., Sarafidis, Y., Mirasgedis, S., Zaimi, S., and Lalas, D. (2003). A multiple criteria decision-aid approach in defining national priorities for greenhouse gases emissions reduction in the energy sector. European Journal of Operational Research, 146(1):199-215. 
Goldstein, W. M. (1991). Decomposable threshold models. Journal of Mathematical Psychology, 35:64-79.

Greco, S., Matarazzo, B., and Słowiński, R. (1999a). Rough approximation of a preference relation by dominance relations. European Journal of Operational Research, 117:63-83.

Greco, S., Matarazzo, B., and Słowiński, R. (1999b). The use of rough sets and fuzzy sets in MCDM. In Gal, T., Hanne, T., and Stewart, T., editors, Multicriteria decision making, Advances in MCDM models, algorithms, theory and applications, pages 14.1-14.59, Dordrecht. Kluwer.

Greco, S., Matarazzo, B., and Słowiński, R. (2001a). Axiomatic basis of noncompensatory preferences. Communication to FUR X, 30 May-2 June, Torino, Italy.

Greco, S., Matarazzo, B., and Słowiński, R. (2001b). Conjoint measurement and rough set approach for multicriteria sorting problems in presence of ordinal criteria. In Colorni, A., Paruccini, M., and Roy, B., editors, A-MCD-A, Aide Mulcritère à la Décision / Multiple Criteria Decision Aid, pages 117-144. European Commission, Joint Research Centre, Luxembourg.

Greco, S., Matarazzo, B., and Słowiński, R. (2001c). Rough sets theory for multicriteria decision analysis. European Journal of Operational Research, 129:1-7.

Greco, S., Matarazzo, B., and Słowiński, R. (2002a). Multicriteria classification. In Klogsen, W. and Zytkow, J., editors, Handbook of data mining and knowledge discovery, pages 318-328. Oxford University Press, Oxford.

Greco, S., Matarazzo, B., and Słowiński, R. (2002b). Rough sets methodology for sorting problems in presence of multiple attributes and criteria. European Journal of Operational Research, 138:247-59.

Greco, S., Matarazzo, B., and Słowiński, R. (2005). Decision rule approach. In Figueira, J., Greco, S., and Ehrgott, M., editors, Multiple Criteria Decision Analysis: State of the Art Surveys, pages 507-562. Springer Verlag, Boston, Dordrecht, London.

Jacquet-Lagrèze, É. (1995). An application of the UTA discriminant model for the evaluation of R\&D projects. In Pardalos, P., Siskos, Y., and Zopounidis, C., editors, Advances in Multicriteria Analysis, pages 203-211. Kluwer, Dordrecht.

Krantz, D. H., Luce, R. D., Suppes, P., and Tversky, A. (1971). Foundations of measurement, vol. 1: Additive and polynomial representations. Academic Press, New York.

Lourenco, R. P. and Costa, J. P. (2004). Using ELECTRE TRI outranking method to sort MOMILP non dominated solutions. European Journal of Operational Research, 153(2):271-289.

Marichal, J.-L. (2000). On Sugeno integrals as an aggregation function. Fuzzy Sets and Systems, 114.

Moussa, N. (2001). Aide multicritère à l'évaluation qualitative par inférence de 
modèles de tri ordinal sur une hiérarchie de critères. Thèse de doctorat, Université Paris-Dauphine.

Mousseau, V., Figueira, J., and Naux, J.-Ph. (2001a). Using assignment examples to infer weights for ELECTRE TRI method: Some experimental results. European Journal of Operational Research, 130:263-275.

Mousseau, V., Roy, B., and Sommerlatt, I. (2000a). Elaboration d'un outil d'aide à la décision en vue de l'évolution de la tarification des transports publics en Ile de France. Journal of Decision Systems, 9(2):289-315.

Mousseau, V., Roy, B., and Sommerlatt, I. (2001b). Development of a decision aiding tool for the evolution of public transport ticket pricing in the Paris region. In A. Colorni, M. P. and Roy, B., editors, A-MCD-A Aide Multicritère à la Décision - Multiple Criteria Decision Aiding, pages 213-230. European Commission, Joint Research Centre, Luxembourg.

Mousseau, V. and Słowiński, R. (1998). Inferring an ELECTRE TRI model from assignment examples. Journal of Global Optimization, 12:157-174.

Mousseau, V., Słowiński, R., and Zielniewicz, P. (2000b). A user-oriented implementation of the ELECTRE TRI method integrating preference elicitation support. Computers \& Operations Research, 27:757-777.

Ngo The, A. and Mousseau, V. (2002). Using assignment examples to infer category limits for the ELECTRE TRI method. Journal of Multi-Criteria Decision Analysis, 11(1):29-43.

Roy, B. (2002). Présentation et interprétation de la méthode ELECTRE TRI pour affecter des zones dans des catégories de risque. Document du LAMSADE 124, Université de Paris Dauphine. 25 pages.

Roy, B. and Bouyssou, D. (1993). Aide multicritère à la décision : méthodes et cas. Economica, Paris.

Słowiński, R., Greco, S., and Matarazzo, B. (2002). Axiomatization of utility, outranking and decision-rule preference models for multiple-criteria classification problems under partial inconsistency with the dominance principle. Control and Cybernetics, 31(4):1005-1035.

Tervonen, T., Almeida-Dias, J., Figueira, J., Lahdelma, R., and Salminen, P. (2005). SMAA-TRI: A parameter stability analysis method for ELECTRE TRI. Research Report 6, INESC - Coimbra.

Wei, Y. (1992). Aide multicritère à la décision dans le cadre de la problématique du tri : concepts, méthodes et applications. Thèse de doctorat, Université Paris Dauphine, Paris.

Zopounidis, C. and Doumpos, M. (2000a). Intelligent decision aiding systems based on multiple criteria for financial engineering. Kluwer, Dordrecht.

Zopounidis, C. and Doumpos, M. (2000b). PREFDIS: A multicriteria decision support system for sorting decision problems. Computers $\&$ Operations Research, 27(7-8):779-797.

Zopounidis, C. and Doumpos, M. (2001). A preference disaggregation decision 
support system for financial classification problems. European Journal of Operational Research, 130(2):402-413.

Zopounidis, C. and Doumpos, M. (2002). Multicriteria classification and sorting methods: A literature review. European Journal of Operational Research, 138:229-246. 